BNWL-994 UC-25

\title{
PROMETHIUM-147 RADIOISOTOPE APPLICATION PROGRAM AMSA HEAT SOURCE FINAL REPORT
}

March 1969

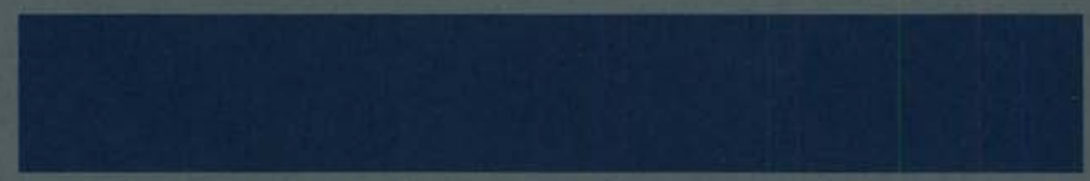

AEC RESEARCH \& DEVELOPMENT REPORT

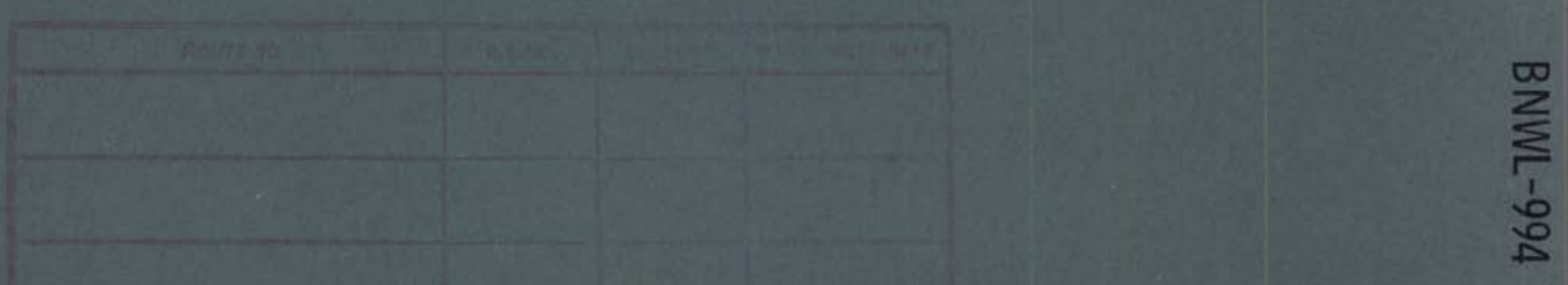




\section{LEGAL NOTICE}

This report was prepared as an account of Government sponsored wark. Neither the United States; nor the Commission, nor any person acting on behalf of the Commission:

A. Makes any warranty or representation, expressed or implied, with respect to the accuracy, completeness, or usefulness of the information contained in this report, or that the use of any information, apparatus, method, or process disclosed in this report may not infringe privately owned rights; or

B. Assumes any liabilities with respect to the use of, or for damages resulting from the use of any information, apparatus, method, or process disclosed in this report.

As used in the above, "person acting on behalf of the Commission" includes any employee or contractor of the Commission, or employee of such contractor, to the extent that such employee or contractor of the Commission, or employee of such contractor prepares, disseminates, or provides access to, any information pursuant to his employment or contract with the Commission, or his employment with such contractor.

\section{PACIFIC NORTHWEST LABORATORY \\ RICHLAND, WASHINGTON \\ operated by \\ BATTELLE MEMORIAL INSTITUTE}

for the

UNITED STATES ATOMIC ENERGY COMMISSION UNDER CONTRACT AT(45-1)-1830 
BNWL- 994

UC-25, Meta1s, Ceramics, and Materials

\section{PROMETHIUM-147 RADIOISOTOPE APPLICATION PROGRAM AMSA HEAT SOURCE \\ FINAL REPORT \\ By}

N. C. Davis and D. W. Brite

Fuels and Materials Department Chemistry and Metallurgy Division

March 1969

mas

BATTELLE MEMORIAL INSTITUTE

PACIFIC NORTHWEST LABORATORY

RICHLAND, WASHINGTON 99352 
BNWL -994

Printed in the United States of America Available from

Clearinghouse for Federal Scientific and Technical Information National Bureau of Standards, U.S. Department of Commerce Springfield, Virginia 22151

Price: Printed Copy $\$ 3.00$; Microfiche $\$ 0.65$ 


\section{PROMETHIUM-147 RADIOISOTOPE APPLICATION PROGRAM AMSA HEAT SOURCE \\ FINAL REPORT}

N. C. Davis and D. W. Brite

\section{ABSTRACT}

A promethium-147 fueled $60 \mathrm{~W}$ thermal source was fabricated for the Air Force for use in heating a navigation device for the Advanced Manned Strategic Aircraft (AMSA) Program. A high strength impact resistant capsule was designed, and a capsule fabrication process was developed. A series of dummy $\left(\mathrm{Sm}_{2} \mathrm{O}_{3}\right.$ stand-in for $\left.{ }^{147} \mathrm{Pm}_{2} \mathrm{O}_{3}\right)$ capsules were fabricated, impact tested, and destructively evaluated to demonstrate process quality and to determine impact strength. Process and quality control inspection procedures were established and the ${ }^{147} \mathrm{Pm}_{2} \mathrm{O}_{3}$ fueled capsule was produced and delivered to the Air Force for laboratory testing and flight demonstration of AMSA navigational equipment. 


\section{TABLE OF CONTENTS}

\begin{tabular}{|c|c|c|c|c|c|c|c|c|c|c|c|}
\hline LIST OF FIGURES . & - & - & - & - & - & • & - & & & & vii \\
\hline LIST OF TABLES & - & - & - & • & - & - & - & 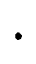 & 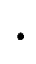 & 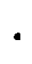 & ix \\
\hline INTRODUCT ION . & - & $\cdot$ & - & - & - & $\cdot$ & • & . & 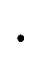 & - & 1 \\
\hline SUMMARY • • & - & - & - & - & - & - & - & ${ }^{\circ}$ & ${ }^{\circ}$ & $\bullet$ & 3 \\
\hline Capsule Developmen & & • & - & - & - & - & - & . & 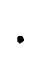 & - & 3 \\
\hline Capsule Qualificat & tion & - & • & - & - & - & • & $\theta^{\circ}$ & 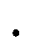 & ${ }^{\circ}$ & 4 \\
\hline AMSA Radioisotope & Caps & sule & - & - & - & • & • & & & 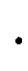 & 5 \\
\hline CAPSULE DEVELOPMENT & PHA & SE I & - & - & - & - & • & • & & $\bullet$ & \\
\hline Design. & - & . & - & - & - & - & • & - & $\cdot$ & • & \\
\hline Powder Preparation & & . & - & - & - & - & • & ${ }^{\circ}$ & 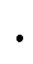 & $\bullet^{\circ}$ & 8 \\
\hline Pressing and Sinte & ring & & . & . & . & - & ${ }^{\circ}$ & $\bullet$ & & 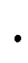 & 8 \\
\hline Cladding Component & & - & - & • & - & - & - & ${ }^{\circ}$ & & • & 12 \\
\hline We1ding Developmen & & - & - & - & - & - & - & $\bullet$ & 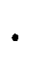 & 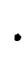 & 17 \\
\hline Primary We1ds & - & - & - & - & - & - & • & ${ }^{\circ}$ & 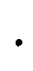 & • & 18 \\
\hline Secondary Welds & $\cdot$ & - & $\cdot$ & - & - & - & ${ }^{\circ}$ & ${ }^{\circ}$ & 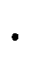 & ${ }^{\circ}$ & 19 \\
\hline Alternate Process & Deve & $1 \mathrm{opm}$ & lent & - & - & - & • & ${ }^{\circ}$ & 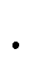 & $\cdot$ & 23 \\
\hline QUAL IFICATION CAPSUL & ES P & PHASE & I I & $\cdot$ & $\cdot$ & $\cdot$ & • & 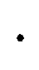 & $\bullet$ & ${ }^{\circ}$ & 25 \\
\hline Impact Tests and $\mathrm{P}$ & osti & $\mathrm{impac}$ & tion & E & alua & tion & • & $\cdot$ & $\bullet$ & $\cdot$ & 25 \\
\hline AMSA HEAT SOURCE FAB & BRICA & ATION & PHA & SE & I I I & - & $\bullet$ & 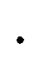 & $\bullet$ & $\bullet$ & 29 \\
\hline Promethium Purific & catio & & - & - & - & - & - & $\bullet$ & $\bullet^{\circ}$ & • & 29 \\
\hline Powder Preparation & & - & - & - & - & - & $\bullet$ & $\bullet$ & $\bullet$ & - & 31 \\
\hline Pressing and Sinte & ring & & - & $\cdot$ & - & - & - & • & $\cdot$ & $\bullet$ & 32 \\
\hline Assemb1y of AMSA $\mathrm{H}$ & Heat & Sour & ce $\mathrm{C}$ & Cap & ule & $\cdot$ & • & $\bullet$ & • & - & 43 \\
\hline HANDLING AND SHIPPIN & & - & - & - & - & - & $\cdot$ & $\cdot$ & • & • & 49 \\
\hline REFERENCES • • & - & - & - & - & - & - & $\bullet$ & $\bullet$ & $\cdot$ & $\bullet$ & 55 \\
\hline ACKNOWLEDGEMENTS. & - & - & - & - & - & - & • & - & - & $\cdot$ & 56 \\
\hline APPENDIX & - & - & • & - & - & - & • & - & • & • & \\
\hline
\end{tabular}




\section{LIST OF FIGURES}

AMSA Thermal Power Capsule

$\mathrm{Sm}_{2} \mathrm{O}_{3}$ Pellets

Split Sleeve Die

AMSA Pellet and Cladding Assembly Components 12

Electron Beam Welder

Typical Tantalum Electron Beam Seal Weld

L-605 Phase II Secondary Cladding Weld, Showing Optimized Penetration

HERF Die and Punches

Double Clad Capsule High-Energy Rate Formed, Ready for Final Machining

$45^{\circ}$ Impact Failure

$45^{\circ}$ Impact Failure as Sectioned

AMSA Capsule After Impact Against Concrete at $678 \mathrm{ft} / \mathrm{sec}$

AMSA Radioisotope Encapsulation Process 30

${ }^{147} \mathrm{Pm}_{2} \mathrm{O}_{3}$ Powder Container

${ }^{147} \mathrm{Pm}_{2} \mathrm{O}_{3}$ Modified Die

${ }^{147} \mathrm{Pm}_{2} \mathrm{O}_{3}$ Glove Box with Press Attached 36

${ }^{147} \mathrm{Pm}_{2} \mathrm{O}_{3}$ Sintering Furnace

36

Promethium Pellet No. 1 as Pressed

37

Promethium Pellet No. 2 as Sintered

Promethium Pellet No. 1 Recovery Attempt

Promethium Pellet No. 2 as Sintered 41

Promethium Pellet No. 3 as Assembled 43

AMSA Capsule Inner C1ad Welded 44

Inner Tantalum Weld, Test Capsule 45

Nondestructive Test Profile of Fueled Capsule 46

Outer L-605 Weld Test Capsule 47

Two Views of Final AMSA Fueled Capsule 50

Radiation Mapping $\quad 51$

2R ICC Specified Container with Holding Fixture 52

Shipping Barre1 
31 Radiation and Temperature Profile of Shipping Container

32 Radiation and Temperature Profile of $2 \mathrm{R}$ Container

A-1 Gamma Spectrum of $60 \mathrm{~W},{ }^{147} \mathrm{Pm}_{2} \mathrm{O}_{3}$ AMSA Capsule A-7 


\section{LIST OF TABLES}

1

$\mathrm{Sm}_{2} \mathrm{O}_{3}$ Pellets Pressing and Sintering Data

Final Assembly Data on $\mathrm{Sm}_{2} \mathrm{O}_{3}$ Capsules

Types of Quality Assurance Inspections

Fueled Capsule Component Test Results

A -1

Fueled Capsule Pellet Test Results 
.

. 
BNWL -994

\section{PROMETHIUM-147 RADIOISOTOPE APPLICATION PROGRAM \\ AMSA HEAT SOURCE \\ FINAL REPORT}

N. C. Davis and D. W. Brite

\section{INTRODUCTION}

Batte11e-Northwest (Batte1le Memorial Institute/Pacific Northwest Laboratory) was requested to design and fabricate a radioisotope heat source for an Air Force application involving the Advanced Manned Strategic Aircraft (AMSA). In this application, a promethium-147 heat source was proposed for use in a thermal preconditioner to maintain the navigation system at an elevated temperature.

The program required close coordination among personnel from the Air Force, AC Electronics (overall systems responsibilities), AEC Division of Isotope Development, BattelleNorthwest (capsule responsibility), and Sandia Laboratory (safety analyses and testing).

This document is the final report on the AMSA capsule development and fabrication program conducted by Batte1leNorthwest.

The AMSA Radioisotope Capsule program was divided into three phases. The objectives of the program are listed under each phase as follows:

Phase I Capsule Development

- Prepare six to $10 \mathrm{Sm}_{2} \mathrm{O}_{3}$ fueled capsules using two candidate fabrication processes: pressing and sintering, and high energy-rate forming.

- Impact test the $\mathrm{Sm}_{2} \mathrm{O}_{3}$ fueled capsules at Sandia to determine design and process suitability. 
Phase II Capsule Qualification

- Refine capsule design, process parameters, and evaluation techniques based on Phase I results.

- Prepare 20 to $30 \mathrm{Sm}_{2} \mathrm{O}_{3}$ fueled capsules.

- Impact test $20 \mathrm{Sm}_{2} \mathrm{O}_{3}$ fueled capsules at Sandia for final qualification.

Phase II I AMSA ${ }^{147} \mathrm{Pm}_{2}{ }_{3}$ Capsule

- Fabricate and deliver to the Air Force - one, 65 thermal watt ${ }^{147} \mathrm{Pm}_{2} \mathrm{O}_{3}$ heat source for use in the AMSA application.

The major divisions in this final report correspond to the major divisions in the program (i.e., Phases I, II, and II). A final section has been added to illustrate handling and shipping procedures for the final heat source. Process development of the test capsules is discussed, destructive evaluation of the impact capsules is summarized, and special emphasis is placed on the Phase III final fabrication and quality assurance of the promethium-fueled heat source capsule. 


\section{SUMMARY}

\section{CAPSULE DEVELOPMENT}

Promethium-147 was selected as the most desirable isotope due to low radiation dose rate with minimal shielding, fuel stability, and adequate power density in the designed size. The allowable design flexibility permitted a heavy wall, double clad pellet assembly that could be fabricated either by slip-fit assembly of sintered pellets or by High Energy Rate Forming (HERF) of the assembly.

The final capsule design and development resulted in a nearly-spherical (dome ends for impact resistance), 2 in. diameter and 2 in. long heat source with double containment cladding. The inner (primary) clad was fabricated from tantalum to provide radiation shielding, and the outer (secondary) clad was machined from L-605 (cobalt alloy) bar stock for high strength impact resistance. Preliminary process development and impact test results indicated that either the slip-fit assembly process or the high energy rate forming (HERF) process would be suitable in this fabrication program. The slip-fit assembly method was selected since the process was easier to develop and the simple approach was considered adequate for this application. The usual advantages of the HERF method, e.g., intimate contact between core and all claddings and higher core densities, were not considered necessary for this application.

Both the production and evaluation of the many process and impact test samples required in the development and qualification of an isotope heat source are simplified by using a nonradioactive stand-in applicable to the final heat source fuel form. Samarium oxide was chosen as a stand-in for the highly radioactive promethium because its chemical and physical 
properties are very similar. Another pertinent consideration in the selection of a stand-in is the fact that samarium-147 is the stable product formed when promethium-147 decays.

\section{CAPSULE QUALIFICATION}

In the capsule development phase, the fabrication and evaluation of 10 capsules resulted in the capsule design and fabrication process selection. An additional 30 capsules were fabricated during the qualification phase of the program. Twenty of these were impact tested at the Sandia Laboratories. The total test program has shown:

- The capsule may reasonably be expected to provide fue 1 containment for all except the most adverse impact conditions. (1)

- Capsule terminal velocity as determined by calculations and experimental drop tests is approximately $340 \mathrm{ft} / \mathrm{sec}$.

- A critical impact angle exists at an altitude of $45^{\circ}$ from the cylindrical axis of the capsule. The critical angle was determined experimentally by rocket sled impact tests against a hard granite target.

- At or near the critical impact angle, capsules impacted against granite at velocities as great as $275 \mathrm{ft} / \mathrm{sec}$. remained intact.

- At other impact angles $\left(0^{\circ}, 67-1 / 2^{\circ}\right.$, and $\left.90^{\circ}\right)$ against the hard granite target, the capsule did not fail at impact velocities up to about $500 \mathrm{ft} / \mathrm{sec}$.

- At the critical impact angle, using 6000 psi compressive strength concrete as the target, capsules tested at $400 \mathrm{ft} / \mathrm{sec}$ and at $675 \mathrm{ft} / \mathrm{sec}$ were only superficially scratched, although the concrete targets were destroyed.

- At a random impact angle against a soil target, a single test at $625 \mathrm{ft} / \mathrm{sec}$ also resulted in on $1 \mathrm{y}$ superficial scratching of the capsule. 
BNWL -994

- Post-testing evaluations indicated a high degree of capsule integrity, and thus a sound design and reproducible fabrication processes. The only impact failures were attributed to the 40,000 psi compressive strength granite targets at or near the critical impact angle. All capsules survived impacts against concrete and soil targets .

\section{AMSA RADIOISOTOPE CAPSULE}

A detailed process and quality assurance plan based on process technology established during the qualification phase of this program was placed in effect for the fabrication of the promethium fueled AMSA heat source. In working with the radioactive isotope, contamination and radiation problems were minor, but fabrication difficulties were encountered due to the self-heating characteristics of promethium. Progressive improvements were made in three attempts to produce a suitable one-piece pellet for the AMSA capsule. However, in each case, thermal gradients within the pressed pellet caused it to fracture and the final assembly was made with a three-piece pellet. Although assembly of the capsule was made more difficult by having to stack the three-piece pellet, the physical integrity of the completed capsule was not compromised.

The AMSA process flow diagram and quality control procedures were followed very closely in making the double cladding assembly. After decontamination of the primary cladding was effected and the first weld was made, the capsule was nonsmearable for the balance of the assembly process. A detailed quality control inspection of the fueled capsule indicated that its integrity was equivalent to that of the qualification impact test capsules. The high degree of reliability developed for the nondestructive testing procedures used in making this determination was achieved by careful destructive evaluation of all of the impacted test capsules and correlation of the observed cladding integrity with the nondestructive testing data. 
BNWL -994

\section{CAPSULE DEVELOPMENT PHASE I}

A promethium-147 heat source capsule was fabricated for the Air Force to be used in a Thermal Preconditioning Unit (TPU) within the Advanced Manned Strategic Aircraft (AMSA). The TPU, a piece of auxiliary support equipment for an Inertial Measuring Unit (IMU) was designed to provide an improved navigation system reaction capability. The TPU reduces the time interval between the application of power to the navigation system and the attainment of specified performance by maintaining the navigation system at an elevated temperature. To accomplish this function, a promethium-147 source of 65 thermal watts initial power was designed, developed, and flight qualified.

\section{DESIGN}

The basic capsule dimensions were fixed by the TPU design, while the shape, including the dome ends, was designed to optimize impact resistance (Figure 1). Promethium-147 met the basic requirements of geometry, weight, and safety for the application and was considered a desirable isotope due to:

- A very low dose rate with only minimal shielding (beta emitter).

- Appropriate half-life.

- Adequate power density of the stable sesquioxide fuel form.

- Minimal design problems due largely to a demonstrated capability of producing heat sources of this size.

- Projected availability for potential scaleup requirements.

Tantalum, because of its shielding characteristics and its relative ease of fabrication was selected as the inner cladding. Tantalum radiation shielding 0.060 in. in thickness reduced the dose rate to 10 to $30 \mathrm{mR} / \mathrm{hr}$ at $1 \mathrm{~m}$. A uniform $0.200 \mathrm{in}$. thick wall of L-605 alloy for the secondary outer cladding was 


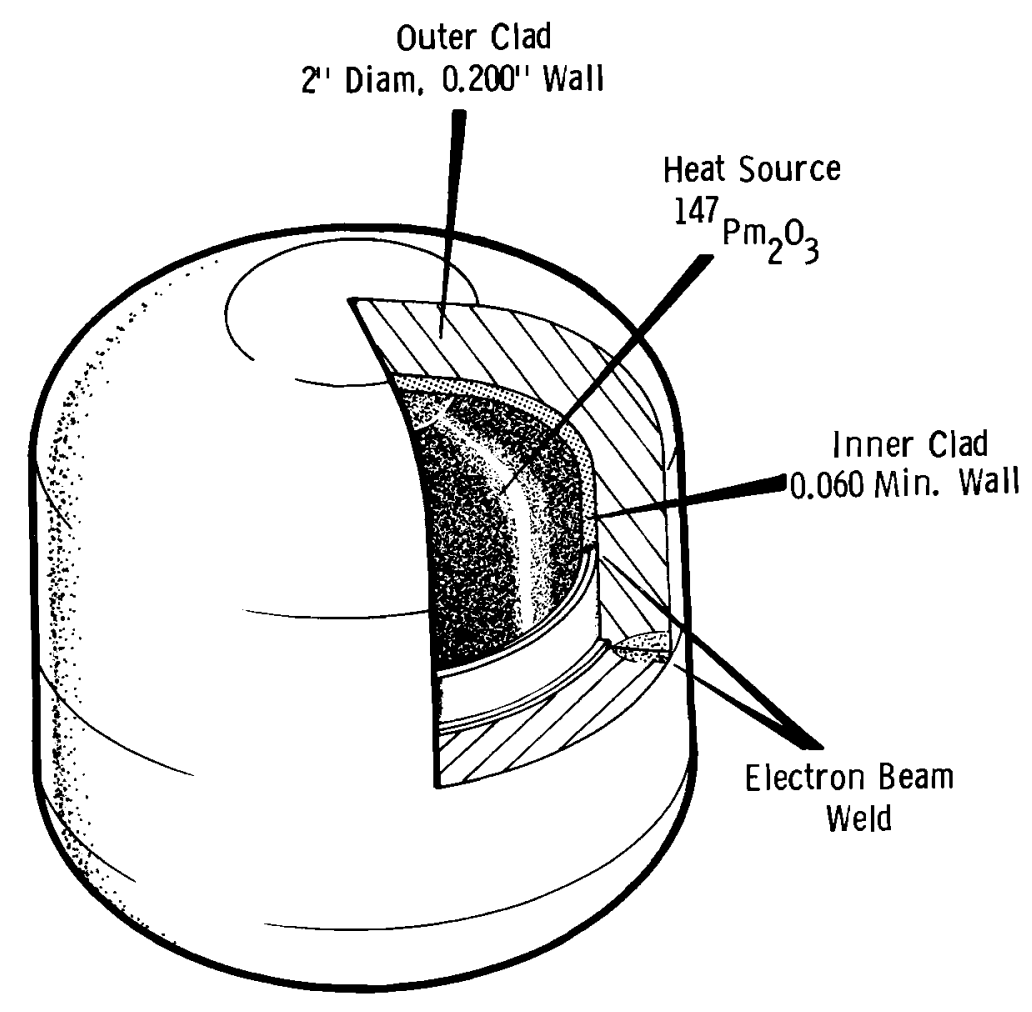

Neg 0681559

FIGURE 1. AMSA Thermal Power capsule

selected to maximize impact strength within limits dictated by the total capsule size, by the fuel core volume, and by shielding requirements. This high strength cobalt alloy has outstanding corrosion resistance.

Another pertinent consideration in the capsule design was the weld location. The primary and secondary welds were offset from each other to minimize any possible shear plane of weakness through the capsule (Figure 1). The primary weld, intended only as a contamination seal, was offset from the center of the capsule. The secondary weld, which required the optimum impact resistance, was located at the center of the longitudinal axis of the capsule. This center location, 
BNWL - 994

because of the capsule's dome end shape, afforded the greatest protection regardless of impact attitude.

This particular design resulted in an overall capsule size 2 in. in diameter and 2 in. long, with dome ends on a 0.645 in. outside radius. Dimensions of the inner capsule were chosen to allow adequate radiation protection and sufficient clearance for easy assembly while providing the maximum practical fuel volume. The resulting volume for the isotope, approximately $37 \mathrm{~cm}^{3}$, was adequate to provide the specified $65 \mathrm{~W}$ of thermal power using freshly purified promethium-147 oxide at a density with relative ease to 90 to $95 \%$ of theoretical density.

\section{POWDER PREPARATION}

Samarium oxide was used in all the development and qualification prototype capsules. A comparison between the physical and chemical characteristics of samarium oxide and promethium oxide indicated that processing results of both materials would be comparable except for the decay heating of the latter. Purity of the samarium, as received, was $99.0 \%$. Prior to its use in the capsule development program, the oxide was subjected to further purification treatment similar to that used in preparing high purity promethium-147.

Additional powder processing included a consolidation step consisting of pressing the powder into pellets and then crushing them into agglomerated powder. The powder was then calcined for at least $4 \mathrm{hr}$ at $1100{ }^{\circ} \mathrm{C}$ to stabilize the monoclinic form. After this treatment, the powder showed a tap density of approximately $30 \%$ of theoretical. Theoretical density of monoclinic $\mathrm{Sm}_{2} \mathrm{O}_{3}$ is $7.74 \mathrm{~g} / \mathrm{cm}^{3}$.

PRESSING AND SINTERING

The original fuel core concept was based on a stack of ${ }^{147} \mathrm{Pm}_{2} \mathrm{O}_{3}$ pellets. These pellets could be pressed and sintered with relative ease to 90 to $95 \%$ of theoretical density. 
The first series of $10 \mathrm{Sm}_{2} \mathrm{O}_{3}$ capsules in Phase I was produced by the use of stacked pellets. Since handling problems were anticipated with the radioactive pellets, effort was made to increase the size of the individual pellets and thus minimize the number required for the $65 \mathrm{~W}$ thermal power source. $\mathrm{Sm}_{2} \mathrm{O}_{3}$ capsule loads consisting of two pellets were produced in Phase I and, in Phase II, one-piece double dome pellets were produced (Figure 2).

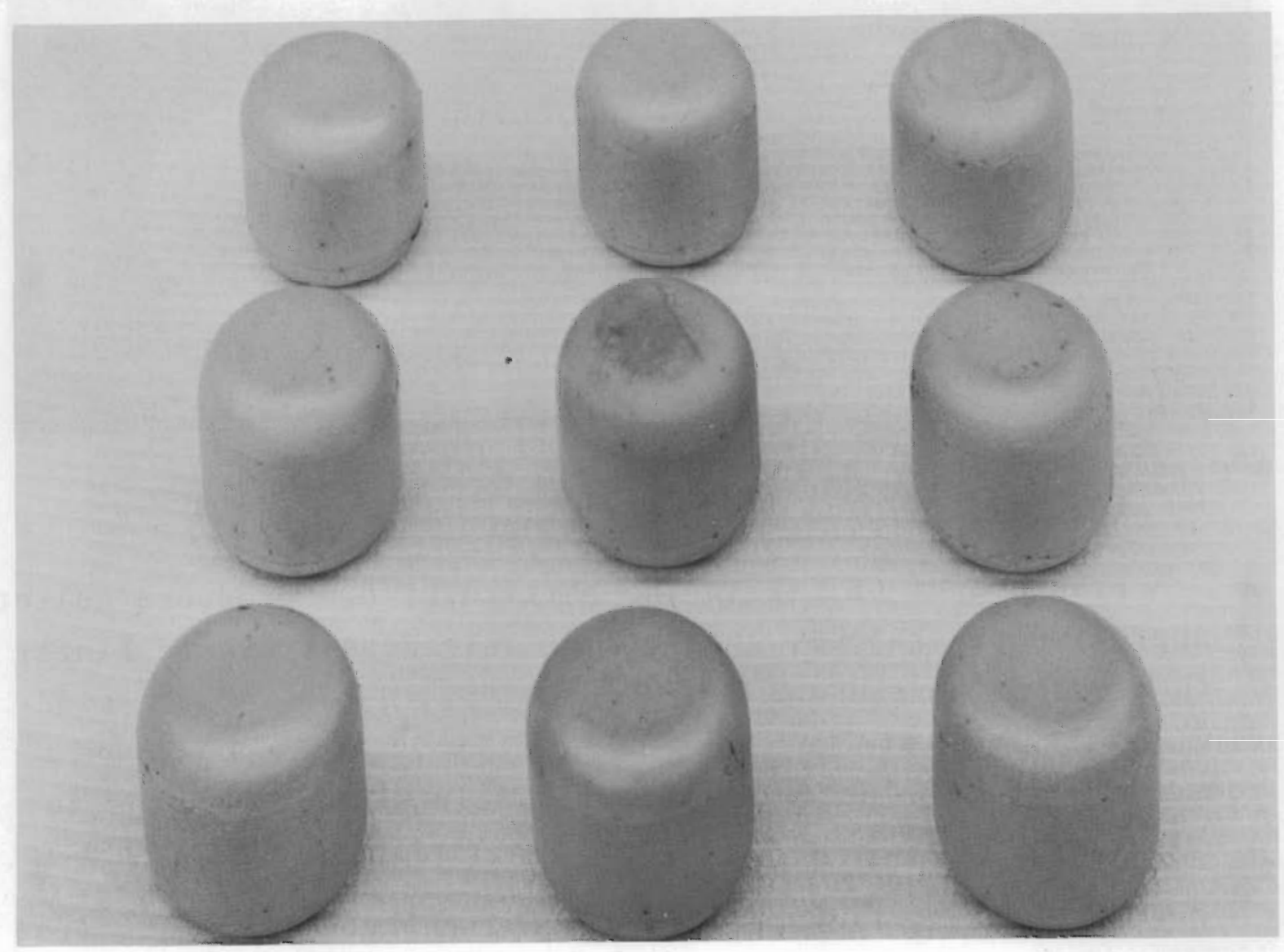

Neg $0672194-3$

FIGURE 2. $\mathrm{Sm}_{2} \mathrm{O}_{3}$ Pellets (stand-in fuel)

The punch and die set (Figure 3) includes a tapered split sleeve which makes it possible to press and eject pellets without the addition of an organic binder. 


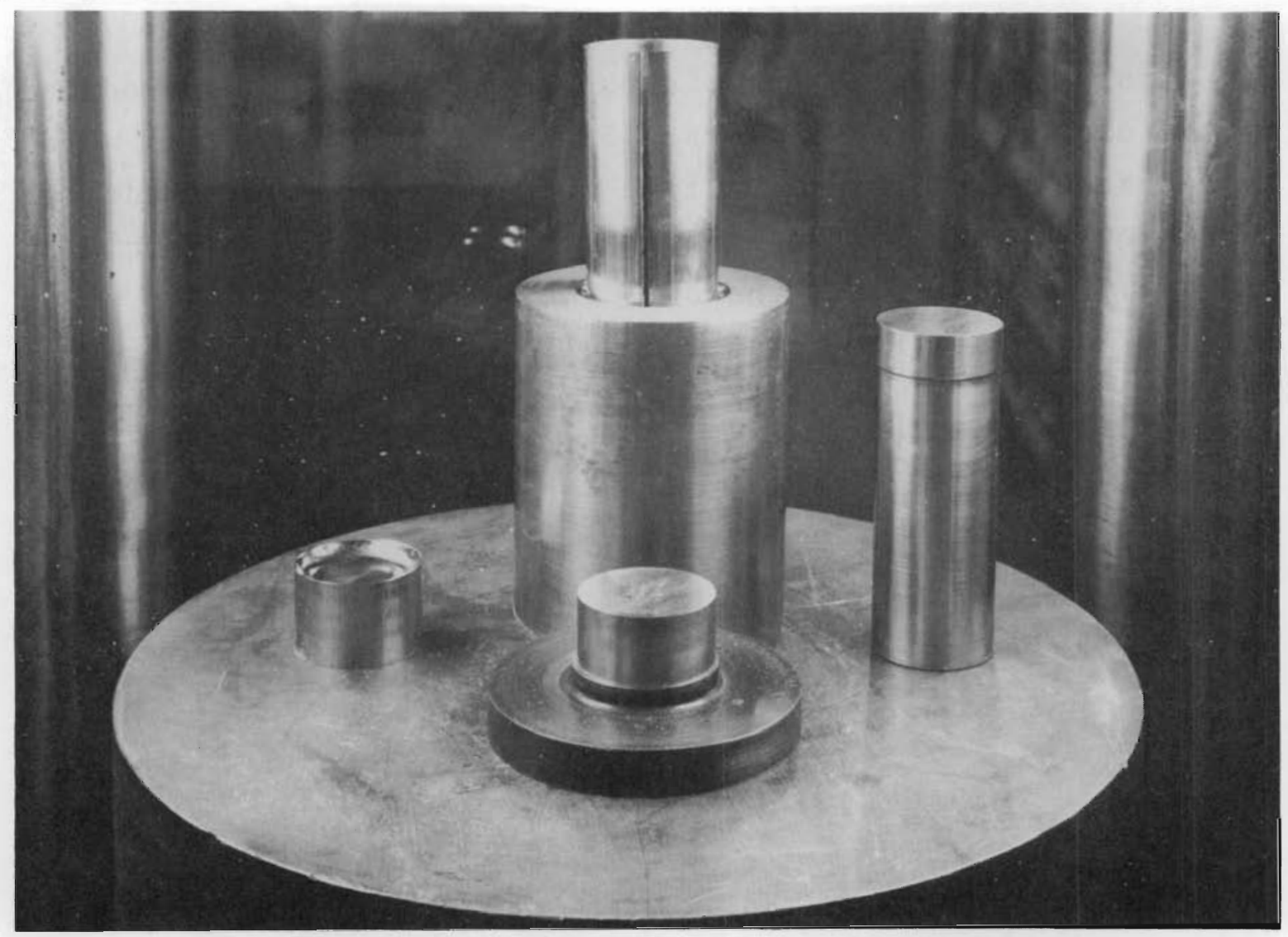

Neg 0670660-8

\section{FIGURE 3. Split Sleeve Die}

A pressure of $65 \mathrm{tsi}$ in the particular die produced pellets of approximately $75 \% \mathrm{TD}$. Pellets of this density would sinter to the correct length and diameter to fit in the capsule cladding. It was determined that $12 \mathrm{hr}$ at 1350 to $1400{ }^{\circ} \mathrm{C}$ would reduce the pressed pellets at least $7 \%$ in diameter and $6 \%$ in length, and result in a final density of approximately 95\% TD.

Table 1 summarizes the dimensional changes resulting from sintering the $\mathrm{Sm}_{2} \mathrm{O}_{3}$ pellets. In this as well as in subsequent tables, the identification system was continued (with No. 11 forward) to include all the pellets fabricated for the qualification phase of the program. The capsule assembly number noted in Table 1 applies to the final capsule identification number 
BNWL - 994

TABLE 1. $\mathrm{Sm}_{2} \mathrm{O}_{3}$ Pellets Pressing and Sintering Data

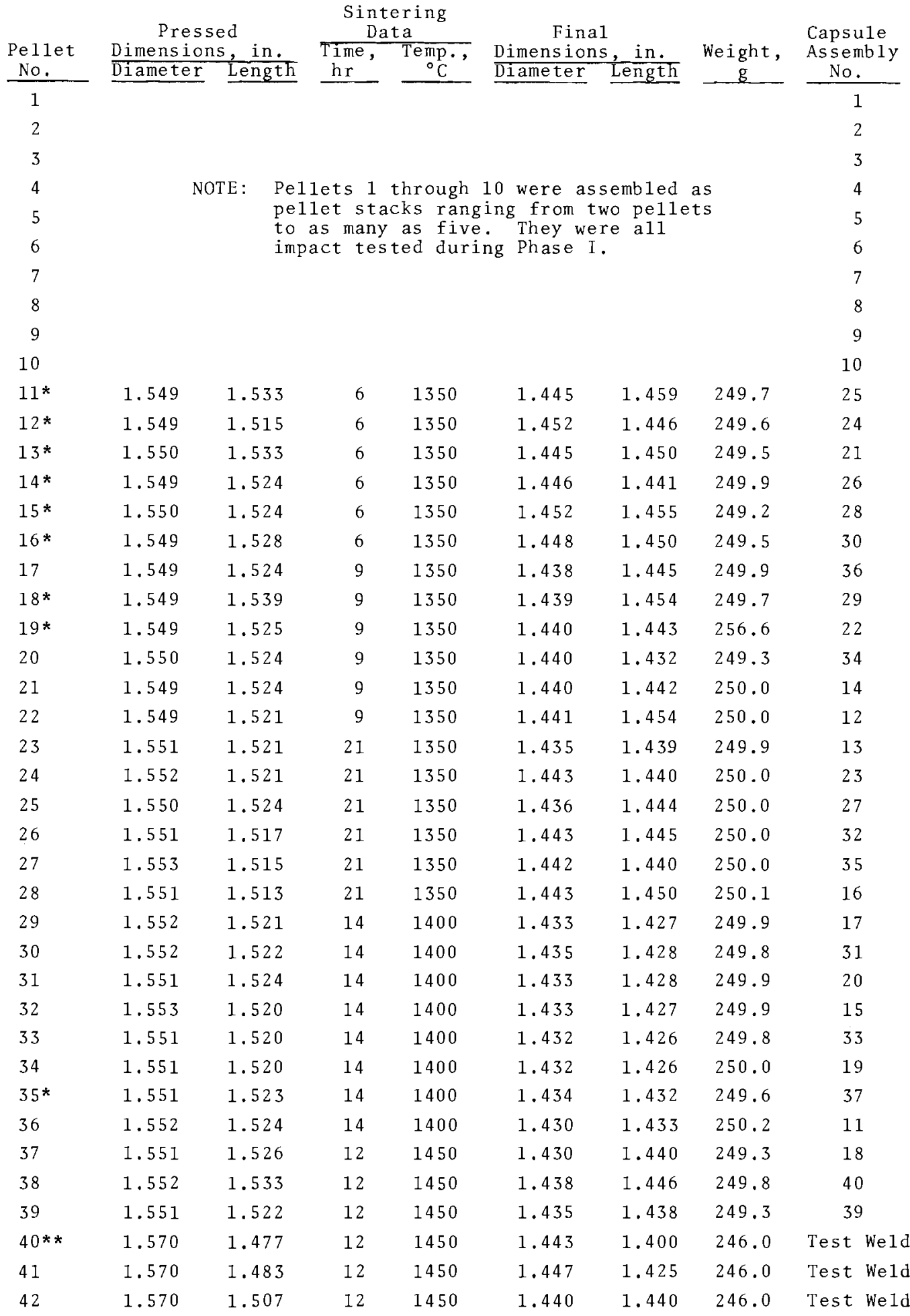

\footnotetext{
* Pellets with broken domes. Other pellets were one-piece. However, micro cracks were evident in many of the single-piece pellets.

** Phase III - Larger diameter die
} 
used throughout the program. All additional tables in this report containing component data and final impact test results carry the same capsule assembly number. These tables thus provide a complete fabrication record of the components used in each capsule, as well as a final postimpaction evaluation.

\section{CLADDING COMPONENTS}

Both the tantalum primary cladding and the L-605 alloy secondary cladding were machined from bar stock. Cladding and pellet assembly components are shown in Figure 4. Dimensional inspection data of primary and secondary claddings are given in Tables 2 and 3 , respectively. Final assembly data on all $\mathrm{Sm}_{2} \mathrm{O}_{3}$ developmental capsules are given in Table 4.

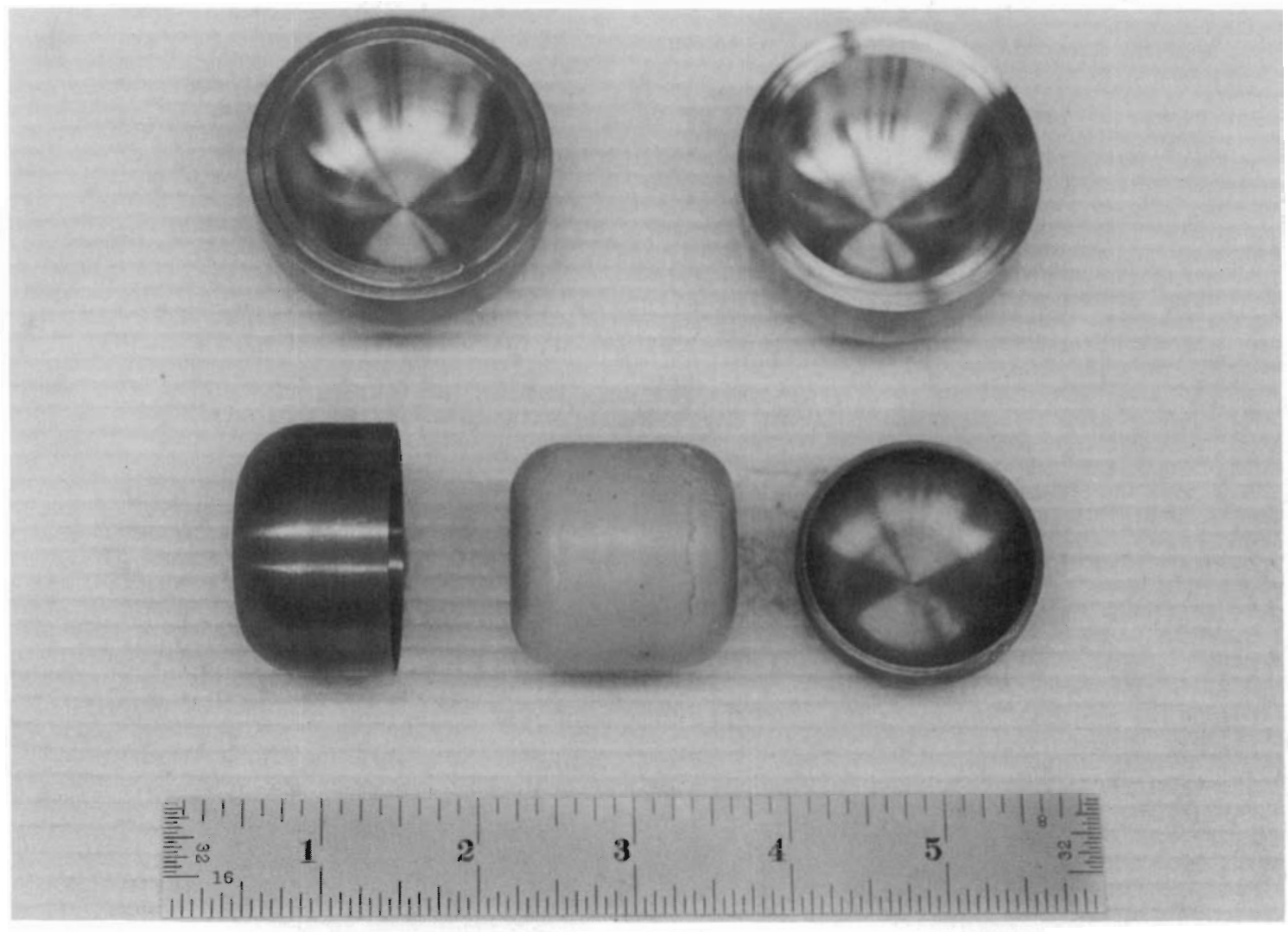

Neg $0671707-2$

FIGURE 4. AMSA Pellet and Cradding Assembly Components 


\begin{tabular}{|c|c|c|c|c|c|c|c|c|}
\hline & $\varepsilon^{\bullet} 99 \mathrm{I}$ & $890^{\circ} 0$ & $\tau \angle 0^{\circ} 0$ & $09 t^{\circ} \mathrm{I}$ & {$\left[9 t^{\circ} \mathrm{L}\right.$} & $\angle 6 \mathrm{~S}^{\circ} \mathrm{I}$ & $66 S^{\circ} \mathrm{I}$ & $49 t$ \\
\hline & $t \cdot t 9 I$ & $\angle 90^{\circ} 0$ & $0 \angle 0^{\circ} 0$ & $8 \mathrm{St} \cdot \mathrm{I}$ & $09 t^{\circ} \mathrm{I}$ & $S 6 S^{\circ} \mathrm{I}$ & $86 \mathrm{~S}^{\circ} \mathrm{I}$ & $s t$ \\
\hline & $6^{\circ} 8 \mathrm{SI}$ & $\angle 90^{\circ} 0$ & $690^{\circ} 0$ & $6 S t^{\circ} I$ & $\varepsilon 9 t^{\circ} I$ & $009^{\circ} \mathrm{I}$ & $009^{\circ} \mathrm{I}$ & $\nabla t$ \\
\hline $7 \mathrm{~S} \partial \mathrm{L}$ & $8 \cdot 09 \mathrm{I}$ & $990^{\circ} 0$ & $0 \angle 0^{\circ} 0$ & $8 S t^{\circ} I$ & $29 t^{\circ} I$ & $86 S^{\circ} I$ & $009^{\circ} \mathrm{I}$ & $\varepsilon \downarrow$ \\
\hline & $L \cdot 09 \mathrm{I}$ & $990^{\circ} 0$ & $690^{\circ} 0$ & $6 S t^{\circ} I$ & $79 t^{\circ} \tau$ & $66 \mathrm{~S}^{\circ} \mathrm{I}$ & $66 \mathrm{~S}^{\circ} \mathrm{I}$ & $2 t$ \\
\hline & $9^{\bullet} \varsigma 9 \mathrm{I}$ & $890^{\circ} 0$ & $2 \angle 0^{\circ} 0$ & $\angle S t^{\circ} I$ & $8 S t^{\circ} \mathrm{I}$ & $\angle 6 S^{\circ} I$ & $96 S^{\circ} \mathrm{T}$ & $I t$ \\
\hline $0 \mathrm{t}$ & $0.0 \angle I$ & $890^{\circ} 0$ & $0 \angle 0^{\circ} 0$ & $6 s t^{\circ} I$ & $09 t^{\circ} \mathrm{I}$ & $865^{\circ} \mathrm{I}$ & $86 \mathrm{~g}^{\circ} \mathrm{T}$ & $0 t$ \\
\hline $6 \varepsilon$ & $0 \cdot 0 \angle \mathrm{I}$ & $\angle 90^{\circ} 0$ & $2 \angle 0^{\circ} 0$ & $9 S t^{\circ} I$ & $\mathrm{~L} 9 \mathrm{t}^{\circ} \mathrm{I}$ & $66 \mathrm{~S}^{\circ} \mathrm{I}$ & $S 6 S^{\circ} I$ & $6 \varepsilon$ \\
\hline PIәM $75 \partial L$ & $S \cdot 89 I$ & $\angle 90^{\circ} 0$ & $\tau \angle 0^{\circ} 0$ & $6 S t^{\circ} I$ & $09 t^{\circ} \mathrm{I}$ & $009^{\circ} \mathrm{I}$ & $96 \mathrm{C}^{\cdot} \mathrm{I}$ & $8 \varepsilon$ \\
\hline $9 \varepsilon$ & $S \cdot 89 \mathrm{I}$ & $890^{\circ} 0$ & $2 \angle 0^{\circ} 0$ & $8 \mathrm{St} \cdot \mathrm{L}$ & $6 S t^{\circ} \mathrm{I}$ & $009^{\circ} \mathrm{I}$ & $96 S^{*} \mathrm{I}$ & $\angle \varepsilon$ \\
\hline 82 & $S \cdot Z \angle I$ & $\angle 90^{\circ} 0$ & $0 \angle 0^{\circ} 0$ & $09 t^{\circ} I$ & $09 t^{\circ} I$ & $66 S^{\circ} I$ & $96 S^{\circ} \mathrm{T}$ & $9 \varepsilon$ \\
\hline $2 \varepsilon$ & $0^{\bullet} \mathrm{T} \angle \mathrm{T}$ & $\angle 90^{\circ} 0$ & $2 \angle 0^{\circ} 0$ & $6 S t^{\circ} \mathrm{I}$ & $09 t^{\circ} \mathrm{I}$ & $009^{\circ} \mathrm{L}$ & $\angle 6 \mathrm{~S}^{\circ} \mathrm{I}$ & $s \varepsilon$ \\
\hline$\nabla \varepsilon$ & $S \cdot 99 I$ & $890^{\circ} 0$ & $\mathrm{~L} \angle 0^{\circ} 0$ & $09 t^{\circ} \mathrm{I}$ & $6 \mathrm{st} \mathrm{t}^{\circ} \mathrm{I}$ & $009^{\circ} \mathrm{L}$ & $\angle 6 S^{\circ} \mathrm{I}$ & $t \varepsilon$ \\
\hline$\varepsilon \varepsilon$ & $S \cdot 89 I$ & $\angle 90^{\circ} 0$ & $\tau \angle 0^{\circ} 0$ & $8 \mathrm{St} \mathrm{t}^{\circ} \mathrm{L}$ & $6 \mathrm{St} \cdot \mathrm{I}$ & $009^{\circ} \mathrm{I}$ & $96 \mathrm{~S}^{\circ} \mathrm{I}$ & $\varepsilon \varepsilon$ \\
\hline$\angle \varepsilon$ & $S^{\cdot} 0 \angle L$ & $\angle 90^{\circ} 0$ & $\mathrm{I} \angle 0^{\circ} 0$ & $09 t^{\circ} \mathrm{L}$ & $6 S t^{\circ} \mathrm{I}$ & I09 I & $96 \mathrm{~S}^{*} \mathrm{~T}$ & $2 \varepsilon$ \\
\hline IS & $0^{\circ} \mathrm{L} \angle \mathrm{I}$ & $690^{\circ} 0$ & $2 \angle 0^{\circ} 0$ & $8 \mathrm{St}^{\circ} \mathrm{L}$ & $09 \mathrm{~V}^{\circ} \mathrm{I}$ & I09. I & $\angle 6 S^{\circ} \mathrm{I}$ & {$[\varepsilon$} \\
\hline $0 \varepsilon$ & $S^{\circ} T \angle T$ & $890^{\circ} 0$ & $\mathrm{~T} \angle 0^{\circ} 0$ & $09 t^{\circ} \mathrm{I}$ & $6 \mathrm{St} \mathrm{t}^{\circ} \mathrm{I}$ & I09 $\mathrm{I}$ & $865^{\circ} \mathrm{T}$ & $0 \varepsilon$ \\
\hline Iz & $S^{\circ} 0 \angle I$ & $\angle 90^{\circ} 0$ & $\varepsilon \angle 0^{\circ} 0$ & $\operatorname{sst} t^{\circ} I$ & $09 \mathrm{~V}^{\circ} \mathrm{I}$ & $009^{\circ} \mathrm{I}$ & $96 S^{\circ} \mathrm{I}$ & 62 \\
\hline $6 \mathrm{I}$ & $0^{\circ} \mathrm{T} \angle \mathrm{I}$ & $890^{\circ} 0$ & $2 \angle 0^{\circ} 0$ & $\angle S t^{\circ} I$ & $6 S t^{\circ} \mathrm{I}$ & I $09^{\circ} \mathrm{I}$ & $86 \mathrm{~S}^{\circ} \mathrm{I}$ & 82 \\
\hline 62 & $0.0 \angle \mathrm{T}$ & $\angle 90^{\circ} 0$ & $\mathrm{I} \angle 0^{\circ} 0$ & $09 t^{\circ} \mathrm{I}$ & $6 \mathrm{St} \mathrm{t}^{\circ} \mathrm{I}$ & $209^{\circ} \mathrm{I}$ & $\angle 6 S^{\circ} \mathrm{T}$ & $\angle Z$ \\
\hline $9 z$ & $0^{\circ} \mathrm{T} \angle \mathrm{T}$ & $890^{\circ} 0$ & $\varepsilon \angle 0^{\circ} 0$ & $8 S t^{\circ} \mathrm{I}$ & $09 t^{\circ} \mathrm{I}$ & $\varepsilon 09^{\circ} \mathrm{I}$ & $\angle 6 S^{\circ} \mathrm{T}$ & $9 z$ \\
\hline$\subseteq \varsigma$ & $S^{\circ} \angle 9 I$ & $\angle 90^{\circ} 0$ & $\varepsilon \angle 0^{\circ} 0$ & $6 S t^{*} \mathrm{I}$ & $\tau 9 t^{\circ} \tau$ & $209^{\circ} \mathrm{I}$ & $\angle 6 S^{\circ} \mathrm{I}$ & $s z$ \\
\hline 12 & $0^{\circ} Z \angle I$ & $890^{\circ} 0$ & $\varepsilon \angle 0^{\circ} 0$ & $6 S t^{\circ} \mathrm{I}$ & $6 \mathrm{St} \cdot \mathrm{T}$ & $\varepsilon 09^{\circ} \mathrm{I}$ & $86 \mathrm{~S}^{\circ} \mathrm{T}$ & $t 2$ \\
\hline$s z$ & $s^{\circ} \angle 9 I$ & $\varsigma 90^{\circ} 0$ & $2 \angle 0^{\circ} 0$ & $\varepsilon 9 t^{\circ} \mathrm{I}$ & $09 \mathrm{t}^{\circ} \mathrm{I}$ & $009^{\circ} \mathrm{I}$ & $96 \mathrm{~S}^{\circ} \mathrm{T}$ & $\varepsilon \tau$ \\
\hline $8 \mathrm{I}$ & $\mathrm{S}^{\cdot} 0 \angle \mathrm{I}$ & $890^{\circ} 0$ & $\varepsilon \angle 0^{\circ} 0$ & $\angle S V^{\circ} I$ & $09 \mathrm{t}^{\circ} \mathrm{I}$ & I09. I & $86 \mathrm{~S}^{\circ} \mathrm{I}$ & 22 \\
\hline $2 z$ & $s^{\circ} 0 \angle I$ & $690^{\circ} 0$ & $\varepsilon \angle 0^{\circ} 0$ & $\operatorname{sst} 0^{\circ} \mathrm{I}$ & $09 t^{\circ} \mathrm{I}$ & $009^{\circ} \mathrm{I}$ & $86 \mathrm{~S}^{\circ} \mathrm{I}$ & {$[z$} \\
\hline $0 z$ & $0^{\circ} 69 \mathrm{I}$ & $690^{\circ} 0$ & $\forall \angle 0^{\circ} 0$ & $8 \mathrm{St} \mathrm{t}^{\circ} \mathrm{I}$ & $8 \mathrm{St} \mathrm{t}^{\circ} \mathrm{I}$ & I09. I & $86 \mathrm{~S}^{\circ} \mathrm{T}$ & 02 \\
\hline$S \mathrm{I}$ & $\varepsilon \cdot 29 I$ & $990^{\circ} 0$ & $\nabla \angle 0^{\circ} 0$ & $8 \mathrm{St} \mathrm{t}^{\circ} \mathrm{I}$ & $09 t^{\circ} \mathrm{L}$ & $009^{\circ} \mathrm{I}$ & $06 \mathrm{~S}^{\circ} \mathrm{T}$ & $6 \mathrm{I}$ \\
\hline$\angle Z$ & $9^{\circ} \mathrm{S} 9 \mathrm{I}$ & $990^{\circ} 0$ & $t \angle 0^{\circ} 0$ & $\operatorname{sst} t^{\circ} \mathrm{I}$ & $6 \mathrm{St} \mathrm{t}^{\circ} \mathrm{I}$ & $009^{\circ} \mathrm{I}$ & $S 6 S^{\circ} \mathrm{I}$ & $8 \mathrm{I}$ \\
\hline$\angle I$ & $8^{\circ}+9 I$ & $890^{\circ} 0$ & {$\left[\angle 0^{\circ} 0\right.$} & $6 \mathrm{St} \cdot \mathrm{I}$ & $85 \mathrm{t}^{\circ} \mathrm{I}$ & $669^{\circ} \mathrm{I}$ & $96 \mathrm{~S} \cdot \mathrm{I}$ & $\angle I$ \\
\hline $9 \mathrm{~T}$ & $0^{\circ} \varsigma 9 \mathrm{I}$ & $890^{\circ} 0$ & $\mathrm{~T} \angle 0^{\circ} 0$ & $09 t^{\circ} \mathrm{I}$ & $6 \mathrm{St} \mathrm{t}^{\circ} \mathrm{T}$ & $009^{\circ} \mathrm{I}$ & $\angle 6 S^{\circ} \mathrm{T}$ & $9 \mathrm{I}$ \\
\hline$\varepsilon z$ & $\mathrm{I} \cdot 99 \mathrm{I}$ & $990^{\circ} 0$ & $\varepsilon \angle 0^{\circ} 0$ & $09 t^{\circ} \mathrm{I}$ & $09 t^{\circ} \mathrm{I}$ & $\nabla 09^{\circ} \mathrm{I}$ & $96 \mathrm{~S}^{\circ} \mathrm{T}$ & $S I$ \\
\hline I I & $S^{\prime} S S I$ & $\angle 90^{\circ} 0$ & $0 \angle 0^{\circ} 0$ & $09 \mathrm{~V}^{\circ} \mathrm{I}$ & $6 \mathrm{St} \cdot \mathrm{I}$ & $66 \mathrm{~S}^{\circ} \mathrm{I}$ & $\varepsilon 6 \mathrm{~S}^{\circ} \mathrm{I}$ & $t I$ \\
\hline$\varepsilon I$ & $\tau \cdot \angle 9 I$ & $690^{\circ} 0$ & $\mathrm{I} \angle 0^{\circ} 0$ & $\varepsilon 90^{\circ} \mathrm{I}$ & $6 \mathrm{St}^{\circ} \mathrm{I}$ & $109^{\circ} \mathrm{I}$ & $\angle 6 \mathrm{~S}^{\circ} \mathrm{I}$ & $\varepsilon I$ \\
\hline $2 \mathrm{I}$ & $6^{\circ} 29 \mathrm{I}$ & $890^{\circ} 0$ & $\mathrm{I} \angle 0^{\circ} 0$ & $6 S t^{\circ} \mathrm{I}$ & $\tau 9 t^{\circ} \mathrm{T}$ & $66 \mathrm{~S}^{\circ} \mathrm{I}$ & $96 \mathrm{~S}^{\circ} \mathrm{T}$ & $2 \mathrm{I}$ \\
\hline$t \mathrm{I}$ & $0^{\circ} \varsigma 9 \mathrm{I}$ & $\angle 90^{\circ} 0$ & $\mathrm{I} \angle 0^{\circ} 0$ & $\mathrm{~L} 9 t^{\circ} \mathrm{I}$ & $09 t^{\circ} \mathrm{I}$ & I09. I & $S 6 S^{\circ} \mathrm{I}$ & I I \\
\hline 9 & $L^{\cdot} \varepsilon \angle T$ & $S 90^{\circ} 0$ & $z \angle 0^{\circ} 0$ & $80 \mathrm{~S}^{\circ} \mathrm{I}$ & $\angle S \nabla^{\circ} I$ & Sร9 I & $\nabla 6 S^{\circ} \mathrm{I}$ & $0 \mathrm{I}$ \\
\hline$\varsigma$ & $\varepsilon^{\cdot} 6 L T$ & $890^{\circ} 0$ & $\forall \angle 0^{\circ} 0$ & $60 \mathrm{~S}^{\circ} \mathrm{I}$ & $8 \mathrm{St} \cdot \mathrm{L}$ & St9 $\mathrm{I}$ & $96 \mathrm{~S}^{\circ} \mathrm{T}$ & 6 \\
\hline$t$ & $t^{\circ} 89 \mathrm{I}$ & $\downarrow 90^{\circ} 0$ & $\varepsilon \angle 0^{\circ} 0$ & $0 \mathrm{IS}^{*} \mathrm{I}$ & $09 t^{\circ} \mathrm{I}$ & $\varepsilon$ † $9^{\circ} \mathrm{I}$ & $S 6 S^{\circ} \mathrm{T}$ & 8 \\
\hline $0 \mathrm{I}$ & $Z \cdot 99 I$ & $\angle 90^{\circ} 0$ & $080^{\circ} 0$ & $09 t^{\circ} \mathrm{I}$ & $6 \mathrm{St} \cdot \mathrm{I}$ & $96 \mathrm{~S}^{\circ} \mathrm{I}$ & $96 \mathrm{~S}^{\circ} \mathrm{I}$ & $L$ \\
\hline 8 & $\nabla^{\circ} 28 I$ & $690^{\circ} 0$ & $S \angle 0^{\circ} 0$ & $\angle S V^{\circ} I$ & $8 \mathrm{St} \nabla^{\circ} \mathrm{I}$ & $\angle 6 S^{\circ} I$ & $009^{\circ} \mathrm{I}$ & 9 \\
\hline$\varepsilon$ & $\nabla^{\circ} \angle L T$ & $990^{\circ} 0$ & $2 \angle 0^{\circ} 0$ & $90 S^{*} \mathrm{I}$ & $6 S t^{\circ} \mathrm{T}$ & $8+9^{\circ} \mathrm{T}$ & $\angle 6 S^{\circ} \mathrm{I}$ & $s$ \\
\hline 6 & $\mathrm{I} \cdot \varepsilon 8 \mathrm{I}$ & $690^{\circ} 0$ & $6 \angle 0^{\circ} 0$ & $\operatorname{sst} t^{\circ} \mathrm{I}$ & $\angle S t^{\circ} T$ & $\mathrm{I} 6 \mathrm{~S}^{\circ} \mathrm{I}$ & $269^{\circ} \mathrm{I}$ & $t$ \\
\hline L & $\tau \cdot+8 I$ & $890^{\circ} 0$ & $280^{\circ} 0$ & $\angle S t^{\circ} I$ & $9 S t^{\circ} \mathrm{I}$ & $009^{\circ} \mathrm{I}$ & $S 6 S^{\circ} \mathrm{I}$ & $\varepsilon$ \\
\hline 2 & $\varepsilon \cdot \varepsilon 8 I$ & $690^{\circ} 0$ & $S \angle 0^{\circ} 0$ & $0 \mathrm{IS} \cdot \mathrm{I}$ & $\angle S \nabla^{\circ} I$ & $259^{\circ} \mathrm{I}$ & $\angle 6 \mathrm{~S}^{\circ} \mathrm{I}$ & 2 \\
\hline $\mathrm{I}$ & $\varepsilon \cdot 08 \mathrm{I}$ & $890^{\circ} 0$ & $\angle \angle 0^{\circ} 0$ & $9 S t^{\circ} \mathrm{T}$ & $85 t^{\circ} \mathrm{I}$ & $\mathrm{S} 6 \mathrm{~S}^{\circ} \mathrm{I}$ & $86 \mathrm{~S}^{\circ} \mathrm{I}$ & $\mathrm{I}$ \\
\hline 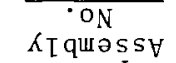 & 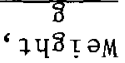 & 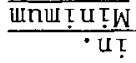 & 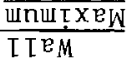 & \multicolumn{2}{|c|}{ 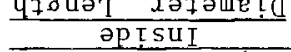 } & \multicolumn{2}{|c|}{$\frac{\partial p I s 7 n_{0}}{\text { suəw } \sigma}$} & $\begin{array}{l}\circ \text { ON } \\
\text { peT: }\end{array}$ \\
\hline
\end{tabular}

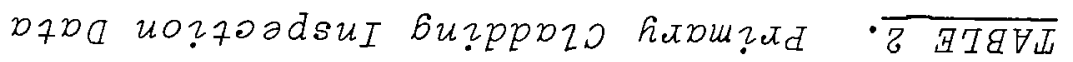




\section{TABLE 3. Secondary CZadding Inspection Data}

\begin{tabular}{|c|c|c|c|c|c|c|c|}
\hline \multirow{2}{*}{$\begin{array}{c}\text { Capsule } \\
\text { As sembly } \\
\text { No. }\end{array}$} & \multicolumn{4}{|c|}{ Dimensions, in. } & \multirow{2}{*}{\multicolumn{2}{|c|}{ Wa11, in. }} & \multirow[b]{2}{*}{ Weight, } \\
\hline & \multicolumn{2}{|c|}{ Outside } & \multicolumn{2}{|c|}{ Inside } & & & \\
\hline 1 & 2.005 & 2.004 & 1.601 & 1.601 & 0.206 & 0.192 & 375.6 \\
\hline 2 * & 2.000 & 2.070 & 1.600 & 1.655 & 0.203 & 0.200 & 524.9 \\
\hline $3 *$ & 2.001 & 2.075 & 1.598 & 1.653 & 0.202 & 0.200 & 528.9 \\
\hline 4 * & 2.000 & 2.072 & 1.596 & 1.658 & 0.210 & 0.200 & 527.2 \\
\hline 5 * & 2.000 & 2.071 & 1.599 & 1.648 & 0.207 & 0.198 & 524.9 \\
\hline $6 *$ & 1.999 & 2.076 & 1.602 & 1.660 & 0.210 & 0.201 & 525.5 \\
\hline 7 & 2.000 & 1.999 & 1.600 & 1.601 & 0.205 & 0.199 & 374.1 \\
\hline 8 & 2.001 & 2.006 & 1.598 & 1.602 & 0.208 & 0.198 & 374.5 \\
\hline 9 & 2.000 & 2.003 & 1.601 & 1.601 & 0.203 & 0.199 & 372.5 \\
\hline 10 & 2.003 & 2.001 & 1.600 & 1.604 & 0.205 & 0.200 & 373.9 \\
\hline 11 & 1.999 & 2.007 & 1.600 & 1.607 & 0.200 & 0.180 & 362.5 \\
\hline 12 & 2.000 & 2.004 & 1.599 & 1.602 & 0.201 & 0.200 & 375.0 \\
\hline 13 & 1.998 & 2.005 & 1.598 & 1.602 & 0.202 & 0.199 & 373.0 \\
\hline 14 & 1.998 & 2.007 & 1.600 & 1.603 & 0.202 & 0.199 & 374.5 \\
\hline 15 & 1.999 & 2.003 & 1.601 & 1.601 & 0.203 & 0.199 & 373.5 \\
\hline 16 & 1.999 & 2.003 & 1.598 & 1.604 & 0.200 & 0.199 & 374.7 \\
\hline 17 & 1.999 & 2.002 & 1.600 & 1.602 & 0.200 & 0.199 & 375.9 \\
\hline 18 & 1.999 & 2.004 & 1.600 & 1.604 & 0.201 & 0.198 & 375.2 \\
\hline 19 & 1.998 & 2.003 & 1.602 & 1.604 & 0.200 & 0.198 & 375.6 \\
\hline 20 & 1.998 & 2.006 & 1.600 & 1.604 & 0.201 & 0.199 & 374.6 \\
\hline 21 & 1.999 & 2.005 & 1.600 & 1.604 & 0.201 & 0.199 & 372.8 \\
\hline 22 & 1.999 & 1.997 & 1.599 & 1.601 & 0.201 & 0.195 & 374.0 \\
\hline 23 & 1.998 & 2.001 & 1.601 & 1.602 & 0.200 & 0.199 & 374.5 \\
\hline 24 & 1.998 & 2.000 & 1.600 & 1.605 & 0.199 & 0.196 & 374.5 \\
\hline 25 & 1.999 & 2.001 & 1.601 & 1.604 & 0.200 & 0.198 & 347.0 \\
\hline 26 & 1.999 & 1.998 & 1.601 & 1.601 & 0.201 & 0.196 & 373.5 \\
\hline 27 & 1.999 & 2.002 & 1.601 & 1.605 & 0.199 & 0.198 & 373.5 \\
\hline 28 & 1.998 & 1.999 & 1.600 & 1.604 & 0.198 & 0.197 & 375.0 \\
\hline 29 & 1.998 & 2.001 & 1.600 & 1.608 & 0.198 & 0.196 & 375.5 \\
\hline 30 & 1.998 & 2.002 & 1.600 & 1.607 & 0.199 & 0.196 & 376.0 \\
\hline 31 & 1.999 & 2.000 & 1.599 & 1.604 & 0.200 & 0.197 & 375.0 \\
\hline 32 & 1.998 & 2.002 & 1.600 & 1.609 & 0.199 & 0.196 & 375.0 \\
\hline 33 & 1.997 & 1.998 & 1.600 & 1.601 & 0.200 & 0.197 & 374.5 \\
\hline 34 & 1.998 & 2.000 & 1.601 & 1.606 & 0.199 & 0.197 & 374.5 \\
\hline 35 & 1.998 & 2.001 & 1.600 & 1.608 & 0.199 & 0.196 & 375.5 \\
\hline 36 & 1.998 & 2.002 & 1.600 & 1.607 & 0.198 & 0.197 & 375.0 \\
\hline 37 & 1.998 & 2.000 & 1.601 & 1.605 & 0.199 & 0.196 & 373.5 \\
\hline 38 & 1.998 & 2.000 & 1.601 & 1.607 & 0.199 & 0.196 & 376.5 \\
\hline 39 & 1.998 & 2.002 & 1.600 & 1.608 & 0.198 & 0.197 & 375.7 \\
\hline 40 & 1.998 & 2.002 & 1.600 & 1.608 & 0.198 & 0.197 & 374.5 \\
\hline
\end{tabular}

* Capsule Assemblies No. 2 through 6 were processed by an alternate High Energy Rate forming process. The final dome ends were machined after HERF. 
TABLE 4. Final Assembly Data on $\mathrm{Sm}_{2} \mathrm{O}_{3}$ Capsules

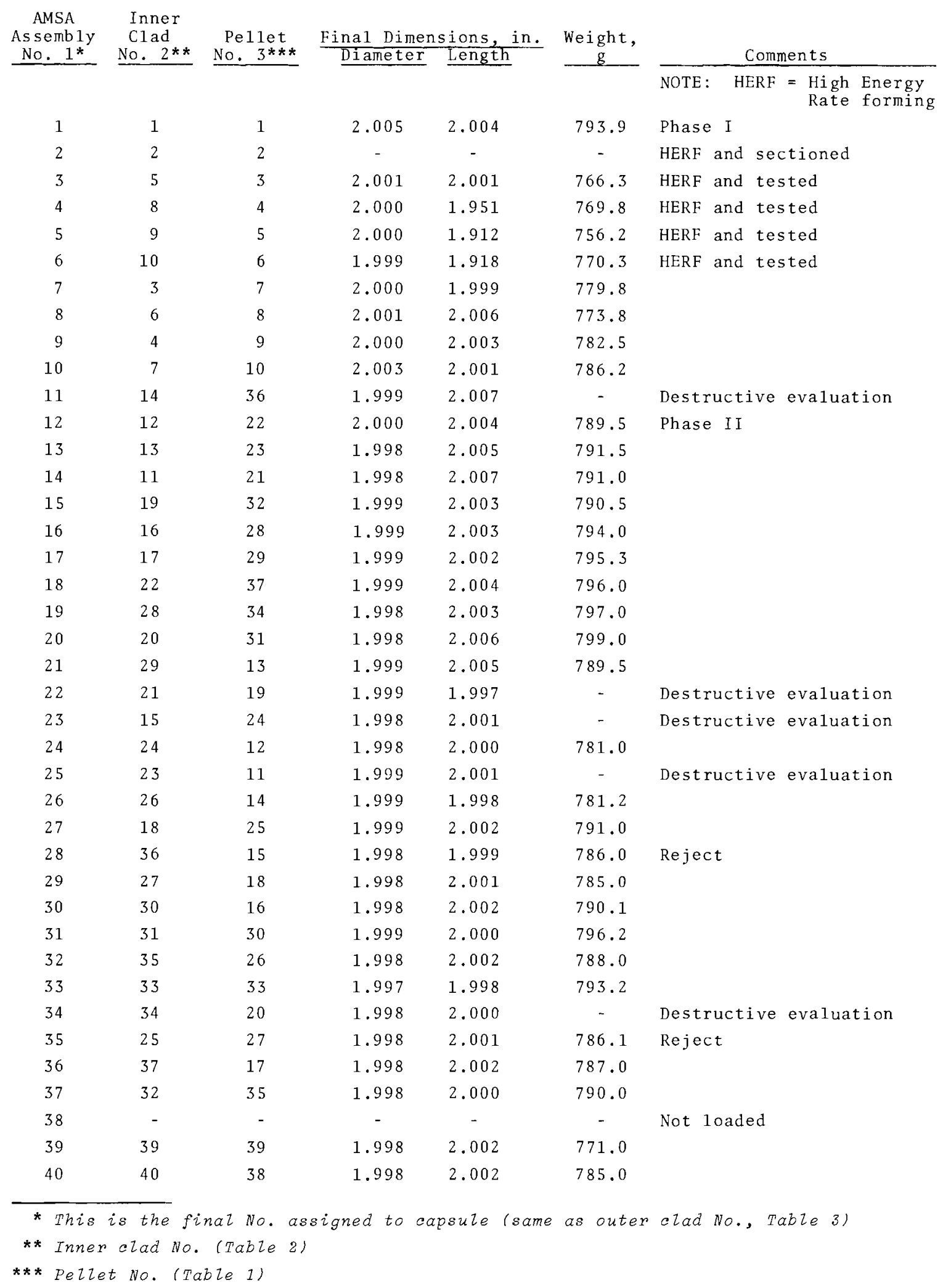


The cladding components were cleaned ultrasonicly using trichlorethylene as a degreaser with a final rinsing in absolute ethyl alcohol. All parts were dried using a hot air gun. Clean, white cotton gloves were used for all subsequent handling steps through final welding. These assembly steps and procedures using $\mathrm{Sm}_{2} \mathrm{O}_{3}$ pellets were developed with particular concern for the potential problems in performing the final radioisotope encapsulation.

A 1 to 2 mil thick tantalum foil liner was inserted around the inside diameter of the inner clad to protect the weld zone after pellet assembly and during welding. This technique, due to the potential for smearable contamination, was critically important.

Inspection points were established and data recorded to maintain quality as well as to correlate process steps with impact test results. In the developmental phase, minor dimensional discrepancies did not necessarily cause rejection, but al1 imperfections were recorded for subsequent process evaluation.

Tantalum and L-605 alloy bar stock were inspected by dye penetrant inspection of discs cut from the ends of the bars. Dye penetrant inspection of machined components was required prior to use to eliminate use of faulty material.

Other inspections during fabrication were used as controls on process development and quality assurance. More than the required number of capsules were fabricated for impact tests so that representative capsules would be available for destructive evaluation throughout the process. These capsules were first examined by a nondestructive, ultrasonic flaw detection technique, and the results were confirmed by metallographic examination of weld areas. Although only a limited number of capsules were sectioned prior to impact testing, all of the 
impacted capsules were sectioned for final evaluation. Excellent correlation existed between nondestructive test results and observed weld microstructures. The high reliability of the ultrasonic test procedures, and the results of impact tests of capsules having known small voids or defects established the welding acceptance criteria for the final phase III ${ }^{147} \mathrm{Pm}_{2} \mathrm{O}_{3}$ capsule.

\section{WELDING DEVELOPMENT}

Development of seal welding by electron beam equipment (Figure 5) may be separated into three major areas:

- Desired penetration,

- Elmination or minimization of defects, and

- Reproducibility.

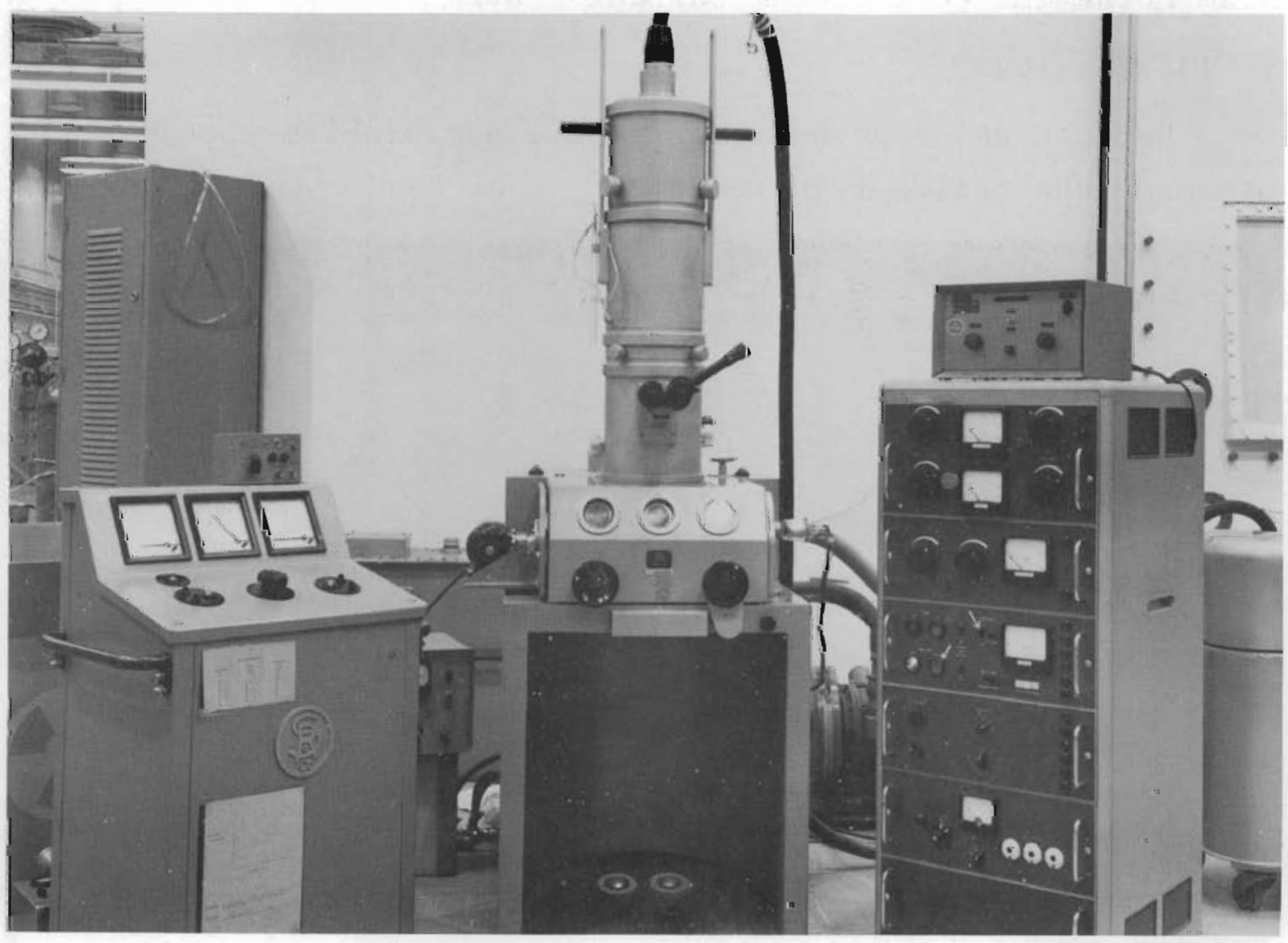

Neg $0662358-3$

EIGURE 5. Electron Beam Welder 
Primary Welds

The seal weld in the tantalum primary cladding is mainly for contamination containment. Release of radioisotopic material during welding is highly undesirable because of equipment contamination, formation of weld inclusions, and degradation of joint integrity. High penetration welds, therefore, could not be used.

Welding experience during Phases I and II of the program indicated that penetration equal to $50 \%$ of the wall thickness was a desirable compromise of the various factors. Parameters for achieving the typical weld shown in Figure 6 were as follows :

Transverse

Beam Potential (kV) Beam Current Beam Deflection Face Speed
135 (no load)
$7.5 \mathrm{~mA}$
$16 \mathrm{mils}$
30 ipm

Defects and reproducibility were not problems because of minimum penetration requirements.

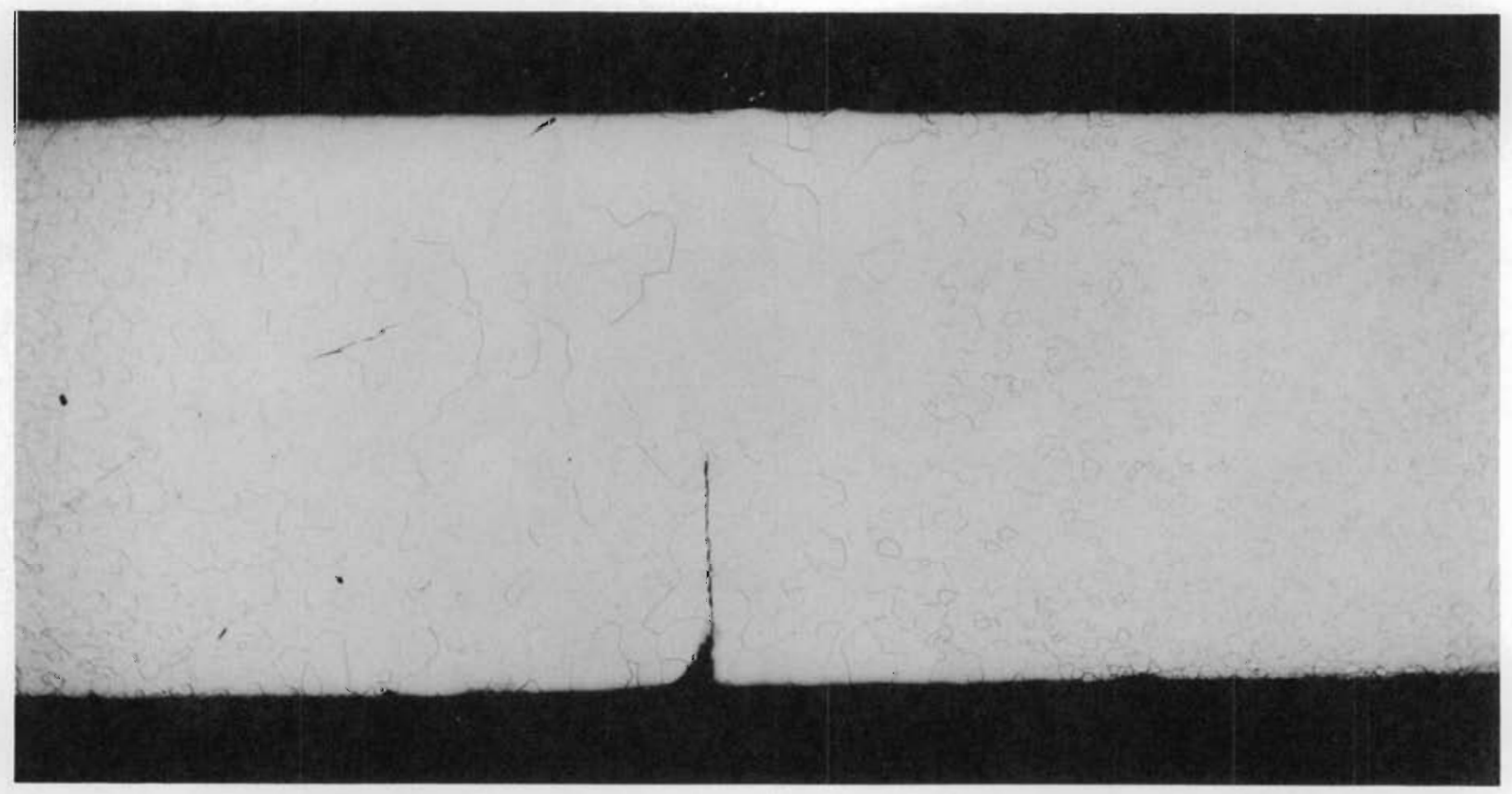

Neg 567-1028

FIGURE 6. Typical mantalum Electron Beam Seal Weld 
Secondary Welds

Initial problems in development of electron beam welds for the L-605 secondary cladding were (1) elimination of excess voids, and (2) reproducible penetration.

Initial test welds were made on joints having a $15 \mathrm{mil}$ step, $100 \mathrm{mils}$ from the inner surface. A 30 mil deflection of the finely focused beam resulted in fully-penetrated welds approximately $43 \mathrm{mils}$ wide.

Postimpaction evaluation of Phase I capsules 1 through 10 (see Table 5) showed three problems:

- Penetration was neither adequate nor consistent.

- Large root voids existed.

- An undesirable "tooth root" weld configuration existed.

A few of the Phase I outer cladding welds exhibited good penetration and an absence of voids. The parameters for these welds, (unintentionally differing from others in beam focus) appeared desirable for subsequent welding. The requisite change in parameters consisted of defocusing the beam by shifting its focal point upward (consequently increasing spot size at the face) and reducing the transverse deflection to 16 mils. Although the new parameter resulted in welds of approximately the same width as those previously made, penetration and weld geometry were perfected and voids were minimized. A typical example of the final weld design utilizing a $15 \mathrm{mil}$ step $15 \mathrm{mils}$ from the inner surface is shown in Figure 7 .

Optimized parameters for Phase II electron beam welding of L-605 a1loy were:

$\frac{\text { Beam Potential (kV) }}{142.5 \text { (no load) }} \frac{\text { Beam Current }}{13.5 \mathrm{~mA}} \frac{\begin{array}{c}\text { Transverse } \\ \text { Beam Deflection }\end{array}}{0.016 \text { in. }} \frac{\text { Face Speed }}{24 \text { ipm }}$ 
BNWL - 994

TABLE 5. Impact Test Data and Postimpaction Evaluation

\begin{tabular}{|c|c|c|c|c|c|c|c|}
\hline $\begin{array}{c}\text { AMSA } \\
\text { Capsule } \\
\text { No. }\end{array}$ & $\begin{array}{l}\text { Impact } \\
\text { Angle }\end{array}$ & $\begin{array}{l}\text { Velocity } \\
\mathrm{ft} / \mathrm{sec} \\
\end{array}$ & & $\begin{array}{l}\text { arget } \\
\text { terial }\end{array}$ & Results & $\begin{array}{r}\text { Post } \\
\text { We1d Inspec } \\
\end{array}$ & $\begin{array}{l}\text { timpaction } \\
\text { ction and comments }\end{array}$ \\
\hline 1 & $22-1 / 2$ & 414 & LV & granite & intact & $90 \%$ pent: - & - excellent we $1 \mathrm{~d}$ \\
\hline 2 & not tested & - & & - & - & Destructive & e evaluation \\
\hline 3 & $22-1 / 2$ & 400 & ES & granite & intact & $80 \%$ pent. - & - weld root voids \\
\hline 4 & 45 & 396 & LV & granite & intact & $45 \%$ pent. & - weld reject \\
\hline 5 & 0 & 396 & ES & granite & intact & $95 \%$ pent. & - excellent weld \\
\hline 6 & $67-1 / 2$ & 398 & ES & granite & intact & $95 \%$ pent. & - excellent weld \\
\hline 7 & 45 & 406 & ES & granite & rupture & $50 \%$ pent. & - reject \\
\hline 8 & 0 & 403 & ES & granite & intact & $70 \%$ pent. & - reject \\
\hline 9 & $67-1 / 2$ & 407 & ES & granite & intact & $40 \%$ pent. & - reject \\
\hline 10 & 90 & 410 & ES & granite & intact & $75 \%$ pent. & - weld root voids \\
\hline 11 & not tested & - & & - & - & Destructive & e evaluation \\
\hline 12 & 0 & 494 & ES & granite & intact & $90 \%$ pent. - & excellent weld \\
\hline 13 & $22-1 / 2$ & 494 & ES & granite & rupture & $90 \%$ pent. & - excellent weld \\
\hline 14 & 45 & 479 & ES & granite & rupture & $85 \%$ pent. & - excellent weld \\
\hline 15 & $67-1 / 2$ & 479 & ES & granite & intact & $95 \%$ pent. & - excellent weld \\
\hline 16 & 0 & 485 & ES & granite & intact & $95 \%$ pent. & - small voids \\
\hline 17 & $22-1 / 2$ & 485 & ES & granite & rupture & $95 \%$ pent. & - excellent weld \\
\hline 18 & 45 & 487 & ES & granite & rupture & $95 \%$ pent. & - smal1 voids \\
\hline 19 & 45 & 381 & ES & granite & rupture & $95 \%$ pent. & - excellent weld \\
\hline 20 & 90 & 487 & ES & granite & intact & $95 \%$ pent. & $-\sim 0.030 \mathrm{in.}$ void \\
\hline 21 & 45 & 329 & ES & granite & intact & $95 \%$ pent. - & - excellent weld \\
\hline 22 & not tested & - & & - & - & Destructive & evaluation \\
\hline 23 & not tested & - & & - & - & Destructive & evaluation \\
\hline 24 & $22-1 / 2$ & 429 & ES & granite & intact & $90 \%$ pent. - & excellent weld \\
\hline 25 & not tested & - & & - & - & Destructive & evaluation \\
\hline 26 & 35 & 388 & Es & granite & rupture & $90 \%$ pent. - & $\begin{array}{l}-0.010-0.015 \text { in. } \\
\text { voids }\end{array}$ \\
\hline 27 & 45 & 429 & ES & granite & rupture & pent. & - excellent we $1 d$ \\
\hline 28 & $\begin{array}{l}30 \\
45\end{array}$ & $\begin{array}{l}630 \\
285\end{array}$ & ES & $\begin{array}{l}\text { soil } \\
\text { granite }\end{array}$ & $\begin{array}{l}\text { intact } \\
\text { rupture }\end{array}$ & $95 \%$ pent & - excellent weld \\
\hline 29 & 55 & 388 & ES & granite & rupture & $85 \%$ pent. & - excellent weld \\
\hline 30 & 35 & 374 & ES & granite & intact & $95 \%$ pent. & - excellent weld \\
\hline 31 & 45 & 374 & ES & granite & rupture & $95 \%$ pent. & - excellent weld \\
\hline 32 & 45 & 323 & ES & granite & rupture & $85 \%$ pent. & $\begin{array}{l}0.010-0.015 \text { in. } \\
\text { voids }\end{array}$ \\
\hline 33 & 45 & 392 & & ncrete & intact & $90 \%$ pent. - & excellent weld \\
\hline 34 & not testec & - & & - & - & Destructive & evaluation \\
\hline 35 & not tested & - & & - & - & Wind tunne 1 & test - WPAFB \\
\hline 36 & 45 & 312 & ES & granite & rupture & 95\% pent. - & excellent weld \\
\hline 37 & 45 & 285 & ES & granite & intact & $95 \%$ pent. - & -0.030 in. void \\
\hline 38 & - & - & & - & - & Not loaded & \\
\hline 39 & 45 & 678 & & ncrete & intact & $85 \%$ pent: - & - sma11 voids \\
\hline 40 & 45 & 650 & & ncrete & intact & Not section & ned \\
\hline
\end{tabular}


BNWL - 994

\section{TABLE 5. (contd)}

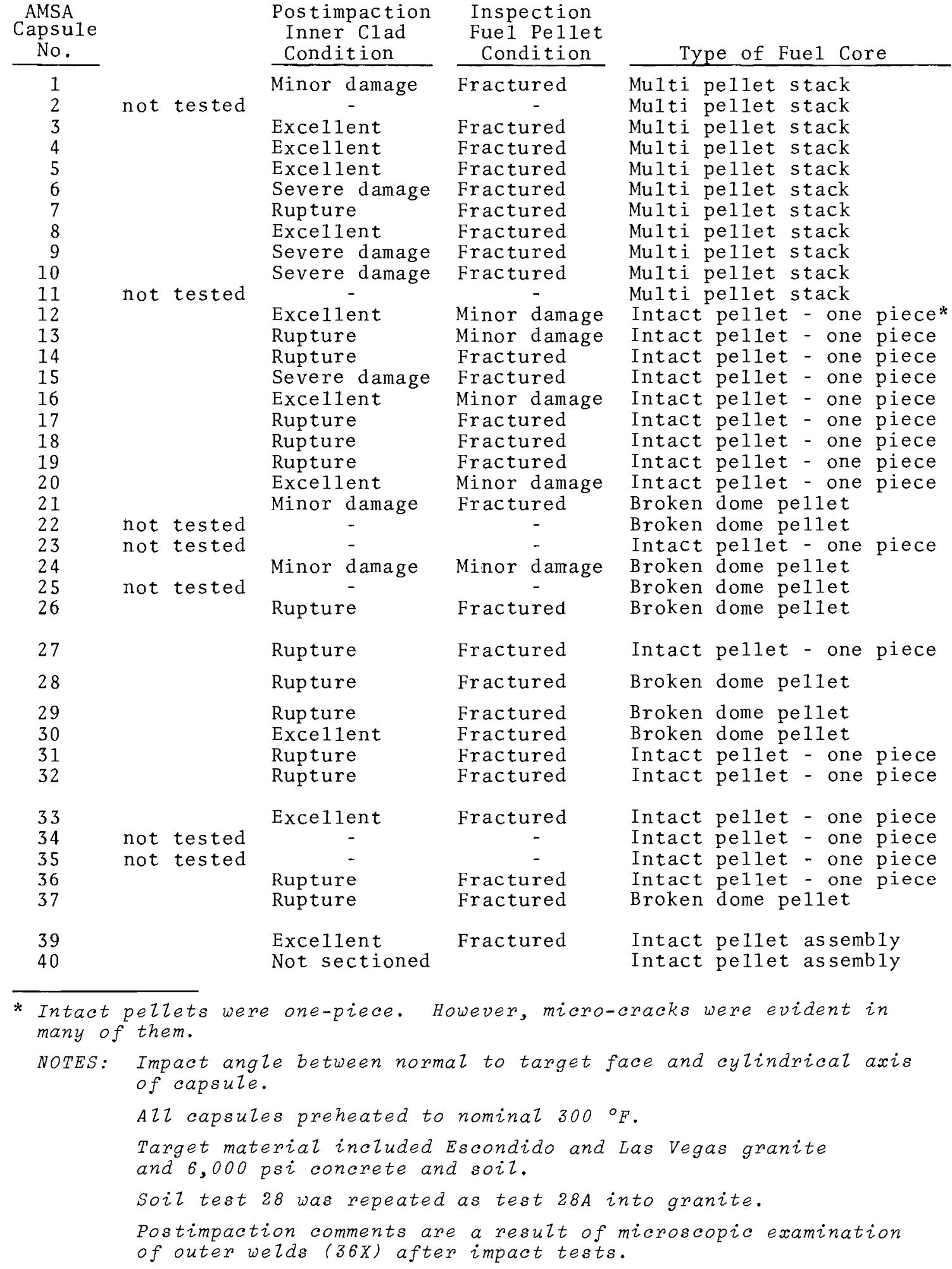


BNWL - 994

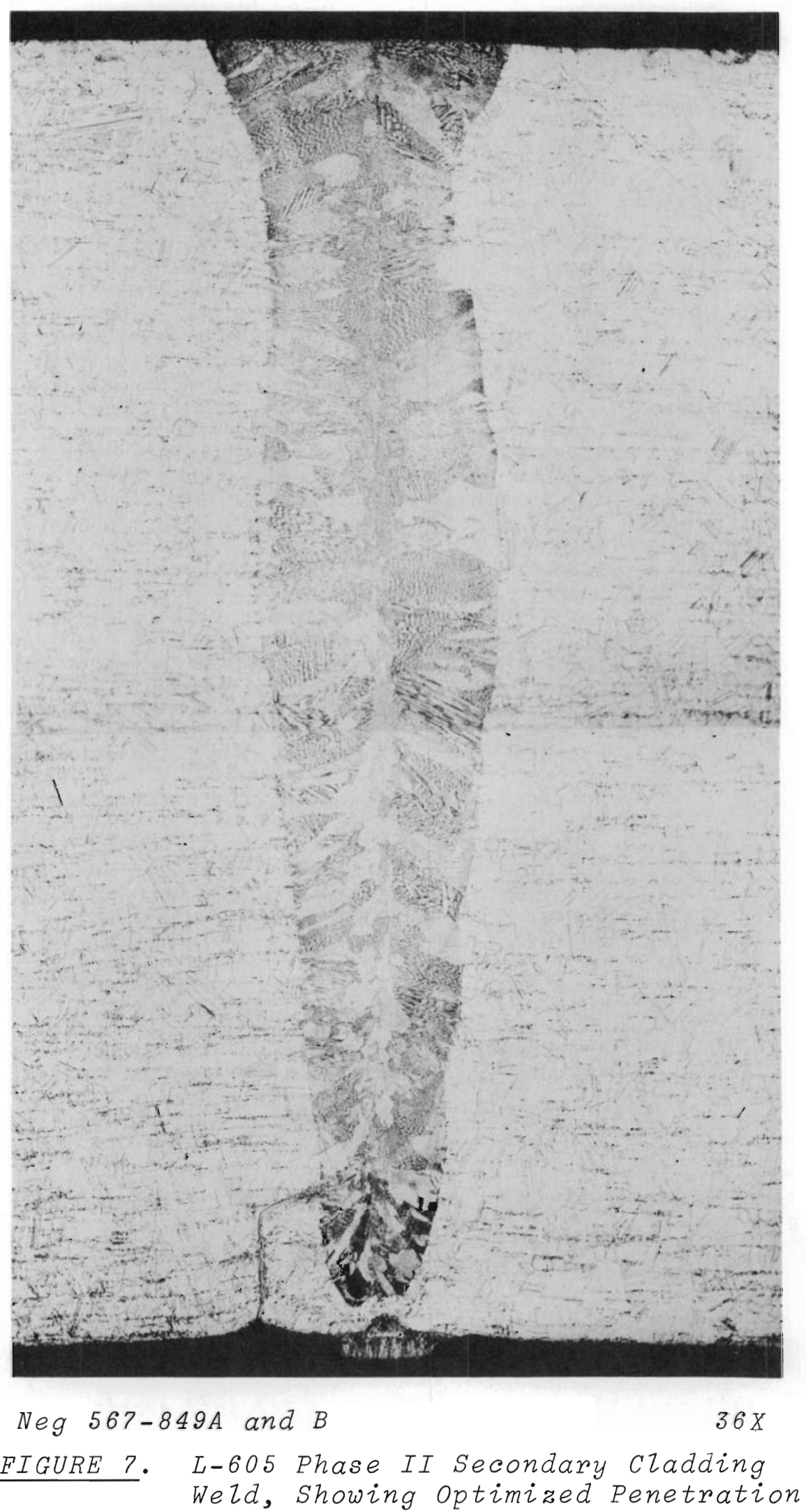




\section{ALTERNATE PROCESS DEVELOPMENT}

Capsules $2,3,4,5$, and 6 were fabricated by an alternate process using high-energy rate forming (HERF). This process appeared to have potential for improving impact resistance. The pressed and sintered cores were seal-welded into a doubleclad tantalum and L-605 billet. The billet was then heated to $1100{ }^{\circ} \mathrm{C}$ and consolidated by HERF using pressures in excess of 200,000 psi. The HERF punch and die components are shown in Figure 8. The HERF consolidation process effected intimate interfacial contact between the billet components and increased the billet diameter slightly (Figure 9). Because of this increase in diameter, it was necessary to remove as much as 0.025 in. to $0.030 \mathrm{in}$. from the outer cladding thickness

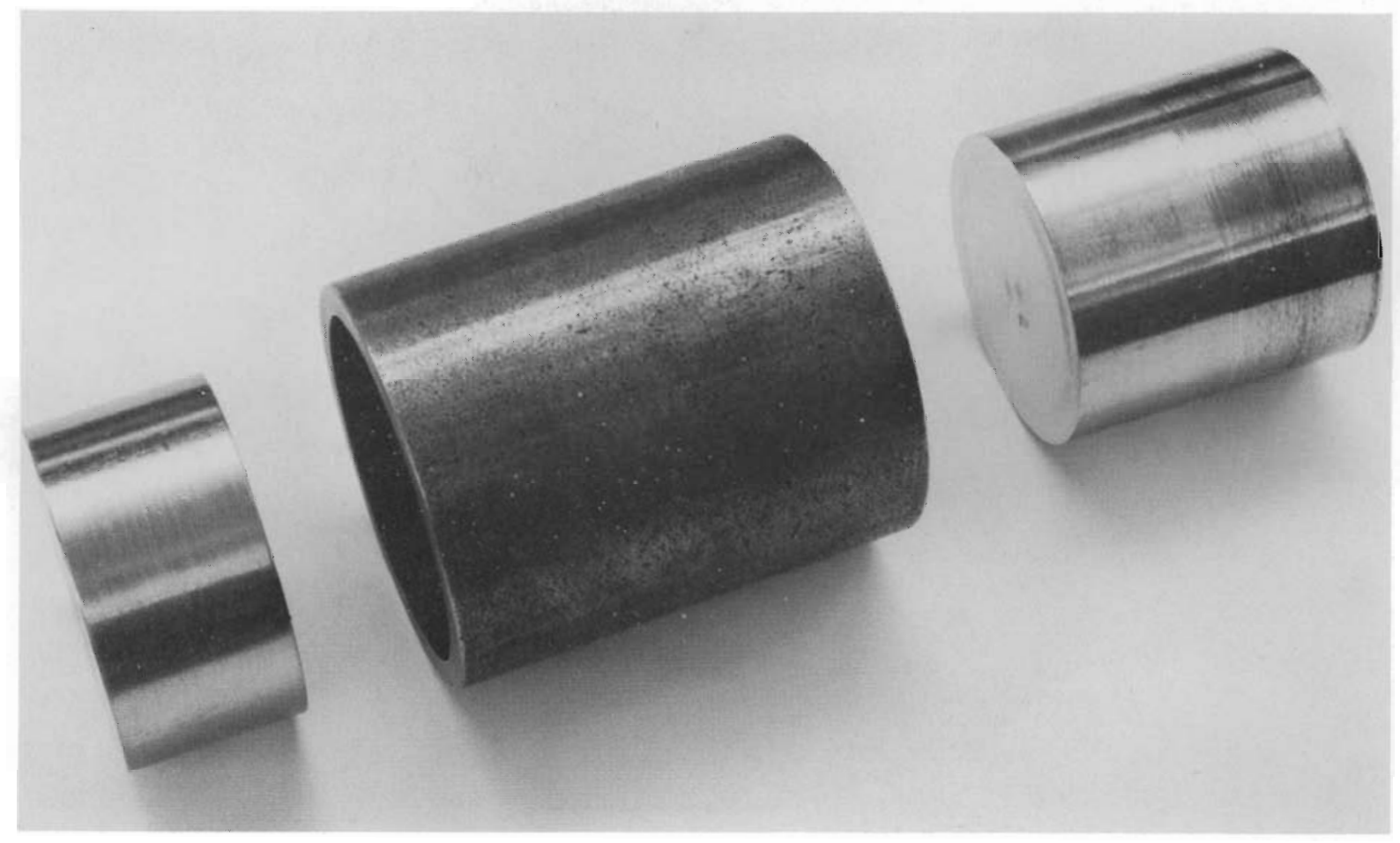

Neg 0670660-4

FIGURE 8. HERF Die and Punches 
during final machining. This loss of wall thickness apparently did not degrade the impact resistance, since these capsules did not rupture during impact testing (Table 5). Although the HERF process could probably have been perfected to yield as-formed shapes to more exacting dimensions, fewer process variables were involved in the slip-fit and weld assembly process. The program time schedule dictated adoption of the simpler approach. The slip-fit system was considered completely adequate for this isotope application.

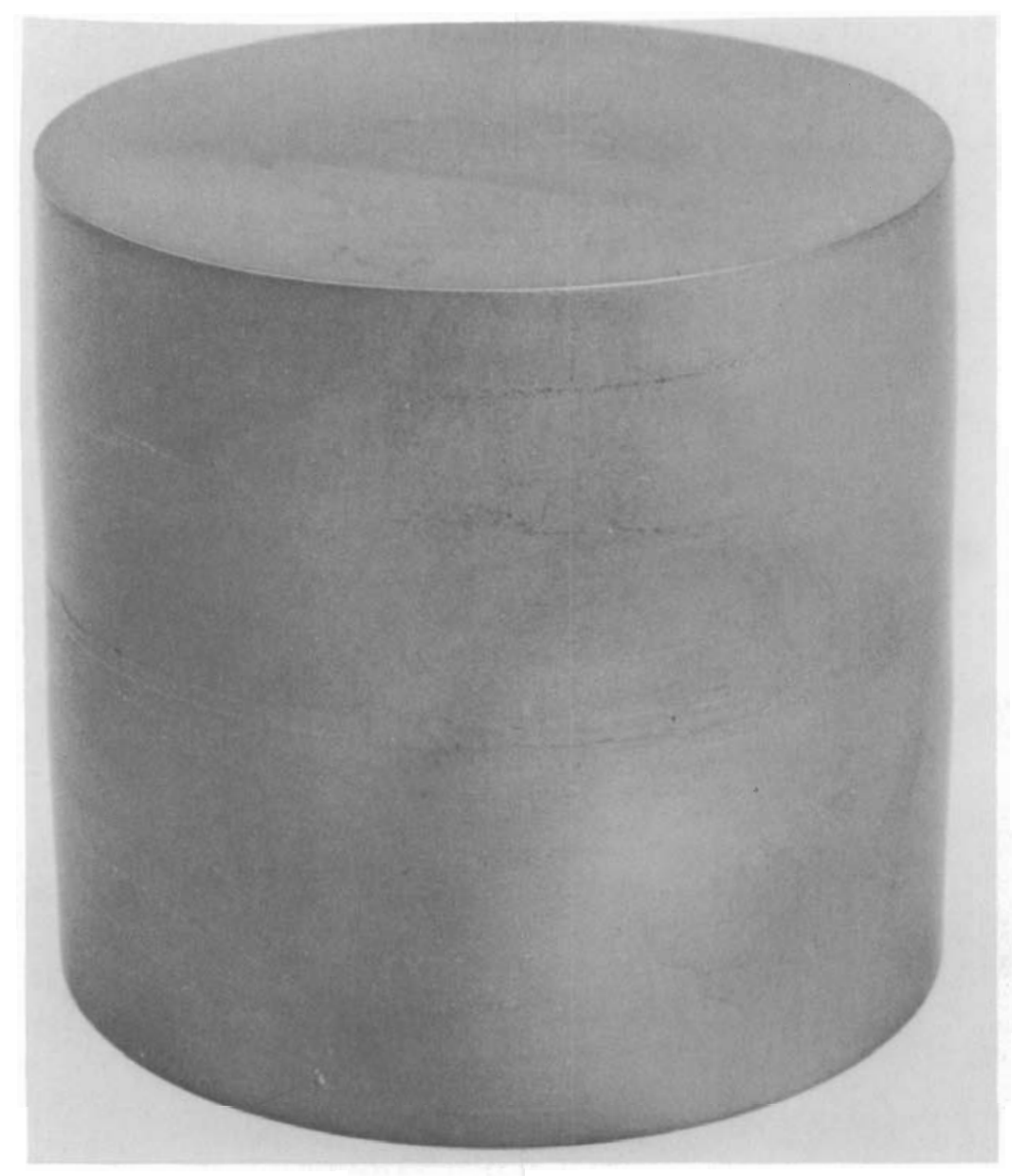

Neg $0670692-2$

FIGURE 9. Double Clad Capsule High-Energy Rate Formed, Ready for Final Macinining 


\section{QUALIFICATION CAPSULES PHASE II}

The Phase II capsule qualifications for this program overlapped considerably into the final evaluation of the design and development Phase I. The basic design established at the beginning of the program did not change. Program time schedules did not allow major changes or experimentation, however, the only questionable areas after the completion of Phase I were process welding problems and improved detection of defects by nondestructive testing methods.

By means of an extensive review of postimpaction weld inspections (Table 5), the nondestructive ultrasonic test was refined to identify both the degree of weld penetration and voids as smal1 as 0.010 in. in size.

The process flow was revised to delete the alternate HERF process and to add the nondestructive testing inspections. With this plan in effect, the qualification Phase II capsules were fabricated. The same lot of samarium was used for producing all core pellets (Table 1) and the same heat of material was used for all of the cladding components (Tables 2 and 3 ). The chemical analyses of these materials are included in the detailed quality control summary of the fueled capsule in the Appendix of this report.

\section{IMPACT TESTS AND POSTIMPACTION EVALUATION}

Table 5 (Impact Test Data and Postimpaction Evaluation) provides an overall summary of the individual tests. This table provides information regarding impact conditions, postimpaction inspection, and fabrication history for every capsule. The AMSA capsule number is a control number that can be referenced to previous Tables 1 through 4 for pellet, inner and outer cladding, and assembly data. 
Details of the impact testing are covered in W. J. Dalby's Sandia impact test report. (1) Fuel containment except in the most adverse impact conditions, can be expected. A typical example of an impact failure is shown in Figures 10 and 11 . When impacted against a granite target at, or near the critical impact angle $\left(45^{\circ}\right.$ from the cylindrical axis of the capsule), and at a velocity exceeding $80 \%$ of terminal velocity, a capsule may be expected to fail. These conditions involve the extremely hard (40,000 psi compressive strength) Escondido granite. The impact deformed surface area increases as the impact attitude is shifted in either direction from the critical $45^{\circ}$ position. The increased surface area results in a reduced impaction energy absorption per unit area.

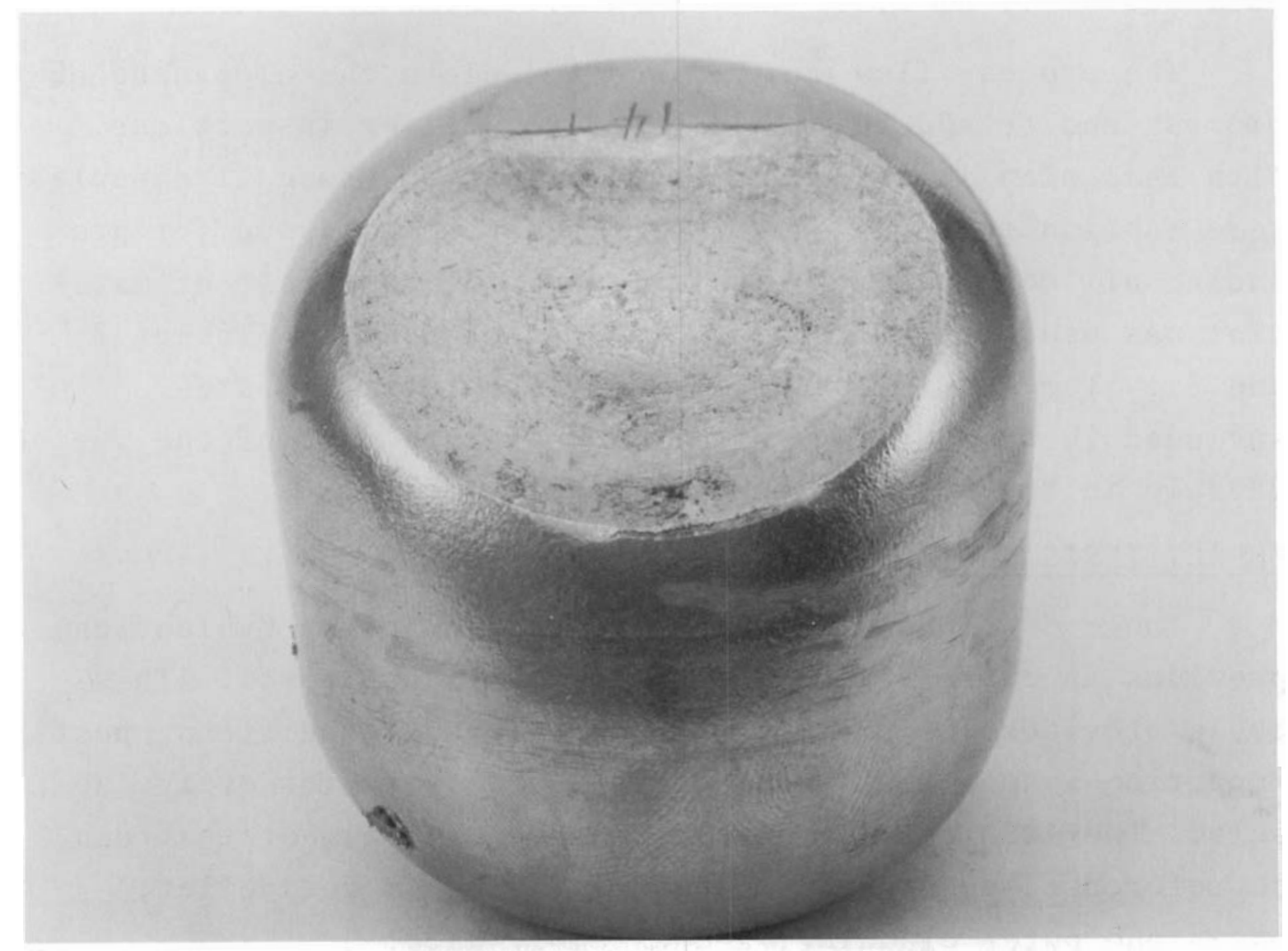

Neg $0671777-25$

FIGURE 10. $45^{\circ}$ Impact Faizure 


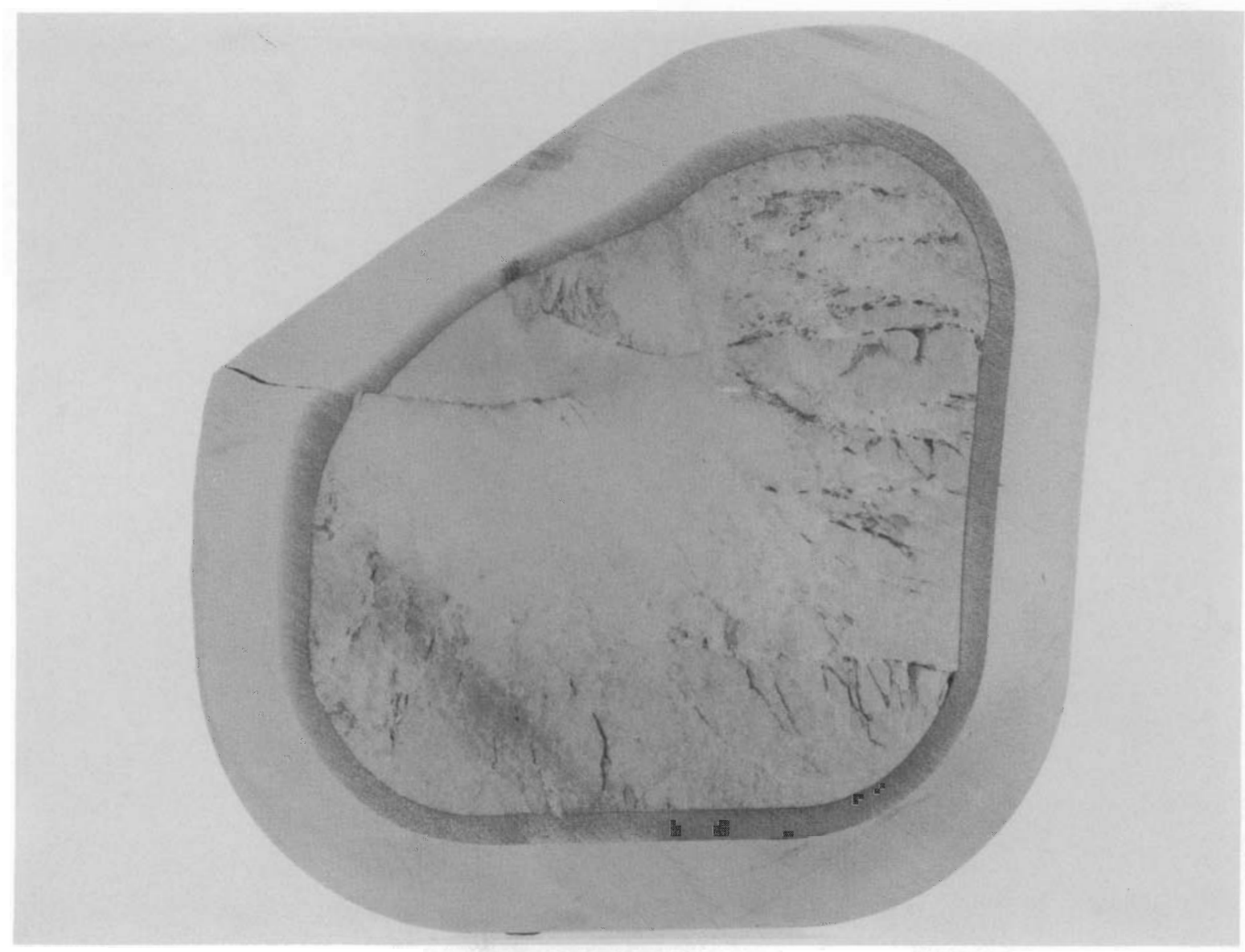

Neg $0671797-37$

FIGURE 11. $45^{\circ}$ Impact Failure as sectioned

The performance of the capsule against 6,000 psi concrete and soil targets at the critical impact angle (Table 5) presents an entirely different set of test results. These capsules remain intact at velocities as high as $678 \mathrm{ft} / \mathrm{sec}$ or approximately twice the terminal velocity. A capsule after impact at this velocity against 6,000 psi concrete is shown in Figure 12 . 
BNWL-994

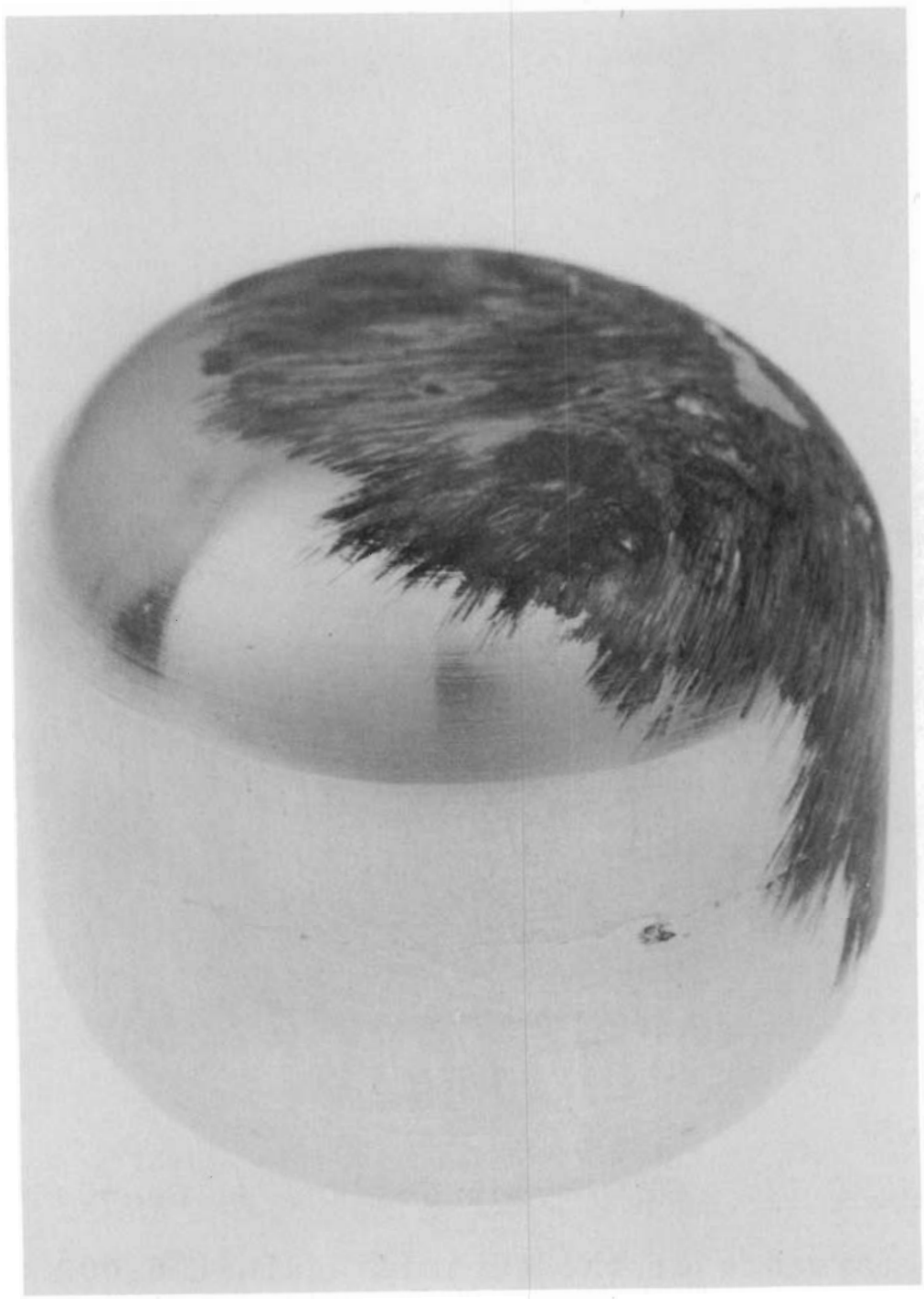

Neg 0683430-1

EIGURE 12. AMSA Capsule After Impact Against Concrete at $678 \mathrm{ft} / \mathrm{sec}$ 


\section{AMSA HEAT SOURCE FABRICATION PHASE III}

At the completion of the impact qualification testing evaluation, a complete review of the process technology and quality control requirements was made. A AMSA radioisotope encapsulation, process description, and quality control plan was prepared (Figure 13).

Process fabrication steps were frozen. All the pertinent quality control data covering test results of the 14 types of inspections indicated on the flow chart (Figure 13) are exhibited either in the following test or accumulated in the appendix.

\section{PROMETHIUM PURIFICATION}

The rare earth elements resulting from nuclear fission and decay are isolated from Hanford Purex Plant waste by utilizing a series of successive precipitation processes. $(2,3)$ After storage to allow for decay of the gamma emitting ${ }^{148} \mathrm{Pm}$, the rare earth crude cut is further refined by solvent extraction, (3) thermal concentration, and "acid ki11"(4) steps prior to transfer to the Pacific Northwest Laboratory's Hot-Ce11 Facility.

Isolation of promethium from its adjacent rare earth neighbors and from all other impurities is accomplished at BNW by a displacement cation exchange process ${ }^{(5)}$ utilizing an ammonia buffered solution of the complexing agent DTPA* as the separating agent. The separation is conducted in a seven-column ion-exchange facility installed, together with associated tanks, valves, and instrumentation, in a heavily shielded remote1y operated ce11.

With the very high ${ }^{144} \mathrm{Ce}$ and ${ }^{154} \mathrm{Eu}$ separation factors achieved in the ion-exchange process, purified promethium does not require extensive shielding from the final conversion

* [(Carboxymethyzimino)bis (ethyzenenitrizo)] tetra-acetic acid. 
BNWL-994

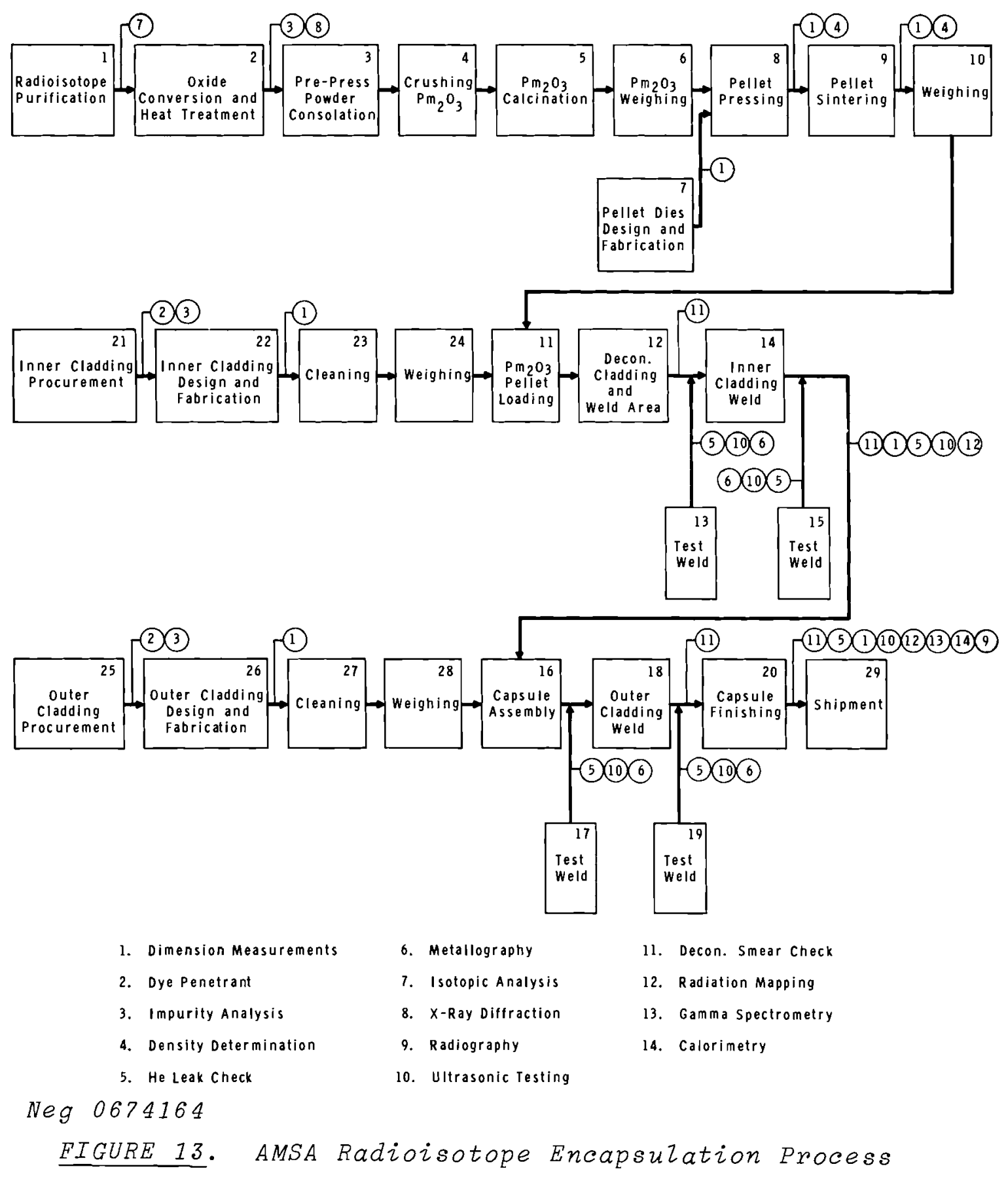


to the sesquioxide. The promethium product solution, collected as it flows from the final purification column, is transferred to the lightly shielded glove box facilities for oxide conversion to the oxide.

Radiochemical analysis, corrected to November 3, 1967, indicated the AMSA ${ }^{147} \mathrm{Pm}_{2} \mathrm{O}_{3}$ capsule to contain:

- $\quad 147 \mathrm{Pm}: 168,200 \mathrm{Ci}$

- ${ }^{146} \mathrm{Pm}: 0.25 \pm 0.2 \times 10^{-6} \mathrm{Ci} / \mathrm{Ci}{ }^{147} \mathrm{Pm}$ or $0.042 \mathrm{Ci}$

- ${ }^{148} \mathrm{Pm}: \mathrm{Ci}{ }^{148} \mathrm{Pm} / \mathrm{Ci}{ }^{146} \mathrm{Pm} \leq 0.04$ or $<0.002 \mathrm{Ci}$

- ${ }^{154} \mathrm{Eu}: 3 \times 10^{-9} \mathrm{Ci} / \mathrm{Ci}{ }^{147} \mathrm{Pm}$ or $5 \times 10^{-4} \mathrm{Ci}$

- ${ }^{144} \mathrm{Ce}:<10^{-9} \mathrm{Ci} / \mathrm{Ci}{ }^{147} \mathrm{Pm}$ or $<2 \times 10^{-4} \mathrm{Ci}$

The ${ }^{147}$ Sm daughter, although constituting the principal impurity, was less than $1 \%$ on the date of promethium purification. Non-rare earth impurities spectrographically detected were $0.02 \% \mathrm{~A} 1,<0.001 \% \mathrm{Fe}, 0.005 \% \mathrm{Mg}$, and $<0.005 \% \mathrm{Si}$ and, in aggregate, constituted less than $1 \%$ of the oxide. X-ray analysis of the treated oxide confirmed the crystal structure to be monoclinic.

\section{POWDER PREPARATION}

The purified ${ }^{147} \mathrm{Pm}_{2} \mathrm{O}_{3}$, after the conversion and heat treatment, is difficult to control due to its extremely small particle size (approximately 1 micron). This physical characteristic of the promethium powder, as with the stand-in $\mathrm{Sm}_{2} \mathrm{O}_{3}$, makes it difficult to press to the optimum density for sintering. In the case of ${ }^{147} \mathrm{Pm}_{2} \mathrm{O}_{3}$, the radiation exposure to glove box operators increases with spread of the highly mobile ${ }^{147} \mathrm{Pm}_{2} \mathrm{O}_{3}$ powder around the glove box. The micronized powder can thus create a difficult exposure problem. This problem is reduced when the powder is consolidated by a prepressing (slugging) operation. Upon crumbling this material to a specified particle size range, a relatively dustless, free-flowing powder is obtained which may then be pressed a 
second time into the shape and volume required to produce the final sintered density needed for assembly.

The parameters to produce the most favorable powder load for the AMSA prepressing die appeared to be 25 tons/in. ${ }^{2}$ (tsi) in increments of 35 to $40 \mathrm{~g}$ of ${ }^{147} \mathrm{Pm}_{2} \mathrm{O}_{3}$. This would produce a density of 55 to $60 \%$ TD dependent on the increment powder load. These pellets were crushed and screened through a 12 mesh stainless steel screen to produce a granular powder with a tap density of approximately $30 \% \mathrm{TD}$.

A special calibrated powder container was fabricated to transfer the ${ }^{147} \mathrm{Pm}_{2} \mathrm{O}_{3}$ powder from the screening step to the die loading operation. This container (Figure 14) had a volume sufficient to contain the required powder for a die load. By means of the slide release at the bottom of this container, the die could be loaded with a minimum of powder loss, thus reducing the spread of ${ }^{147} \mathrm{Pm}$ throughout the glove box.

\section{PRESSING AND SINTERING}

The die design used for the stand-in $\mathrm{Sm}_{2} \mathrm{O}_{3}$ pellet fabrication was modified for use in the pressing of the promethium pellets. This punch and die set (Figure 15), utilizing a tapered and split sleeve, was very successful in producing highly dense $\mathrm{Sm}_{2} \mathrm{O}_{3}$ pellets for the impact test capsules.

An increase in die diameter was predicted on the bas is of earlier experience indicating a lower pressed density and a greater radial reduction during the sintering of promethium compared to samarium. The average diameter reduction with $\mathrm{Sm}_{2} \mathrm{O}_{3}$ during the present program was approximately $7 \%$, while the length reduction was slightly greater than $5 \%$. These data, summarized and reproduced from Part $I$ of this report, are shown in Table 6. Previous experience with ${ }^{147} \mathrm{Pm}_{2} \mathrm{O}_{3}$ pellets 


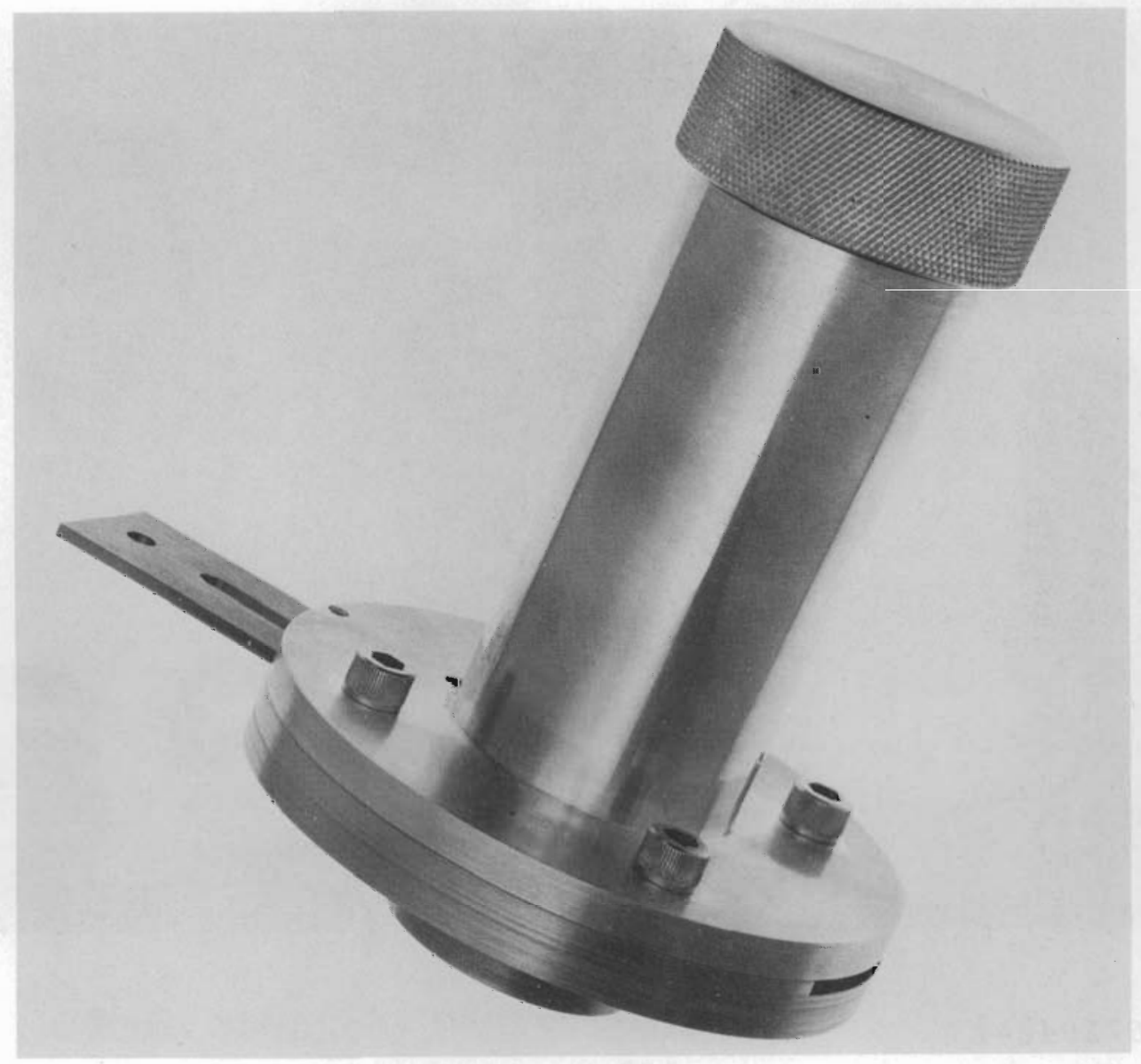

\section{Neg $0672551-1$}

FIGURE 14. $\quad{ }^{147} \mathrm{Pm}_{2} \mathrm{O}_{3}$ Powder Container

having diameters $25 \%$ less than the AMSA pellet and lengths of 0.25 in. showed diameter reductions of $9 \%$ and length reductions on the order of $7 \%$.

By correlating this experience with the $\mathrm{Sm}_{2} \mathrm{O}_{3}$ sintering data from the present program, it appeared that greater shrinkage would be experienced with the ${ }^{147} \mathrm{Pm}_{2} \mathrm{O}_{3}$ AMSA pe11et during sintering. Since the $\mathrm{Sm}_{2} \mathrm{O}_{3}$ AMSA pellets were assembled in the inner cladding, with extra tolerance on the diameter and minimum clearance axially, a decision was made to increase the die sleeve diameter. 


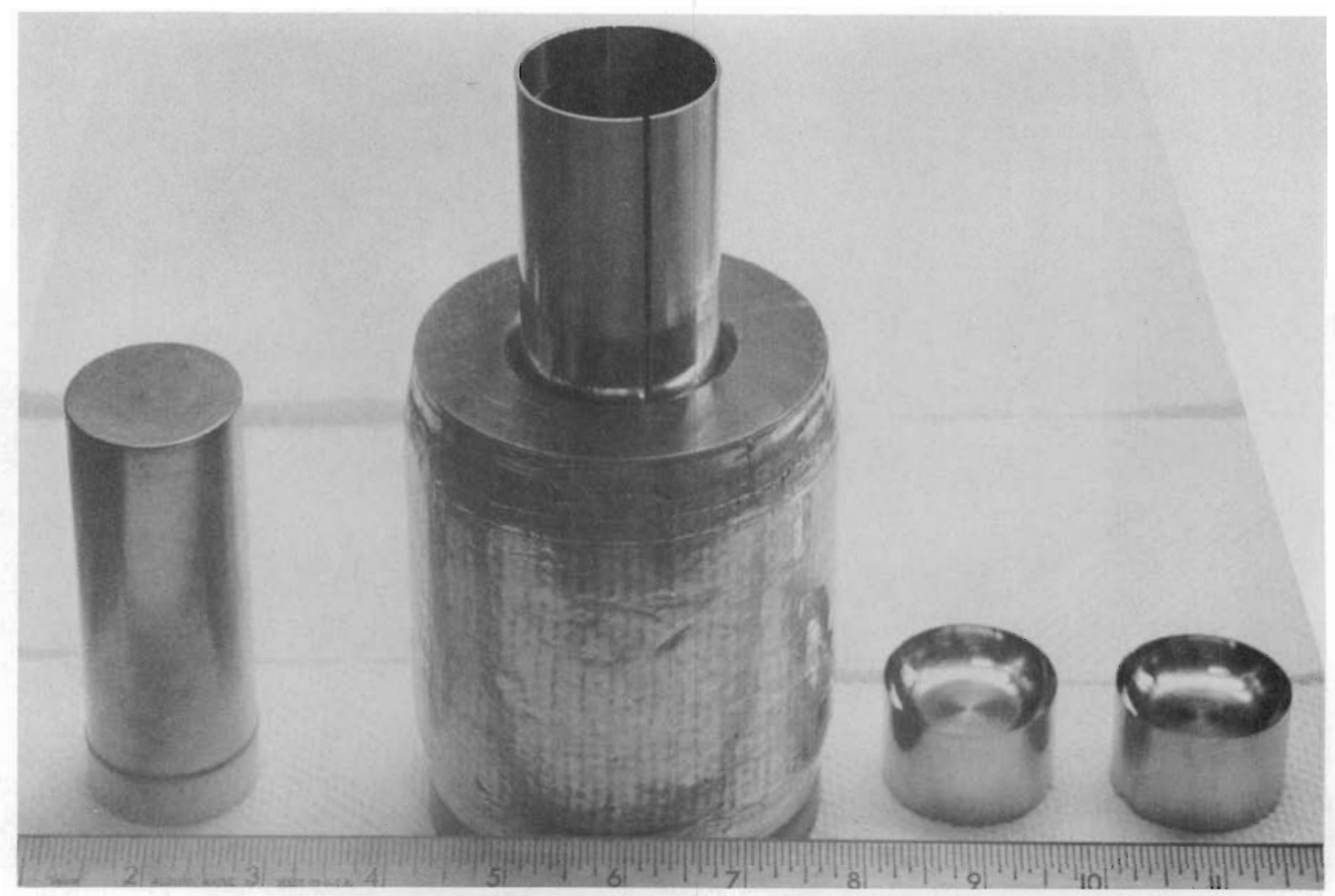

Neg $0672642-1$

FIGURE 15. $\quad{ }^{147} \mathrm{Pm}_{2} \mathrm{O}_{3}$ Modified Die TABLE 6. $\mathrm{Sm}_{2} \mathrm{O}_{3}$ Pellets - AMSA Phase II

Pressing Data

\begin{tabular}{|c|c|c|c|}
\hline Run* & Pressure, & \multicolumn{2}{|c|}{ Dimensions } \\
\hline No. & tsi & Diam & Length \\
\hline $\mathrm{R}-1$ & 64.5 & 1.550 & 1.526 \\
\hline $\mathrm{R}-2$ & 64.5 & 1.550 & 1.524 \\
\hline $\mathrm{R}-3$ & 64.5 & 1.550 & 1.524 \\
\hline$R-4$ & 64.5 & 1.552 & 1.523 \\
\hline
\end{tabular}

Sintering Data

\begin{tabular}{|c|c|c|c|c|c|}
\hline Time, & Temp., & Dime & ions & \multicolumn{2}{|c|}{ Reduction, : } \\
\hline $\mathrm{hr}$ & ${ }^{\circ} \mathrm{C}$ & Diam & Length & Diam & Length \\
\hline 6 & 1350 & 1.450 & 1.450 & 6.4 & 4.9 \\
\hline 9 & 1350 & 1.440 & 1.445 & 7.1 & 5.2 \\
\hline 21 & 1350 & 1.440 & 1.445 & 7.1 & 5.2 \\
\hline 14 & 1400 & 1.433 & 1.430 & 7.7 & 6.1 \\
\hline
\end{tabular}

* Run No. represents average dimension of six pelzets per run. Diameter variation $= \pm 0.001 \mathrm{in}$. Length variation $= \pm 0.010 \mathrm{in}$.

Run $R-4$ - porosity determination

As pressed $75 \%$ TD

As sintered $95 \%$ TD 
The die was accordingly modified by increasing the internal diameter by 0.020 in. The back-up plugs, to produce the dome shaped ends, were remade to fit the new die bore.

After the die was modified to the larger internal diameter, three test runs were made using typical pressing conditions. The 200 ton press was attached by use of an extension from the shielded gloved enclosure (Figure 16). $\mathrm{Sm}_{2} \mathrm{O}_{3}$ was prepressed, screened, and loaded into the die by use of the special powder container. The results of these experiments are tabulated as follows:

\begin{tabular}{|c|c|c|c|c|c|c|c|c|c|}
\hline \multicolumn{4}{|c|}{ Pressing Data } & \multicolumn{6}{|c|}{ Sintering Data } \\
\hline Pellet & Pressure, & Dimens & ons, in. & Time, & Temp., & Dimen & ons, in & Redu & on, : $\frac{\circ}{6}$ \\
\hline No. & $t s i$ & diam & length & $\mathrm{hr}$ & & diam & length & diam & Iength \\
\hline SM- 1 & 63 & 1.570 & 1.477 & 12 & 1450 & 1.443 & 1.400 & 8.0 & 5.2 \\
\hline SM-2 & 58 & 1.570 & 1.483 & 12 & 1450 & 1.448 & 1.425 & 7.8 & 3.9 \\
\hline SM- 3 & 53 & 1.570 & 1.507 & 12 & 1450 & 1.440 & 1.440 & 8.2 & 4.4 \\
\hline
\end{tabular}

A11 three of these pellets were sintered within the heat zone in a single furnace run (Figure 17). Although microcracks were noted at the transition point where the straight cylinder blended into the radii for the dome ends, the pellets were intact and sintered reductions were suitable for assembly. These pellets were used for test weld capsules. These pellets, comparable to the previous Phase II pellets, showed a slightly greater radial reduction and a slightly smaller length reduction. Although the applied pressure should have a direct relationship to the final reduction during sintering, this effect was probably obscured by thermal gradients within the furnace. The center position in the furnace probably accounts for the better sintering results in the SM-1 pellet.

The first attempt to press ${ }^{147} \mathrm{Pm}_{2} \mathrm{O}_{3}$ as a single, onepiece pellet failed. To duplicate the optimum-process parameters developed with the $\mathrm{Sm}_{2} \mathrm{O}_{3}$ powder, the die was assembled with the required quantity of powder and 63 tsi was 
BNWL-994

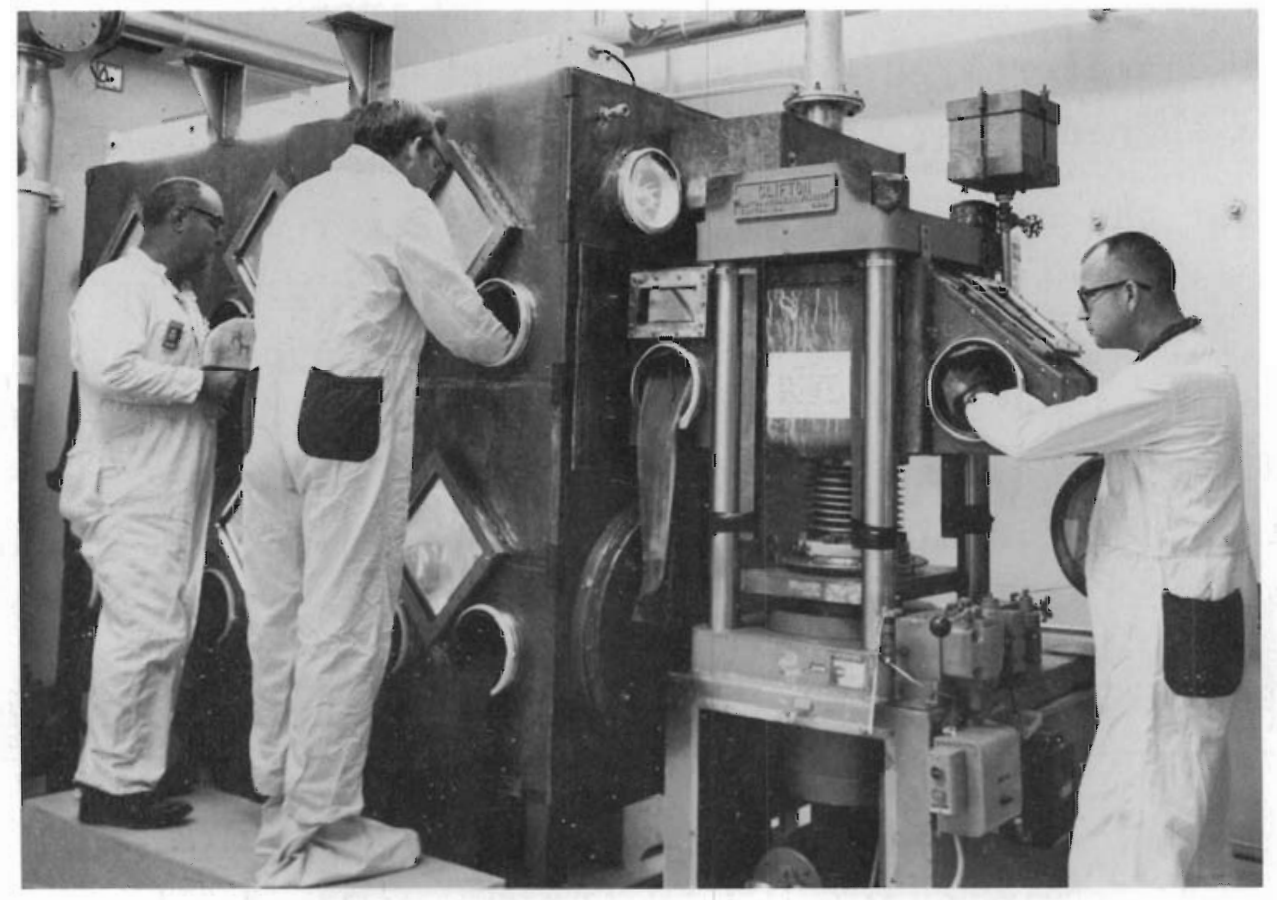

Neg 0672936-1

FIGURE 16. ${ }^{147} \mathrm{Pm}_{2} \mathrm{O}_{3}$ Glove Box with Press Attached

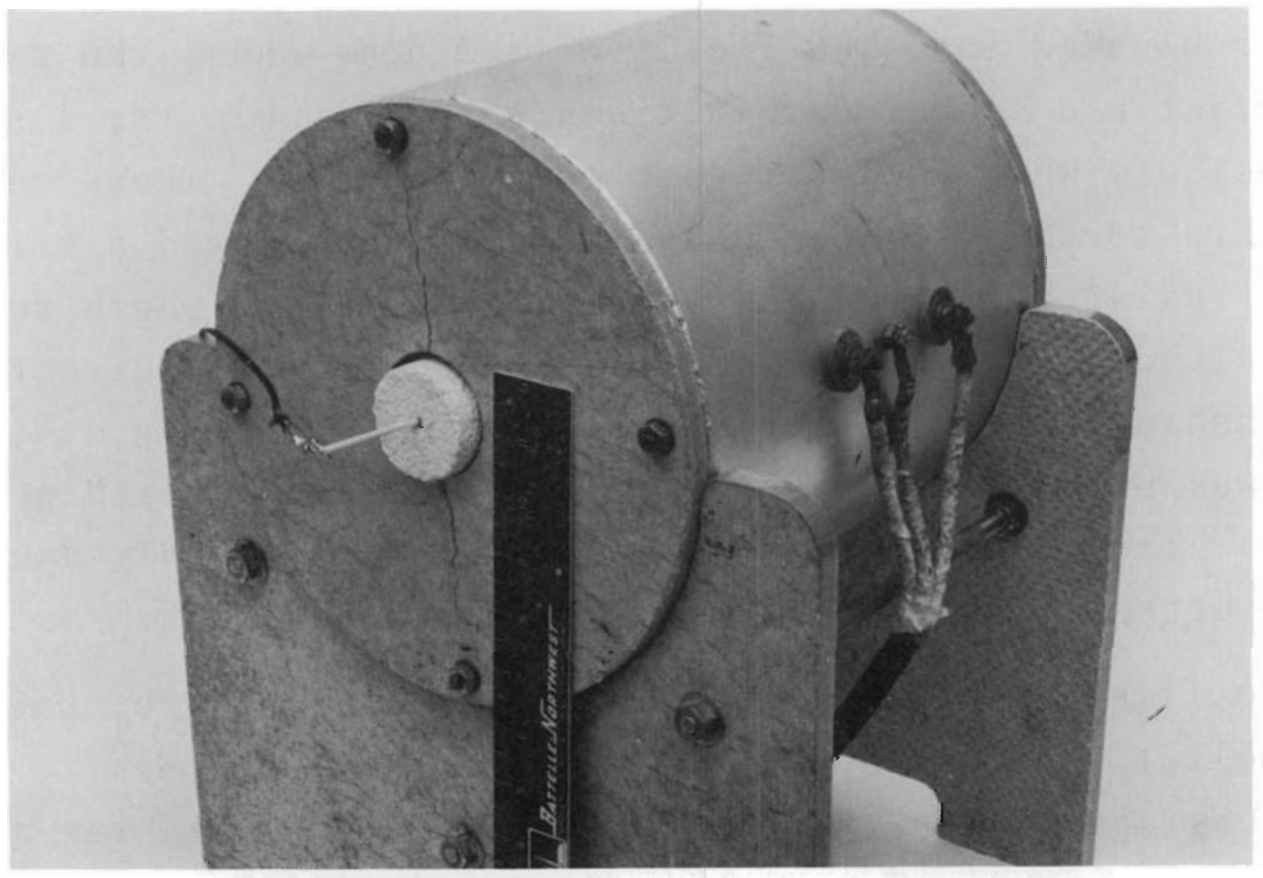

Neg 0672575

$$
\text { FIGURE 17. }{ }^{147} \mathrm{Pm}_{2} \mathrm{O}_{3} \text { Sintering Furnace }
$$


applied. The die sleeve was quickly ejected and an apparently good pellet (Figure 18) was produced. On closer examination, the upper dome was found to be merely resting in position. Removal of the upper dome revealed large concentric and radial cracks in the lower core of the pellet. Upon sintering, these cracks were found more severe rather than to have healed or closed (Figure 19).

The maximum diameter at the break in the pellet was 1.511 in. Several dimensions from 0.020 to 0.030 in. oversized were noted at the broken end of the pellet. Although this end of the pellet was flared due to the internal cracks, the balance of the pellet body was well within the dimensional

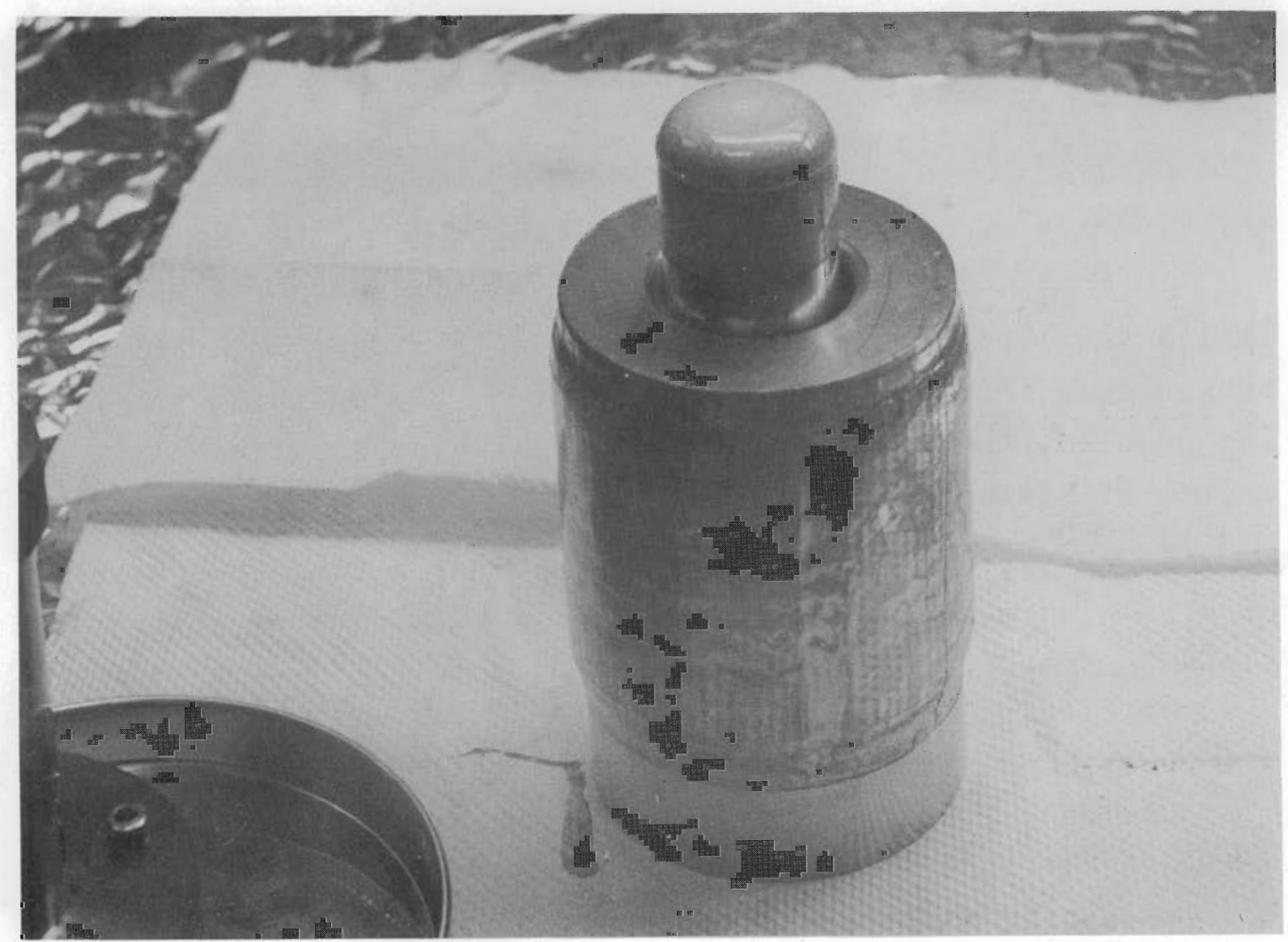

Neg $0672642-5$

FIGURE 18. Promethium Pellet No. 1 as Pressed 


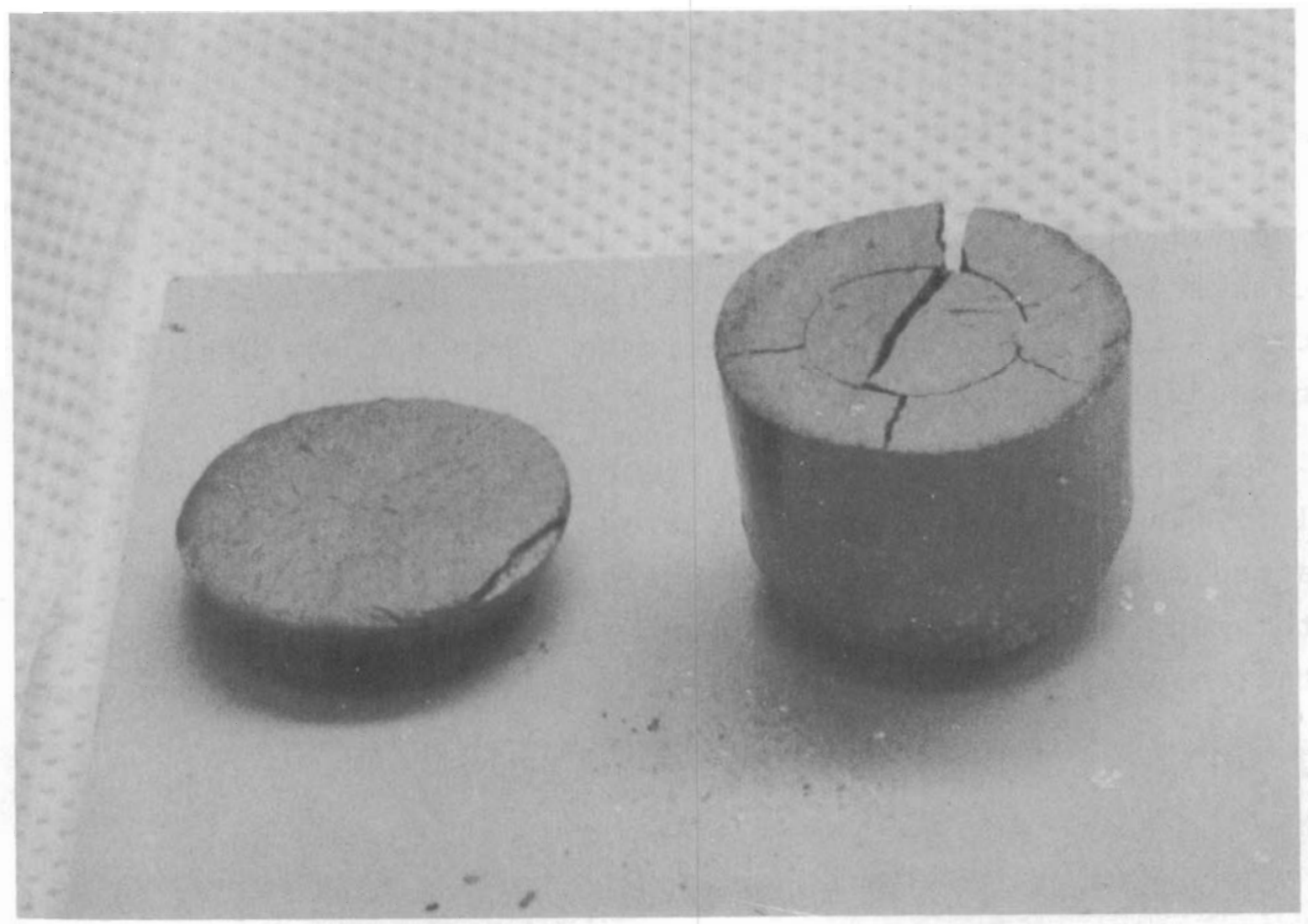

Neg $0672642-1$

FIGURE 19. Promethium Pellet No. 2 as Sintered

limits required for assembly. The length reduction, with the broken dome in place, was satisfactory. Disregarding the cracking problem, the sintering results indicated a satisfactory degree of sintering reduction (Table 7).

At this point in the program, several alternate courses of action were considered to produce a satisfactory pellet for the AMSA heat source. These were:

- Utilize the existing pellet,

- Press and sinter another pellet and modify conditions to hopefully achieve a solid pellet, and

- Use an assembly of wafers to reduce thermal gradients and resultant stresses during processing.

The first two of the preceding courses of action were considered to be the most desirable for initial efforts. 
BNWL -994

TABLE 7. ${ }^{147} \mathrm{Pm}_{2} \mathrm{O}_{3}$ Pellets*

\begin{tabular}{|c|c|c|c|c|c|c|c|c|c|}
\hline \multicolumn{4}{|c|}{ Pressing Data } & \multicolumn{6}{|c|}{ Sintering Data } \\
\hline \multirow{2}{*}{$\begin{array}{l}\text { Pe11et } \\
\text { No. } \\
\end{array}$} & \multirow{2}{*}{$\begin{array}{c}\text { Pressure, } \\
\text { tsi }\end{array}$} & \multirow{2}{*}{\multicolumn{2}{|c|}{$\frac{\text { Dimensions, in. }}{\text { diam }}$}} & \multirow{2}{*}{$\begin{array}{r}\text { Time, } \\
\text { hr }\end{array}$} & \multirow{2}{*}{ Temp., } & \multirow{2}{*}{\multicolumn{2}{|c|}{$\frac{\text { Dimensions, in }}{\text { diam }}$}} & \multirow{2}{*}{\multicolumn{2}{|c|}{$\frac{\text { Reduction, } \frac{:}{0}}{\text { diam } \frac{{ }^{2}}{\text { length }}}$}} \\
\hline & & & & & & & & & \\
\hline PM- 1 & 63 & 1.580 & 1.550 & 30 & 1450 & 1.445 & 1.450 & 8.5 & 6.3 \\
\hline \multirow[t]{2}{*}{ PM- 2} & 63 & 1.580 & 0.900 & 30 & 1450 & 1.485 & 0.860 & 6.0 & 4.4 \\
\hline & & & & 24 & 1500 & \multicolumn{2}{|c|}{$\begin{array}{l}\text { No change on } \\
\text { rerun }\end{array}$} & & \\
\hline PM- 3 & 50 & 1.580 & 1.588 & 30 & 1450 & 1.460 & 1.470 & 7.6 & 7.4 \\
\hline
\end{tabular}

Using a specially modified inner cladding, clamping pressure was applied to the oversize portion of the cracked pellet in an attempt to close the cracks, and thus reduce the pellet sufficiently for assembly. This effort was soon abandoned to prevent high radiation exposure to personnel. It soon became apparent that this pellet could be used only by machining out the inside of the primary cladding. Since this approach would result in a loss of shielding, this assembly (Figure 20) was held in reserve until the results of pressing and sintering a second pellet could be evaluated.

The second attempt to form a monolithic pellet for the AMSA capsule was also unsuccessful. The pellet body did not show cracking but, as with the first pellet, the dome ends broke off. Severe internal cracking in the first pellet was believed to be caused by a buildup in the core temperature after the pellet was pressed due to self-heating of the ${ }^{147} \mathrm{Pm}_{2} \mathrm{O}_{3}$. The core temperature induced greater thermal expansion in the hotter central portion than in the outer surface. This effect was minimized in the second attempt to press the pellet. The die was loaded and only slight pressure (approximately 1000 psi) was applied to consolidate the powder in the fixed form and then released. With the powder fixed in the die in a shape and volume similar to that of the final pressed pellet, it was allowed to stand for $1 \mathrm{hr}$ before applying the final pressure of 63 tsi, so that temperature stabilization 


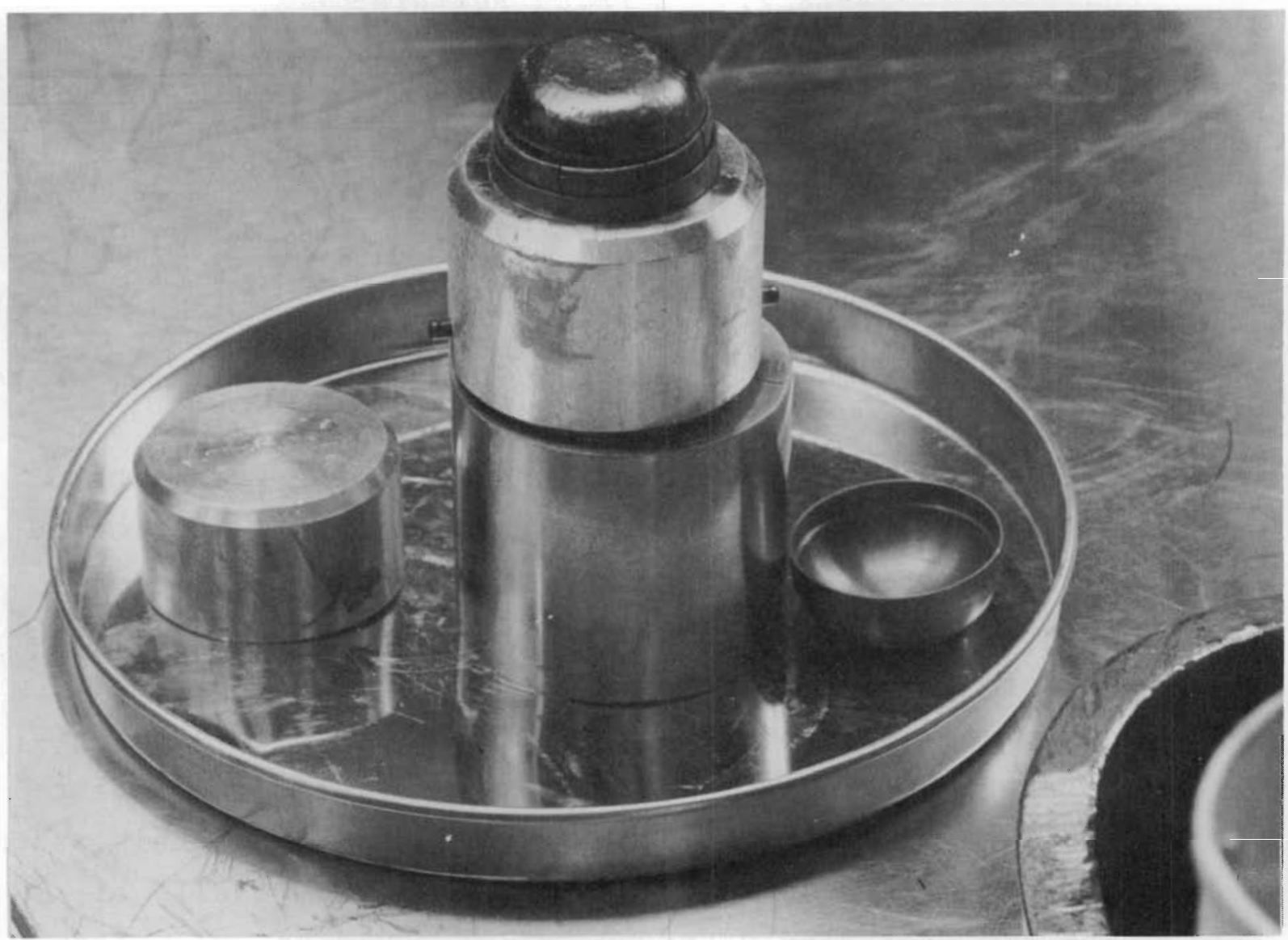

Neg $0673936-2$

FIGURE 20. Promethium Pellet No. 1 Recovery Attempt could occur. Hopefully, after pressing of the pellet was completed and it was ejected from the die, thermal stresses would not build up, since the pellet core was already at a higher temperature than the surface at the time of maximum pressure application. The procedure was demonstrated to be partially effective, as shown by the complete lack of internal cracks in the body of $\mathrm{Pm}_{2} \mathrm{O}_{3}$ Pellet No. 2, (Figure 21). The problem of the broken domes remained. Additional experiments with $\mathrm{Sm}_{2} \mathrm{O}_{3}$, in which three-part die plugs and split ring plugs were used with colloidal graphite die lubricant, demonstrated that sticking of the pellet to the die plug was not the cause of the 
broken domes. A positive answer to this problem has not been determined. It is not a new problem, since earlier pellet pressing with the $\mathrm{Sm}_{2} \mathrm{O}_{3}$ stand-in produced several two- and three-piece pellets, while quite a few of the one-piece assemblies had microcracks. Since these multi-piece $\mathrm{Sm}_{2} \mathrm{O}_{3}$ assemblies did not significantly affect the impact integrity of the capsule (Table 5), this problem was given second priority in producing a fuel core for the AMSA capsule.

The sintering reduction of the second ${ }^{147} \mathrm{Pm}_{2} \mathrm{O}_{3}$ pellet was not nearly as great as the first one. Table 7 shows that even the rerun at $1500{ }^{\circ} \mathrm{C}$ failed to reduce the pellet center section sufficiently for assembly. In a detailed review of

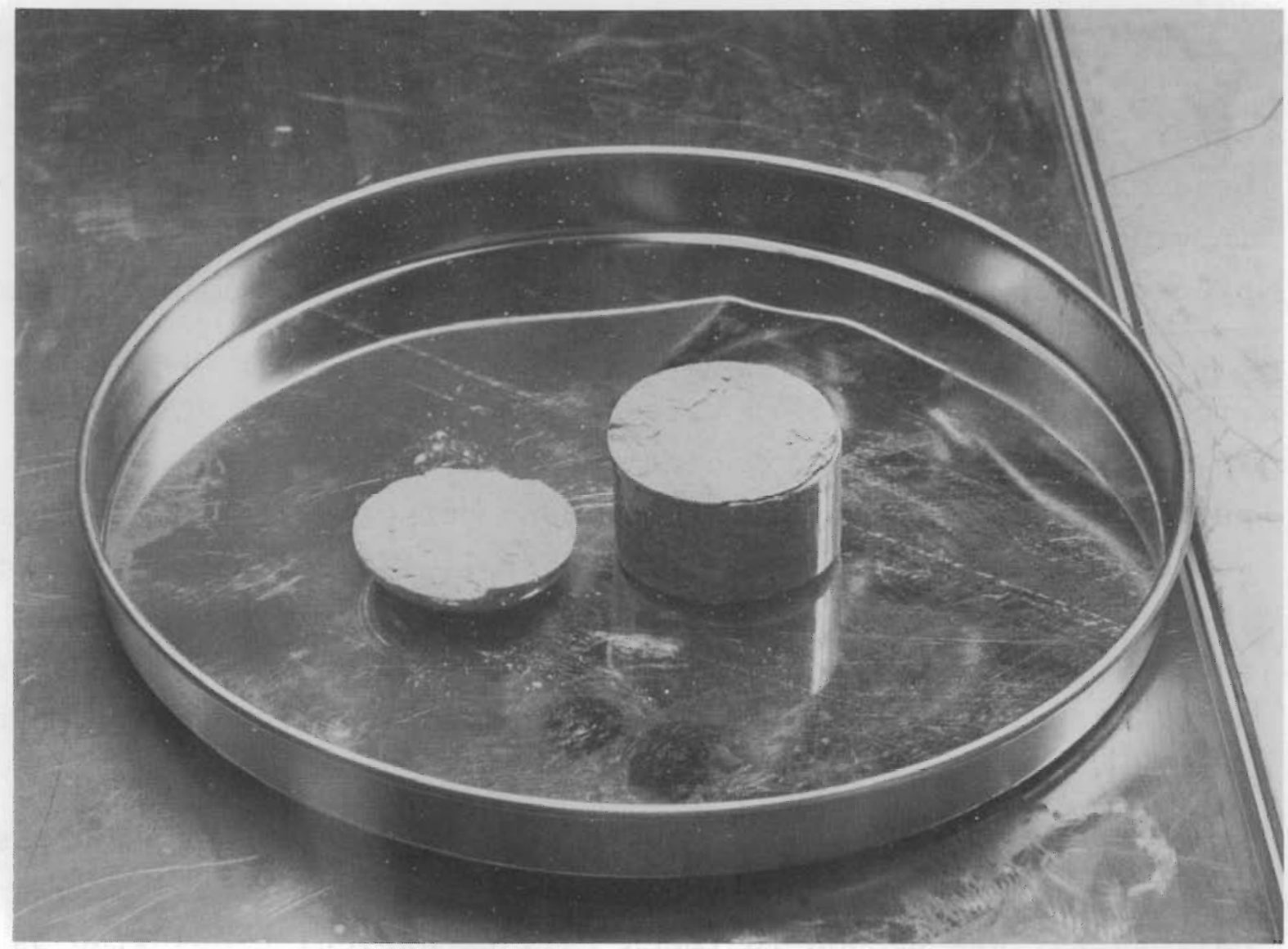

Neg 0673936-3

FIGURE 21. Promethium Pellet No. 2 as Sintered 
prepowder preparation, it was determined that this powder had been prepressed in the "slugging" operation to a density 10 to $15 \%$ higher than that of the first pellet powder. Rather than using applied pressure as a criterion for forming these domeshaped pellets, it became apparent that maintaining a precise length-to-diameter ratio before sintering was of prime importance to produce a pellet for assembly in the specific inner cladding dimensions. The second pellet could be used by sanding approximately $0.020 \mathrm{in}$. off the diameter. This pellet was retained as a possible lower density stand-by, and the first pellet was reprocessed chemically to provide powder for a third pellet.

The loaded die delay procedure developed for use with ${ }^{147} \mathrm{Pm}_{2} \mathrm{O}_{3}$ Pellet 2 was followed in pressing the third and final AMSA pellet. This pellet (Figure 22) was fractured into an upper radiused end cap, a cylindrical body, and a lower radiused end cap. After sintering for $30 \mathrm{hr}$ at $1450{ }^{\circ} \mathrm{C}$ and smoothing the laminarly fractured surfaces by sanding, the three pieces could be assembled into the AMSA inner tantalum cladding.

The weight of the $\mathrm{Pm}_{2} \mathrm{O}_{3}$ loaded in the asembly was $227 \mathrm{~g}$. Process design parameters were based on the use of $235 \mathrm{~g}$ of $\mathrm{Pm}_{2} \mathrm{O}_{3}$, corresponding to a heat output of $65 \mathrm{~W} 30$ days after purification of the promethium. Heat output of the $65 \mathrm{~W}$ source would be reduced to $62.4 \mathrm{~W}$ on October 1, 1967, due to aging of the promethium. A slight weight loss in the three-piece pellet, sustained by chipping of the fractured surfaces, caused a further reduction of $2.1 \mathrm{~W}$ to a final assembled 60.3 wattage.

The proper length-to-diameter ratio of this third and final pellet was assured by using a positive stop during pressing to control the length of the pellet. As Table 7 shows, only 50 tsi was needed to reach this mechanical press stop as compared to the two previous $\mathrm{Pm}_{2} \mathrm{O}_{3}$ pellets, pressed at 63 tsi 


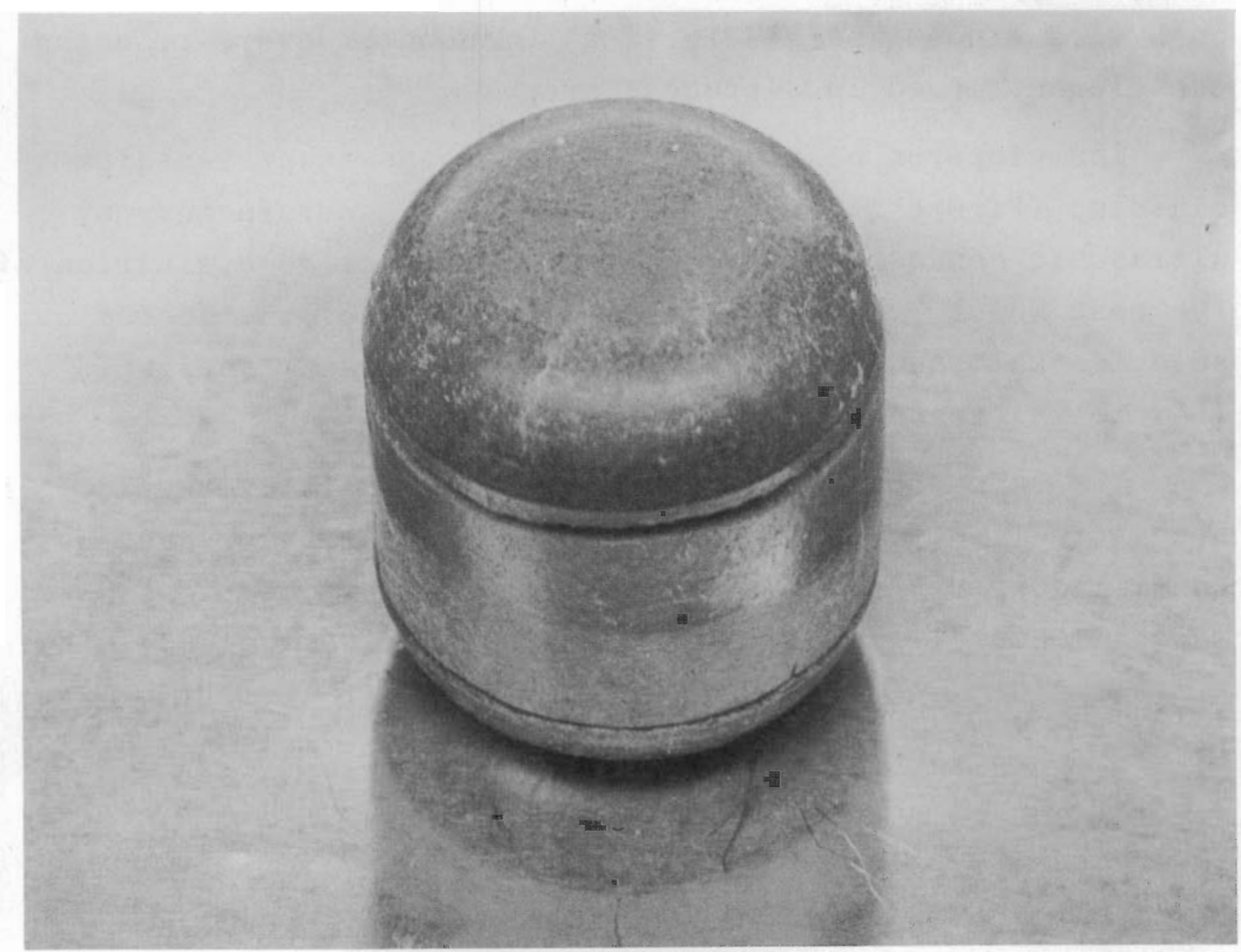

Neg $0673374-1$

\section{FIGURE 22. Promethium Pellet No. 3 as Assembled}

without using a mechanical stop. The final sintered length of this pellet indicates the planned length to be satisfactory for assembly.

\section{ASSEMBLY OF AMSA HEAT SOURCE CAPSULE}

The AMSA process flow and quality control chart (Figure 13) was followed very closely in making the double cladding assemb1y. As expected, only the inner cladding assembly offered significant problems in contamination control. After decontamination was effected and the first weld was made, the capsule remained nonsmearable for the balance of the assembly. This condition confirmed by double smear tests 
using a dry cotton swab and measuring the contamination level on a mica window (beta detection) GM Counter every time the capsule was moved in a process step.

The electron beam weld closure of the inner tantalum cladding (Figure 23) was determined to be satisfactory by ultrasonic nondestructive testing. Destructive evaluation of the test welds, made before and after welding the fueled capsule, established weld penetration for an inner seal weld (Figure 24) to be optimum.

The same procedure was used on the final outer weld. The nondestructive evaluation was noted as satisfactory and was confirmed by destructive examination of the first test weld made immediately before the fueled capsule was welded. The

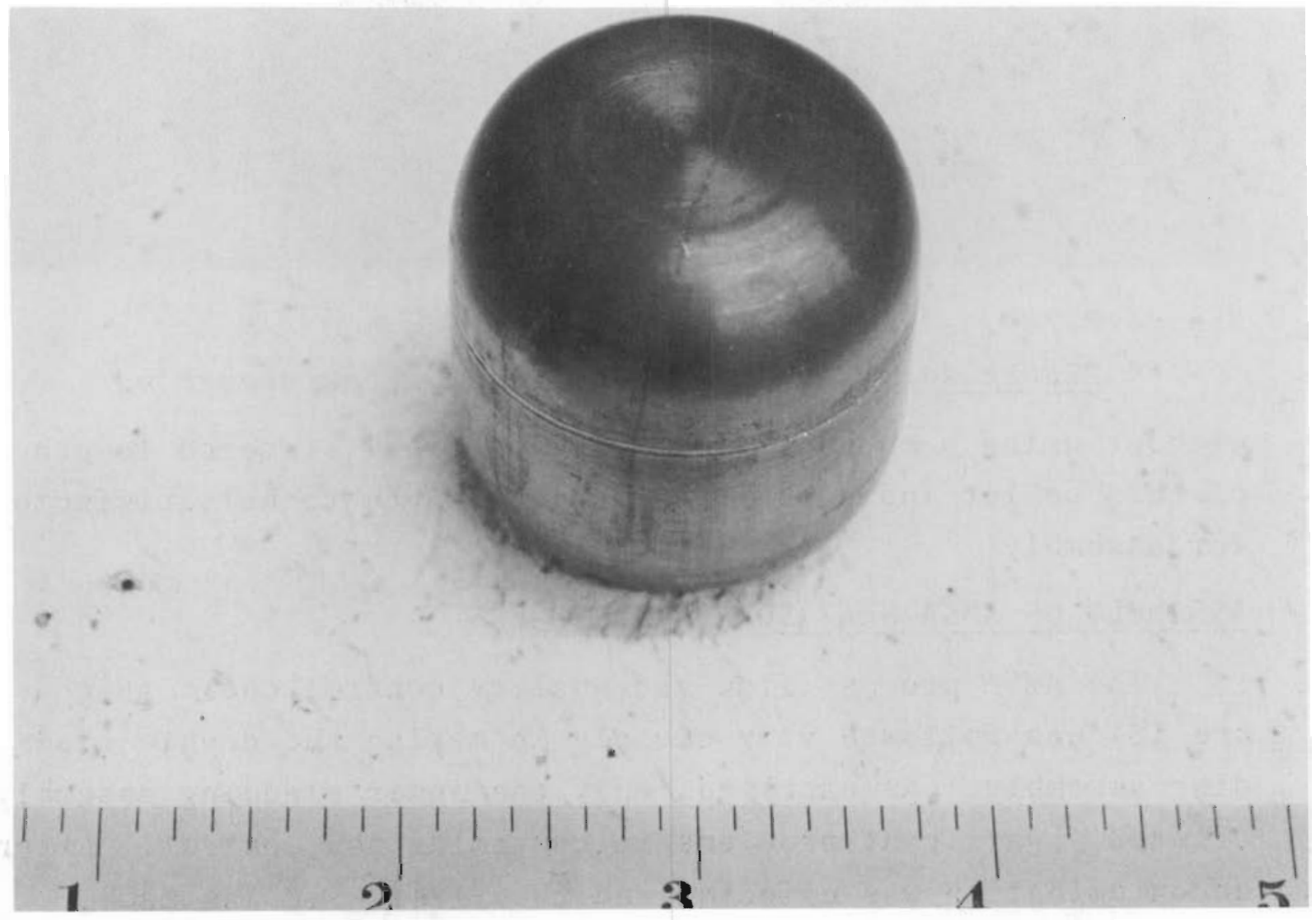

Neg $0673438-3$

EIGURE 23. AMSA Capsule Inner clad Welded 


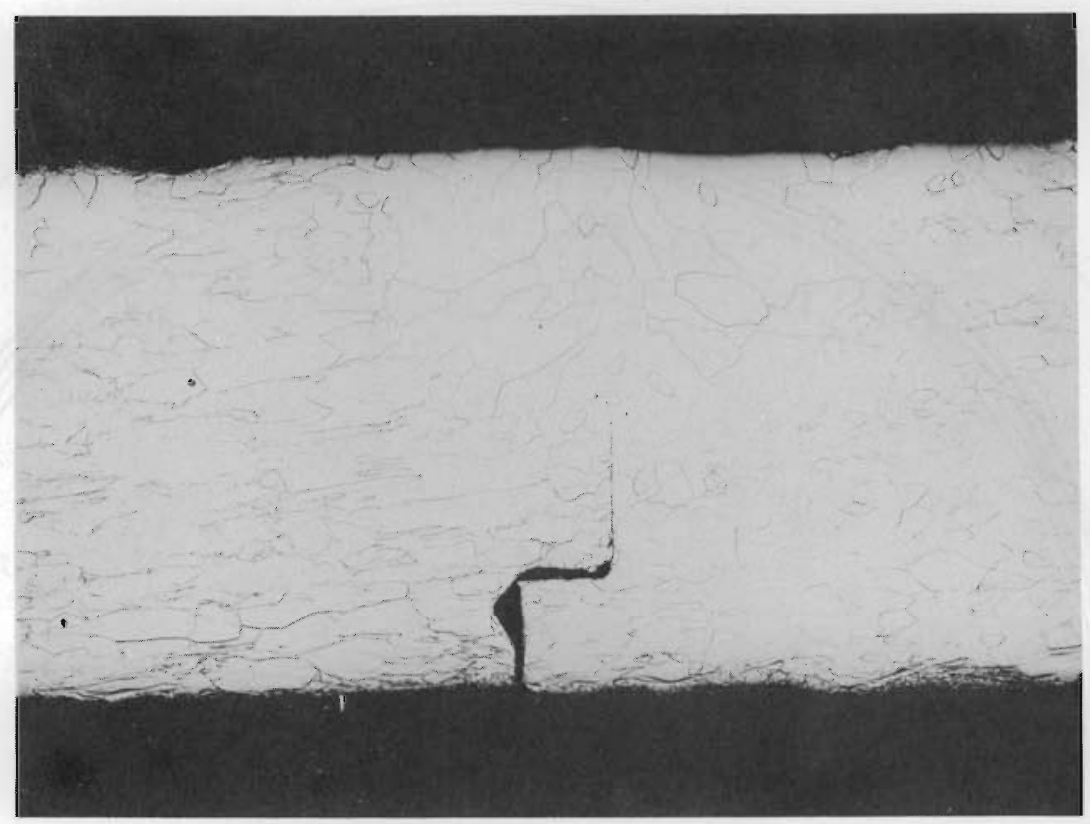

Neg 467-1711

FIGURE 24. Inner Tantalum Weld, Test Capsule

last test weld, made immediately after the fueled capsule was welded, showed penetration to be far less than the desired $85 \%$. A recheck of the fueled capsule (Profile Figure 25) using a dual testing technique was made. Minimum weld penetration of $75 \%$ was noted in some areas. However, most of the circumferential weld exceeded the $85 \%$ desired.

A third test weld using welding parameters identical to those in the previous welds was made to confirm the nondestructive finding. Very slight differences between this weld, the first test weld, and the fueled capsule weld were noted by nondestructive examination of all three welds, and by destructive examination of the test welds. A photomicrograph of the first test weld, considered to be typical of the fueled capsule, is shown in Figure 26.

The promethium fueled capsule was subjected to the quality assurance inspections identified in Figure 13. These quality control inspection tests also are listed in 
BNWL-994

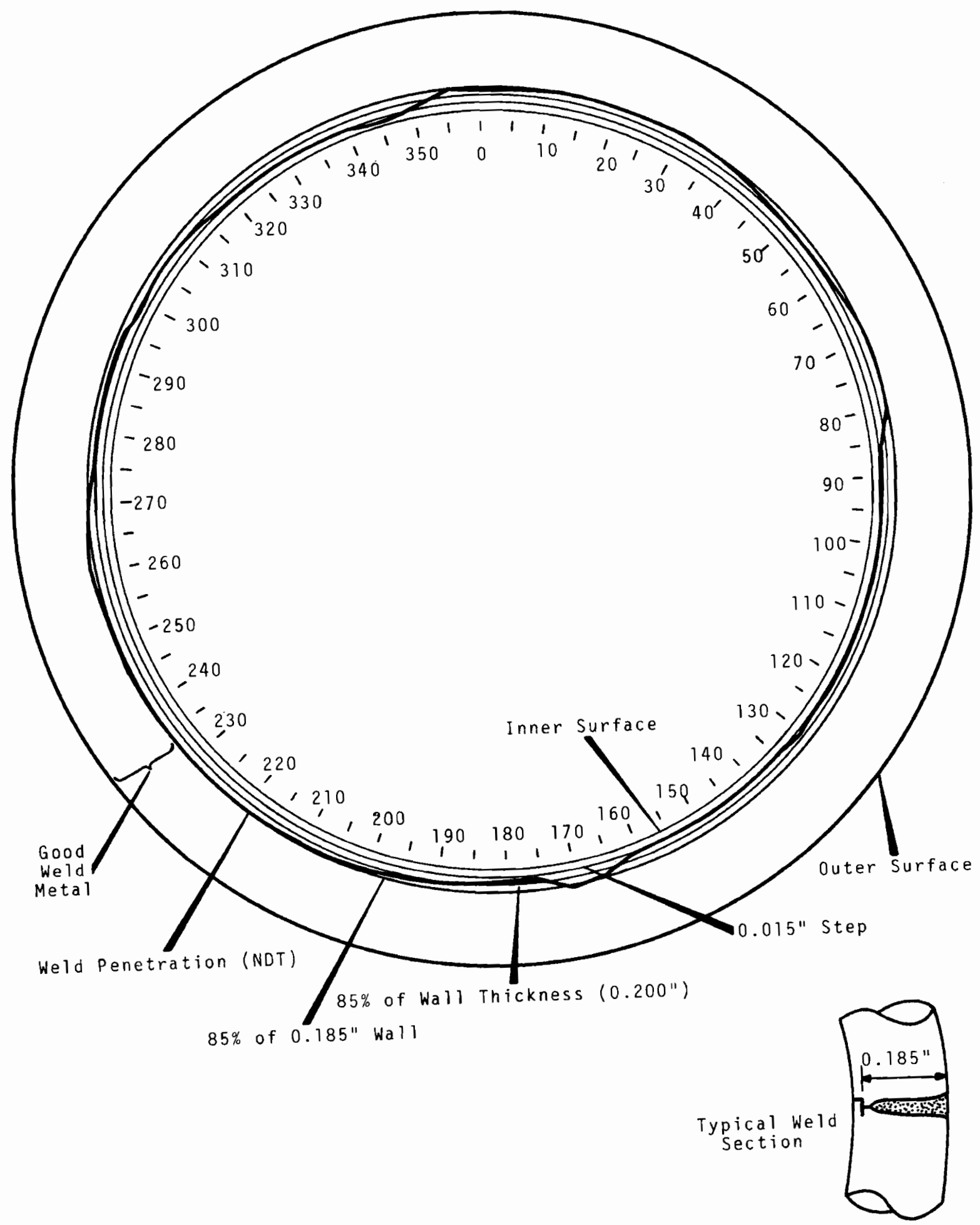

Neg $0681613-3$

FIGURE 25. Nondestructive Test Profize of Fueled Capsule 
BNWL-994

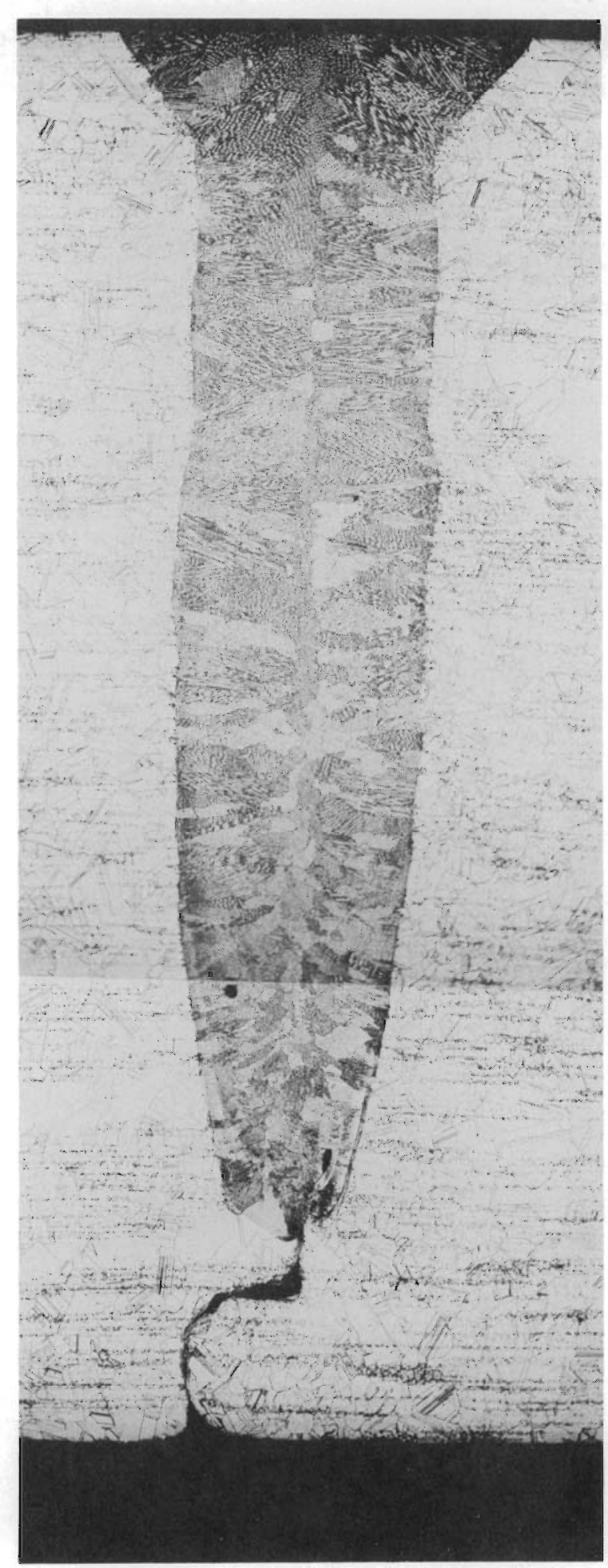

Neg 567-1708 $A$ and $B$

FIGURE 26. Outer L-605 Weld Test Capsule 


\section{BNWL -994}

Appendix Table A-1, and all pertinent test results are accumulated in Tables A-2 through A-5. These tables provide a complete review of the quality control procedures and test results. 
BNWL - 994

\section{HANDL ING AND SHIPPING}

Handling procedures for the AMSA ${ }^{147} \mathrm{Pm}_{2} \mathrm{O}_{3}$ heat source capsule were recommended by Battelle-Northwest personne1. These procedures covered the detailed packaging required for the shipment of the fueled capsule, as well as recommended steps for optimum safety in removing the capsule from the shipping container, placement into and removal from the thermal preconditioner unit (TPU), and storage of the heat source capsule. A11 required handling tools were supplied with the capsule, and general radiological safety procedures used at Pacific Northwest Laboratory were reviewed.

Final photographs of the completed AMSA capsule are shown as Figure 27, and the radiation map of measured dosage is shown as Figure 28. The ICC approved specification - 2R container is shown with the special copper chill block holding fixture in Figure 29. This assembly, when loaded into the shipping barre1 (Figure 30), was checked for detailed temperature and radiation measurements (Figures 31 and 32 ).

The AMSA promethium-147 capsule was delivered to the Air Force at Wright-Patterson Air Force Base in March 1968. The capsule, a 60 thermal watt heat source as of October 1, 1967, was held in storage until delivery of the Inertial Measuring Unit. A reduction to approximately $54 \mathrm{~W}$ occurred during this storage period. Flight and laboratory tests using the capsule were performed by the Air Force, and the capsule was returned to Battelle-Northwest in April, 1968. 


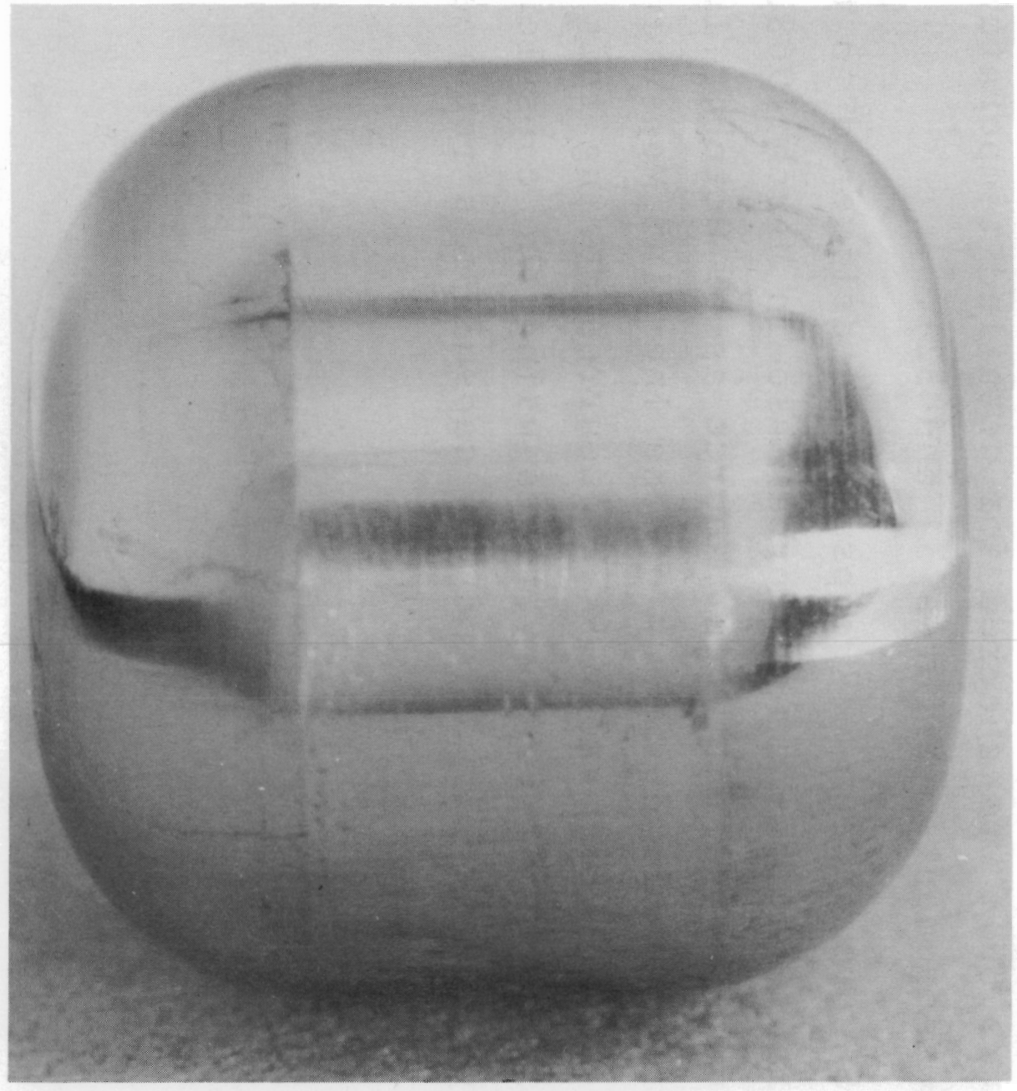

Neg $0673446-1$

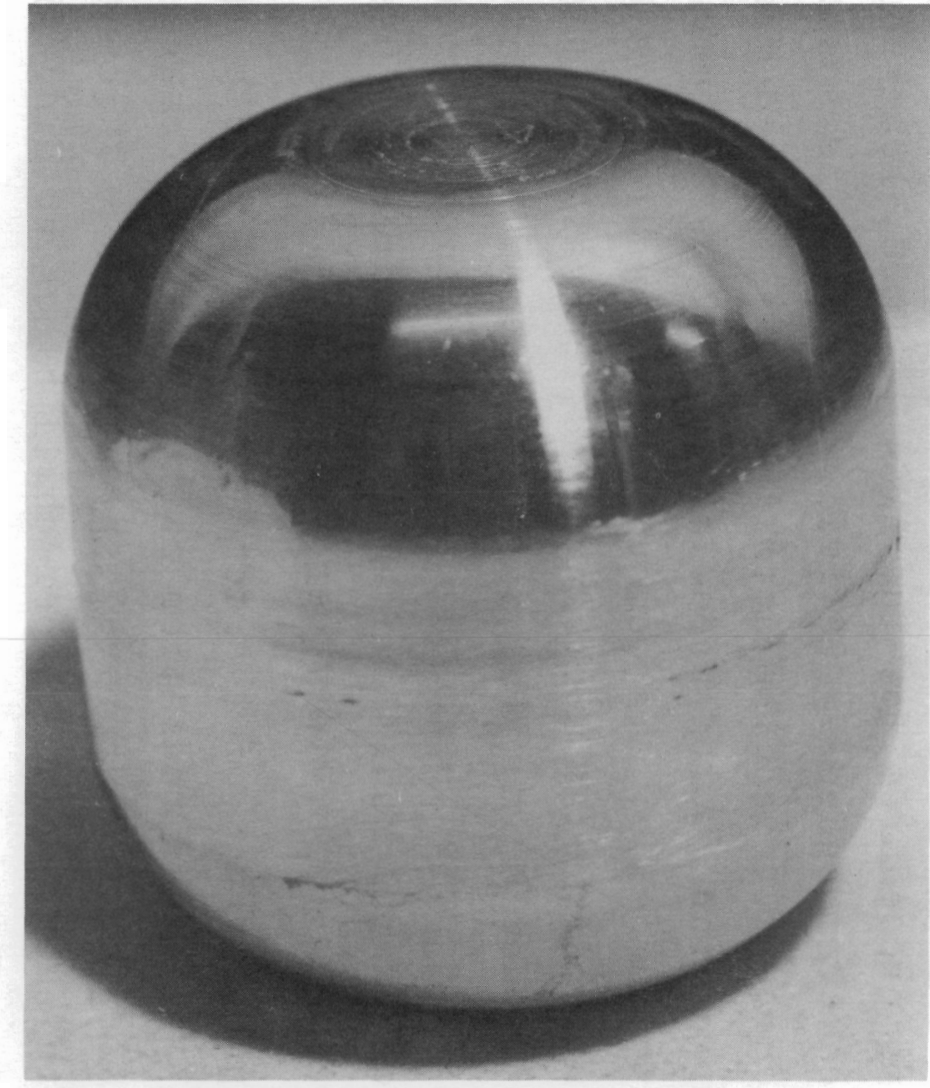

Neg 0673446-2

FIGURE 27. Two Views of Final AMSA Fueled Capsule 

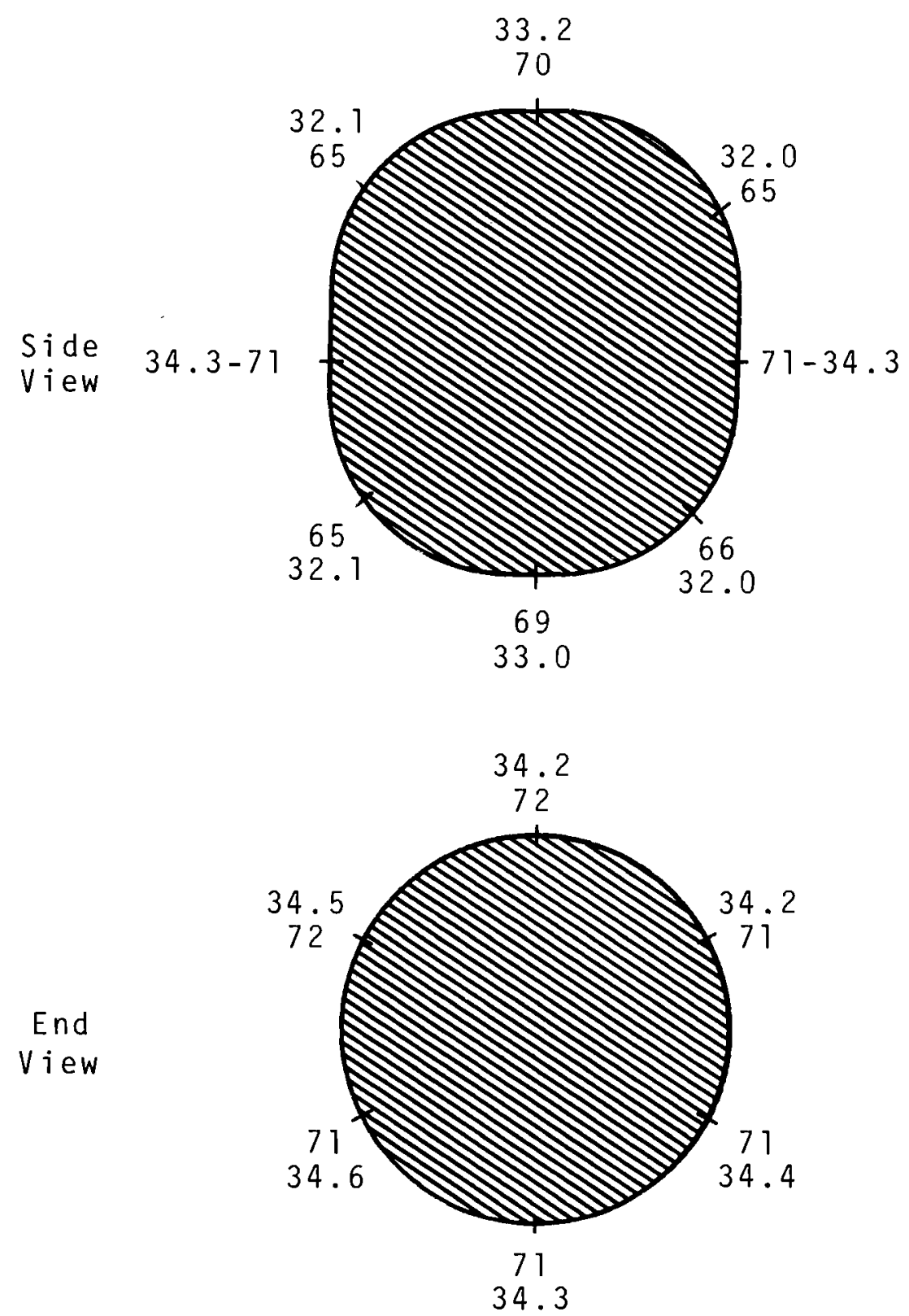

Inner Numbers Are $12 \mathrm{in.}$ from Center of Capsule, in mr/hr Outer Numbers Are $18 \mathrm{in.}$. from Center of Capsule

Neg 0681613-4

FIGURE 28. Radiation Mapping 


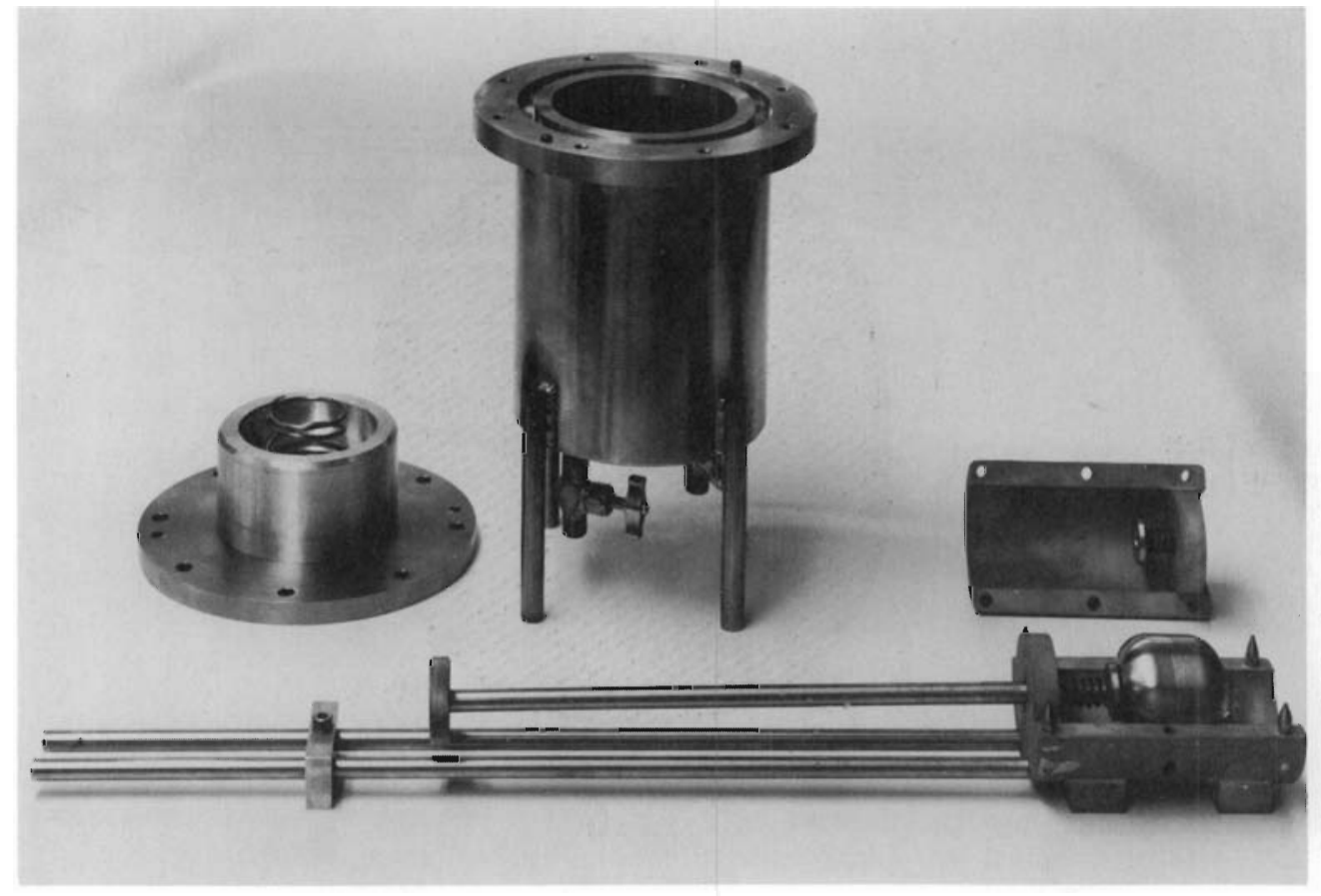

Neg $0673109-1$

FIGURE 29. 2R ICC Specified Container with Holding Fixture

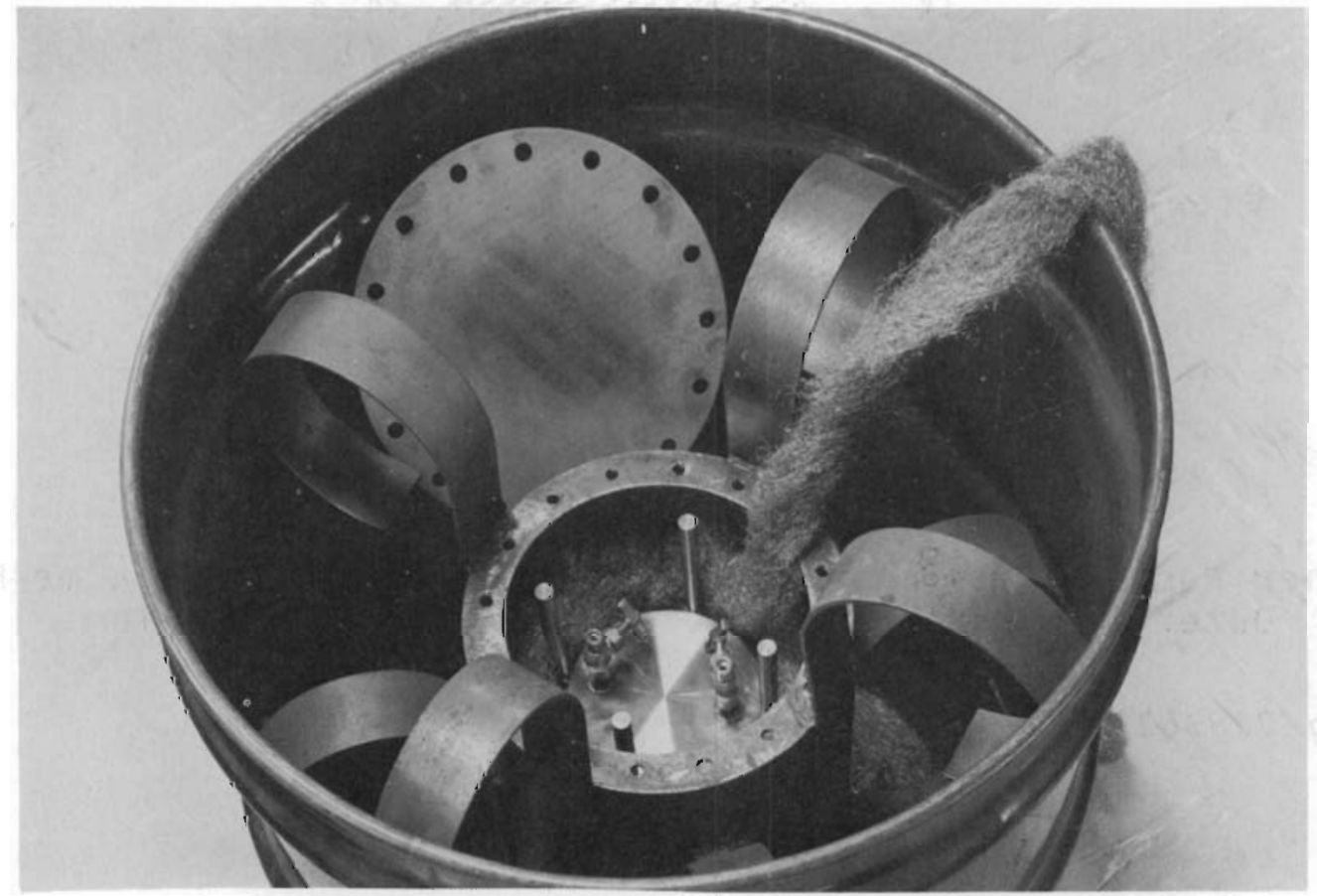

Neg $0673826-2$

FIGURE 30. Shipping Barrel 
All Readings with Barrel Closed, or Taken Immediately After Standing at Room Temperature for 64 Hours.

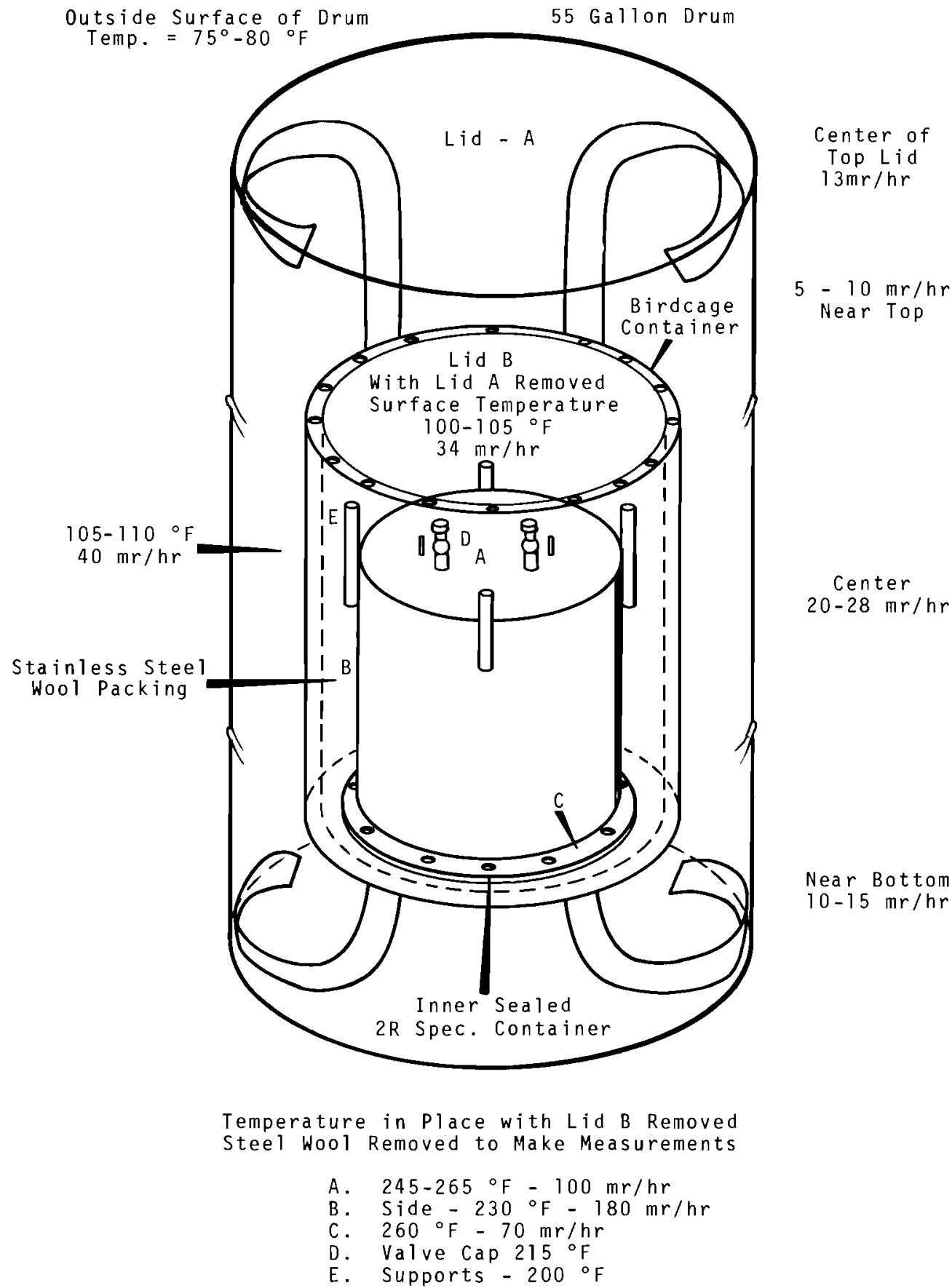

Neg $0681613-2$

FIGURE 31. Radiation and Temperature Profize of Shipping Container 
2R Inner Container (Sealed) Removed from Shipping Drum after 64 Hours. Allowed to Stand 30 Min. in Air - Room Temp. - Sitting on Table Top (Non-Metallic)
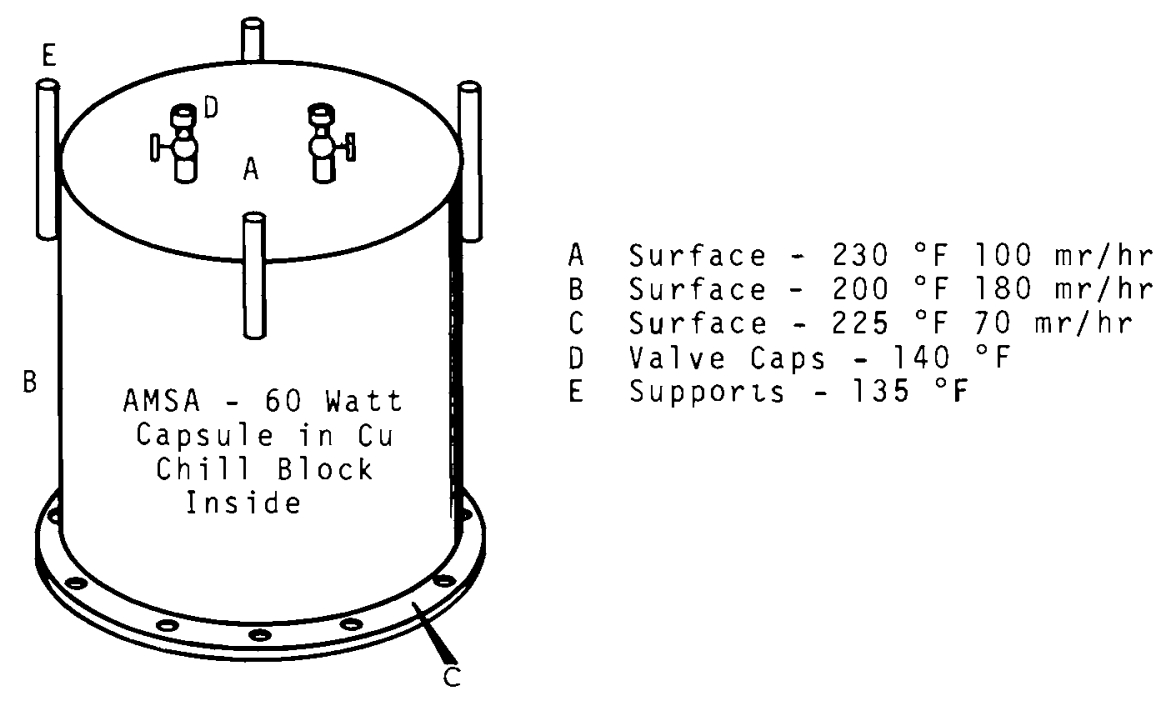

After 2R Spec. Container Opened, Immediately after Above Measurements were Made.

NOTES:

1. Temp. Top of Copper Block $245^{\circ} \mathrm{F}$ Side of Copper Block $245^{\circ} \mathrm{F}$

2. Radiation Field to Hands in Screwing Eye Bolt into Top of Copper Block $=220 \mathrm{mr} / \mathrm{hr}$

3. Temp. of AMSA Capsule Immediately Af ter Removing Copper $\mathrm{Chill} \mathrm{Block}-330^{\circ} \mathrm{F}$

4. Temp. of AMSA Capsule after Sitting on Ceramic Surface for two Hours in Still Room Temperature Air-480 ${ }^{\circ} \mathrm{F}$

A Ring Gage 2.005" diam. Passed over Capsule at this Point with Minimum Interference - Length $=1.999^{\prime \prime}-$ wt. -745.2 grams

5. Radiation $=1100 \mathrm{mr} / \mathrm{hr}$ with CP Beta Shield in Actual Contact with Capsule. (Uncorrected for Geometry of CP Chamber)

Neg $0681613-1$

FIGURE 32. Radiation and Temperature Profize of $2 R$ Container 


\section{REFERENCES}

1.W. J. Dalby. 9312 AMSA Fuel Capsule Impact Tests, SC-DR-67-718. Sandia Corporation, Albuquerque, New Mexico, August 1967.

2. L. A. Bray and H. H. Van Tuyz. Laboratory Development of a Carrier-Precipitation Process for the Recovery of Strontium from Purex Wastes, $H W-69534$. Available from Clearinghouse for Federal Scientific and Technical Information, Springfield, Virginia, May 9, 1961.

3. W. V. DeMier, G. L. Richardson, A. M. Platt, and W. H. Swift. Development of Radioactive Waste Fractionization and Packaging Technology, $H W-S A-2786$. Available from Clearinghouse for Federal scientific and Technical Information, Springfield, Virginia, January 1963.

4. L. A. Bray. Denitration of Purex Wastes with Sugar, HW-76973 Rev. Available from Clearinghouse for Federal Scientific and Technical Information, Springfield, Virginia, Apriz 1963.

5. E. J Wheelwright, F. P. Roberts, U. L. Upson, L. J. Kirby, T. R. Myers, and H. H. Van Tuyz. IonExchange Separation of Kilocurie Quantities of $\overline{H i g h}$ Purity Promethium, BNWL-318. Pacific Northwest Laboratory, Richland, Washington, December 1966. 


\section{ACKNOWLEDGEMENTS}

The authors are indebted to the many co-workers at the Pacific Northwest Laboratory who performed much of the work reported on this document. Personal acknowledgements, identified with areas of major performance, are noted below. Special thanks are extended to the many contributors.

- R. J. Baker and C. E. Vogel

- R. F. Boolen and D. E. Dewitt

- W. F. Riemath, G. O. Hammer, and E. H. Shade

- R. H. Beauchamp

- R. W. Steffen and F. M. Coffman

- E. J Wheelwright, F. P. Roberts and $U$. L. Upson
Program Management Welding Process Development Pressing, Sintering, and Assembly

Metallography

Nondestructive

Testing

Chemica1 Development, Radiation Mapping, and Calorimetry

Special recognition is extended, additionally, to

W. J. Dalby, Sandia Laboratory, for his cooperation and effort in planning and conducting the many impact tests. 
APPENDIX 


\section{TABLE A-1. Types of Quality Assurance Inspections*}

1. Dimension Measurements 8. X-ray Diffraction

2. Dye Penetrant

3. Impurity Analys is

4. Density Determination

5. He Leak Check

6. Metallography

7. Isotopic Analysis
9. Radiography

10. U1trasonic Testing

11. Decontamination Smear Check

12. Radiation Mapping

13. Gamma Spectrometry

14. Calorimetry

* See Figure 13

TABLEA-2. Fueled Capsule Component Test Results

\begin{tabular}{|c|c|c|c|}
\hline \multirow[b]{2}{*}{1} & \multirow[b]{2}{*}{$\begin{array}{l}\text { Dimension } \\
\text { Measurements }\end{array}$} & Inner Clad & Outer Clad \\
\hline & & $\begin{array}{l}1.598 \times 1.592 \text { in. } \\
\text { overa11 }\end{array}$ & $\begin{array}{l}1.999 \times 1.997 \mathrm{in} . \\
\text { overa11 }\end{array}$ \\
\hline & & $\frac{0.0605 \text { in. }}{0.0615 \text { in. }}$ wa 11 & $\frac{0.199 \text { in. }}{0.202 \text { in. }}$ wa11 \\
\hline 2 . & $\begin{array}{l}\text { Dye } \\
\text { Penetrant* }\end{array}$ & $\begin{array}{l}\text { No relevant } \\
\text { indications }\end{array}$ & No relevant indications \\
\hline 3 . & $\begin{array}{l}\text { Impurity } \\
\text { Analysis }\end{array}$ & $\begin{array}{l}\text { Certificate of } \\
\text { Analysis Page A-2 }\end{array}$ & $\begin{array}{l}\text { Test Report } \\
\text { Page } A-3\end{array}$ \\
\hline * & $\begin{array}{l}\text { Dye Penetrant - } \\
\text { consists of (1) } \\
\text { (2) emulsifying } \\
\text { developing for }\end{array}$ & $\begin{array}{l}\text { ZY Glo-Post emulst } \\
\text { soaking in penetro } \\
4 \text { min, and (3) was } \\
\text { zack light read-or }\end{array}$ & $\begin{array}{l}\text { ier technique } \\
\text { t } 20 \mathrm{~min} \text {, } \\
\text { ing dry and }\end{array}$ \\
\hline
\end{tabular}




\section{FANSTEEL CERTIFICATION}

CERTIFICATE OF ANALYSIS

Customer

Battelle Nomorial Inst1tute

Material

Tantalum Dod 1.600

Production No. 63h-032688-0

Lot No. 69:2053

\section{CHEMICAL ANALYSIS}

Quantity $15.551 \mathrm{IbB}$.
Date October 20, 1967

Purchase Order No. B8S-TT7

\section{FANSTEEL}

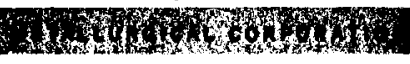

NUMBER ONE TANTALUM PLACE NORTH CHICAGO, ILLINOIS • 60064 Phone: $312 / 689-4900 \quad$ TWX: 312/244.4530

$12^{n}+1 / 8^{\prime \prime}-0.000^{\prime \prime}$

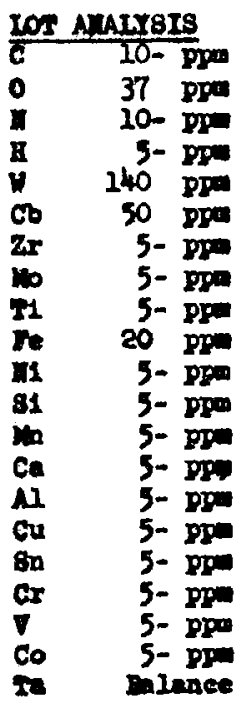

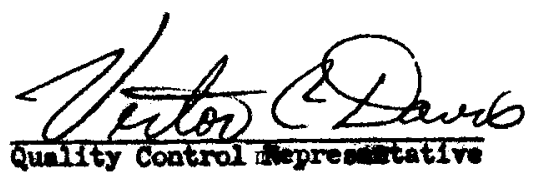

Gunitty control apresictative

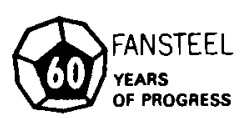




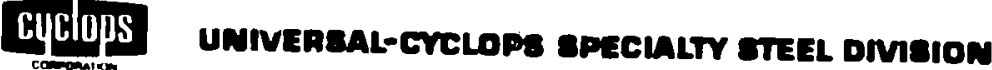

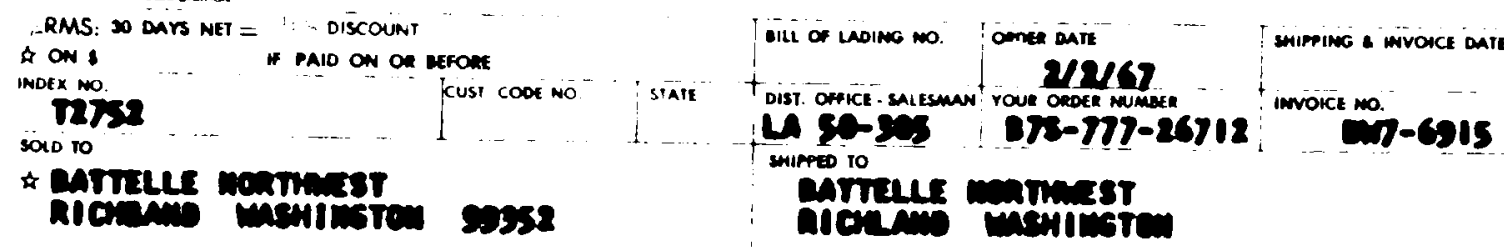

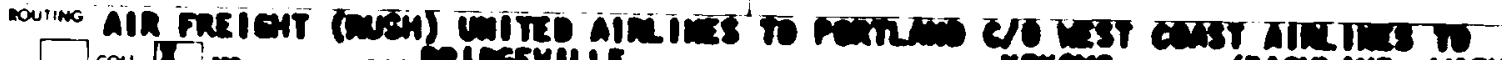 PACKing a marking instructionis ros. Colcesvice r/c Intring Inicinan men provar mis

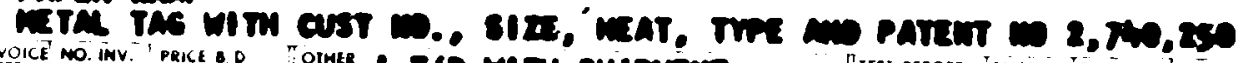

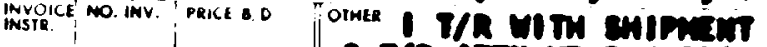

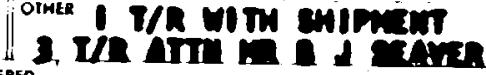 \\ STOCK CONTRO MO OUAN. ORDERED SIIE AND DESCRIPTHON

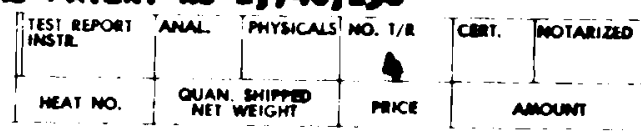

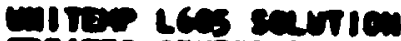

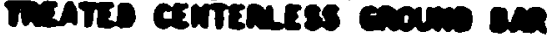

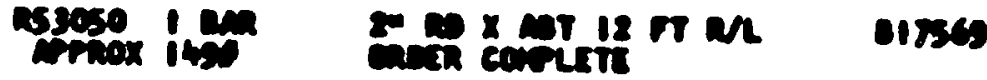

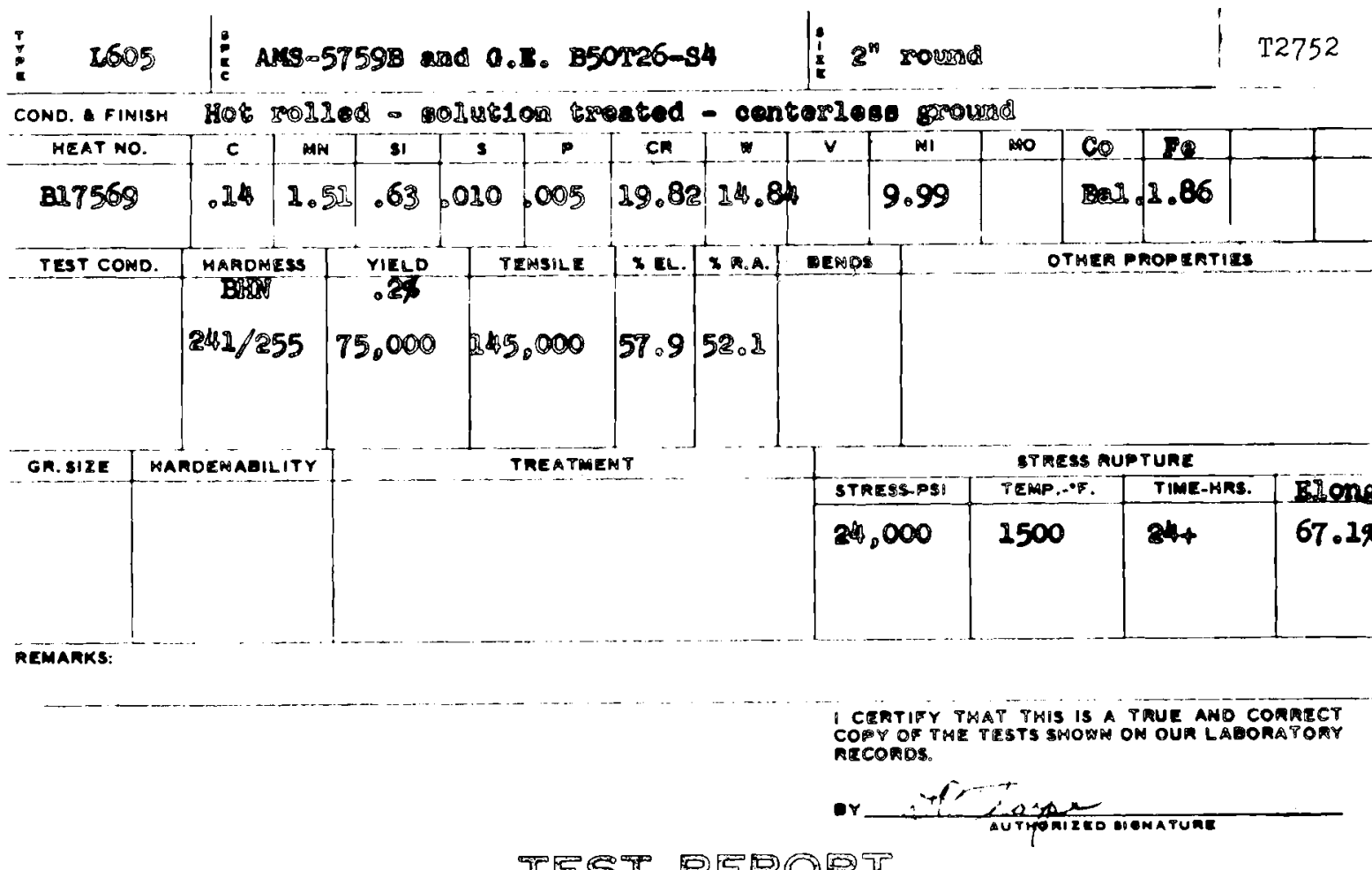

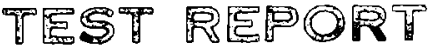


TABLEA-3. Fueled Capsule Pellet Test Results

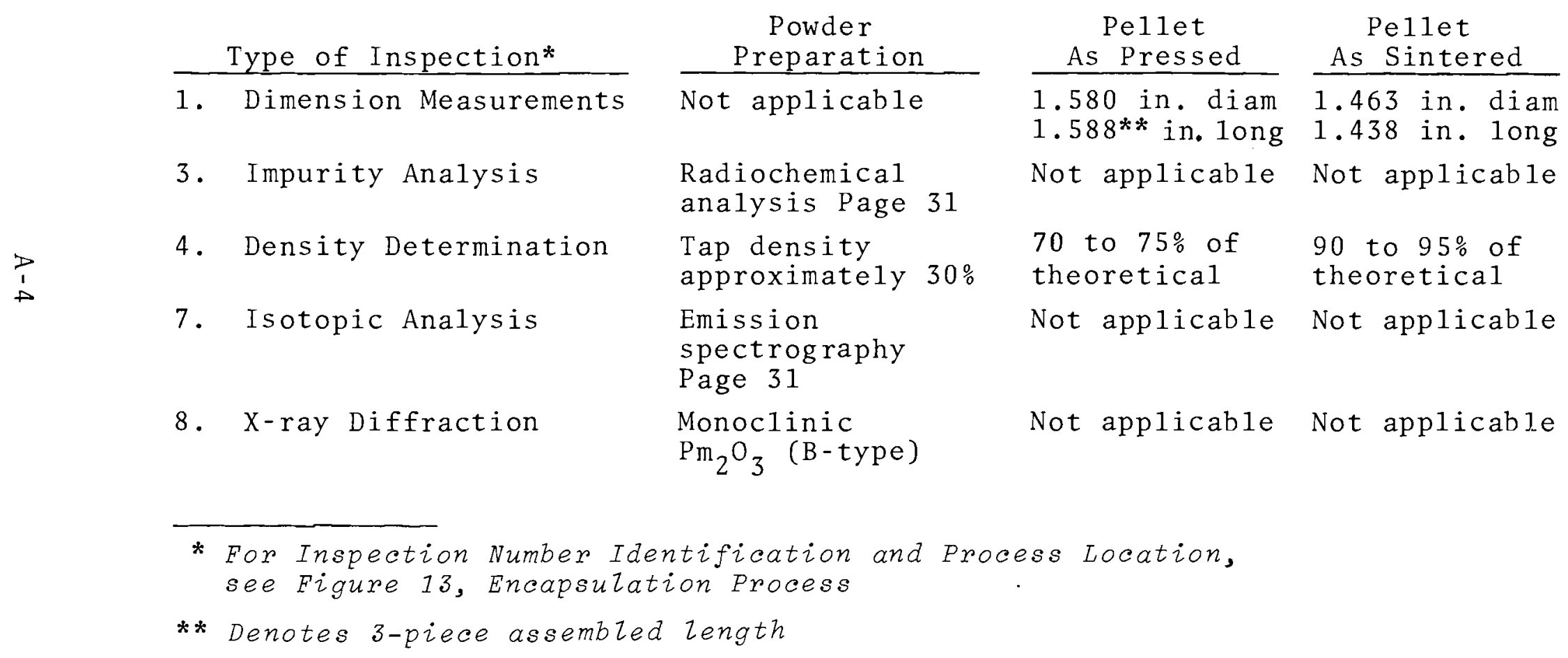


TABLE A-4. Fueled Capsule Assembly Test Results

Type of Inspection

5. He Leak*1 Check

6. Metallography

10. U1trasonic*2

11. Decontamination Smear Check*3

12. Radiation*4 Mapping
Inner Capsule

Assemb1y

Background

Test weld

Figure 24

Minimum weld

$50 \%$ or greater

Nonsmearable

$1 \mathrm{~m}$

$7.4 \mathrm{mr} / \mathrm{hr}$
Outer Clad

Assemb 1y

Background

Test weld

Figure 26

73 to $93 \%$ weld profile

Figure 25

Nonsmearable

$1 \mathrm{~m}$

6.8 to $7.2 \mathrm{mr} / \mathrm{hr}$

*1 CEC Helium Leak Detector

1. Chamber with capsule evacuated to 0.05 Torr $(30 \mathrm{~min})$

2. Backfizled with He to 25 psi $(5 \mathrm{~min})$

3. Sensitivity check before and after capsule leak test 4. Minimum detectable leak--less than $7 \times 10^{-7} \mathrm{std}$.
$\mathrm{cm}^{2} / \mathrm{sec}$

*2 Broadband Ultrasonic Tester - 15 MHz lithium sulphate; spherically focused transducers used to establish normal incidence and shear wave test techniques.

*3 Contamination Smear Test--mica window G-M calibrated by beta-gamma source.

*4 CP Type ION Chamber--calibrated by NBS standards 
TABLE A-5. AMSA Fueled Capsule Final Test Results Type of Inspection

1. Dimension Measurements

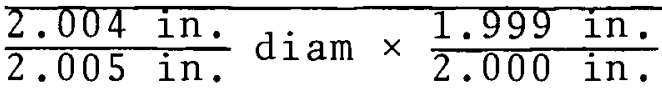
length*1

5. He Leak Check No indication either before or after final we1d bead removal

9. Radiography*2

Both inner and outer cladding revealed areas of incomplete we ld penetration-previously noted on ultrasonic test

10. U1trasonic Test In addition to previous ultrasonic test, side walls were inspected for laminar type flaws with normal incidence test. No defect found.

11. Decontamination Smear Check Nonsmearable both before and after final weld bead removal

12. Radiation Mapping $\frac{6.8 \mathrm{mr} / \mathrm{hr}}{7.2 \mathrm{mr} / \mathrm{hr}}$ at $1 \mathrm{~m}$. Profile as shipped. Page 53

13. Gamma Spectrometry See gamma energy plot Page A-7 14. Calorimetry $60.3 \pm 0.2 \mathrm{~W} 10 / 1 / 67$ See additional information Page A-8

* 1 This dimensional test was made after capsule was placed on ceramic brick for a period of $2 \mathrm{hr}$ and temperature was recorded at $480^{\circ} \mathrm{F}$. Ring gage, (Go gage) $2.005 \mathrm{in}$. ID, furnished by AC Electronics per sketch SK-51222, passed over the capsule when fitted cold to the "hot" capsule.

*2 Radiographic Source: Iridium 192 Isotope Radiographic Film. Kodak Type "M" Source-Film Distance: 20 in. Exposure Time: 13 min. Eight exposures were made (A through $H$ ) in $22-1 / 2^{\circ}$ increments. One exposure was made through end of capsule. 
BNWL - 944

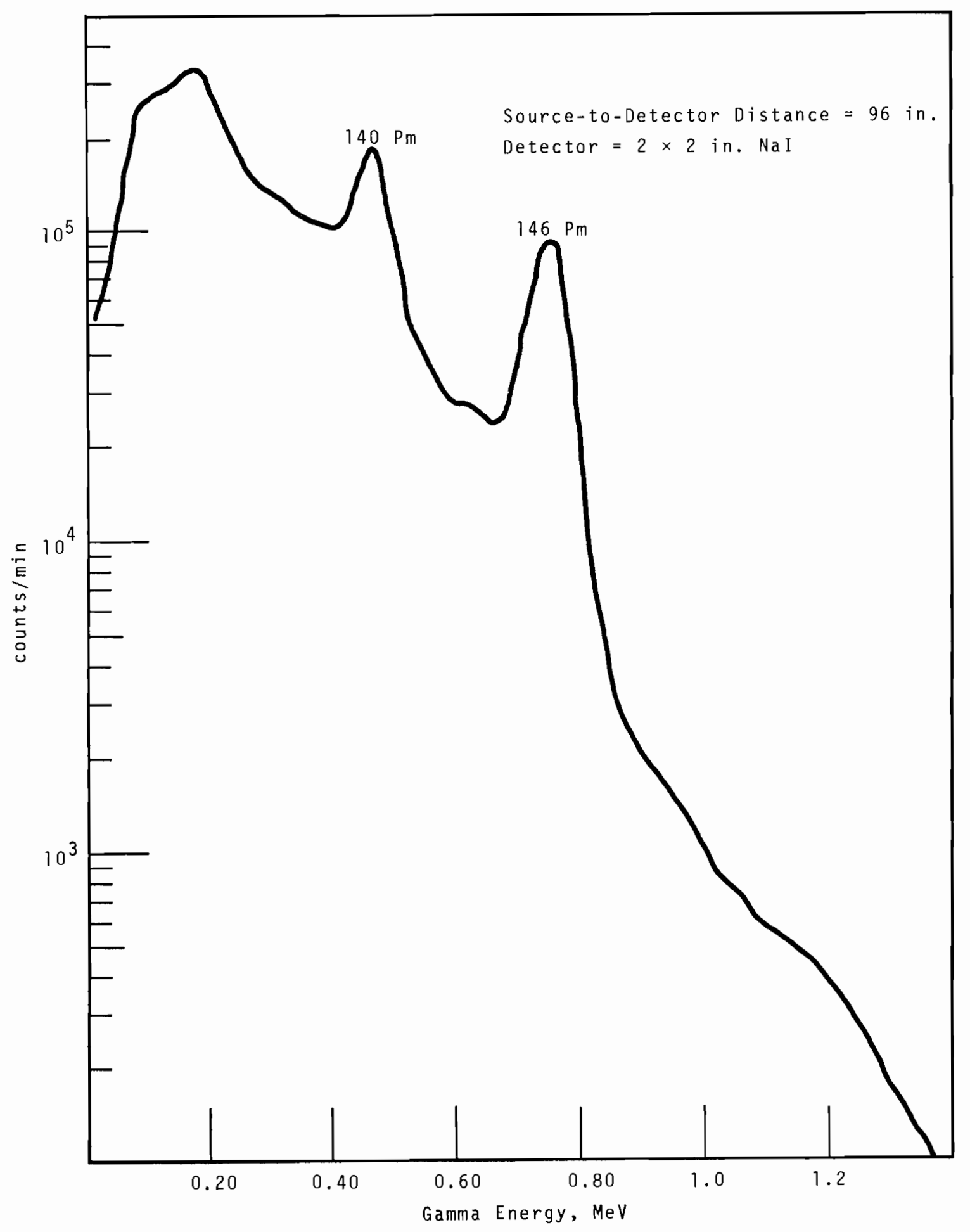

FIGURE A-1. Gamma Spectrum of $60 \mathrm{~W},{ }^{147} \mathrm{Pm}_{2} \mathrm{O}_{3}$ AMSA Capsuze 
BNWL -994

\section{TABLE A-6. AMSA Capsule CaZorimeter Data}

Calorimeter Factor (for conditions of this test): $1.0825 \mathrm{~W} / \mathrm{mV}$ $( \pm 0.10 \%$ at $95 \%$ confidence)

Decay Factor: $\lambda=1 \mathrm{n} 1 / 2 /(957 \times 24)=3.01788 \times 10^{-5} \mathrm{hr}^{-1}$

\begin{tabular}{|c|c|c|c|c|c|c|}
\hline Date & Hour & $\begin{array}{c}\text { Time, } \\
\mathrm{hr}\end{array}$ & $\begin{array}{c}\text { Measured } E^{*} \\
\mathrm{mV}\end{array}$ & $\begin{array}{c}\text { Ca1culated } \\
\mathrm{mV}\end{array}$ & $E_{O} * *$ & $\begin{array}{c}E_{0}-\overline{E^{\prime}} \\
\end{array}$ \\
\hline \multirow[t]{2}{*}{$10 / 6$} & 1200 & 0 & 55.524 & 55.524 & & +2 \\
\hline & $\begin{array}{l}1400 \\
1600 \\
1800 \\
2000 \\
2200 \\
2400\end{array}$ & $\begin{array}{r}2 \\
4 \\
6 \\
8 \\
10 \\
12\end{array}$ & $\begin{array}{l}55.525 \\
55.523 \\
55.510 \\
55.508 \\
55.506 \\
55.498\end{array}$ & $\begin{array}{l}55.528 \\
55.530 \\
55.520 \\
55.521 \\
55.523 \\
55.518\end{array}$ & & $\begin{array}{l}+6 \\
+8 \\
-2 \\
-1 \\
+1 \\
-4\end{array}$ \\
\hline \multirow[t]{2}{*}{$10 / 7$} & $\begin{array}{l}0200 \\
0400 \\
0600 \\
0800 \\
1000 \\
1200\end{array}$ & $\begin{array}{l}14 \\
16 \\
18 \\
20 \\
22 \\
24\end{array}$ & $\begin{array}{l}55.495 \\
55.490 \\
55.485 \\
55.488 \\
55.484 \\
55.480\end{array}$ & $\begin{array}{l}55.518 \\
55.517 \\
55.515 \\
55.522 \\
55.521 \\
55.520\end{array}$ & & $\begin{array}{r}-4 \\
-5 \\
-7 \\
0 \\
-1 \\
-2\end{array}$ \\
\hline & $\begin{array}{l}1400 \\
1600 \\
1800 \\
2000 \\
2200 \\
2400\end{array}$ & $\begin{array}{l}26 \\
28 \\
30 \\
32 \\
34 \\
36\end{array}$ & $\begin{array}{l}55.490 \\
55.485 \\
55.488 \\
55.478 \\
55.470 \\
55.452\end{array}$ & $\begin{array}{l}55.534 \\
55.532 \\
55.538 \\
55.531 \\
55.527 \\
55.512\end{array}$ & & $\begin{array}{r}+12 \\
+10 \\
+16 \\
+9 \\
+5 \\
-10\end{array}$ \\
\hline \multirow[t]{3}{*}{$10 / 8$} & $\begin{array}{l}0200 \\
0400 \\
0600 \\
0800 \\
1000 \\
1200\end{array}$ & $\begin{array}{l}38 \\
40 \\
42 \\
44 \\
46 \\
48\end{array}$ & $\begin{array}{l}55.465 \\
55.445 \\
55.435 \\
55.435 \\
55.445 \\
55.455\end{array}$ & $\begin{array}{r}55.528 \\
55.511 \\
55.505 \\
55.508 \\
55.522 \\
55.535 \\
\end{array}$ & & $\begin{array}{r}+6 \\
-11 \\
-17 \\
-14 \\
0 \\
+13 \\
\end{array}$ \\
\hline & & & & {$[\Sigma]=13.060$} & {$[\Sigma]$} & $=+14$ \\
\hline & & & & $\begin{array}{r}\bar{E}_{\mathrm{o}}=55.522 \\
{\left[\Sigma\left(\mathrm{E}_{\mathrm{o}}-\right.\right.}\end{array}$ & $\begin{array}{l}m V \text { e } \\
\left.\left.\bar{E}_{0}\right)^{2}\right]\end{array}$ & $\begin{array}{l}=+0.6 \\
=1738\end{array}$ \\
\hline
\end{tabular}

Standard deviation of mean $(\sigma)= \pm 0.0085 \mathrm{mV}= \pm 0.015 \%$

$95 \%$ Confidence error $= \pm 0.03 \mathrm{mV}= \pm 0.06 \%$

Capsule power at 1200 October $6\left(\mathrm{t}_{\mathrm{o}}\right)=1.0825(55.522)$

Capsule power at 1200 October $1=60.10 \mathrm{e}^{120 \frac{ \pm}{\lambda}}=60.072 \pm 0.07$

* Two-hour period average, taken from chart record. * Measured average, corrected for decay $\left(E_{0}=E e^{\lambda t}\right)$. 


\section{DISTRIBUTION}

No. of

Copies

\section{OFFSITE}

1

AC Electronics

Division of General Motors Route 128

Wakefield, Massachusetts 01881

W. W. Burroughs, Jr.

1

AEC Chicago Patent Group

G. H. Lee

AEC Division of Space Nuclear Systems

G. P. Dix

$\underline{\text { AEC Division of Technical Information Extension }}$

AEC Library, Washington

J. N. Maddox

R. W. Shivers

Air Force Aero Propulsion Laboratory

Mr. Lintz

Capt. D. C. DePree

Maj.J. S. Pizzuto

1

Andrews Air Force Base

Washington, D.C. 20331

$\operatorname{Maj} \cdot$. . R. Crane

$1 \quad$ Kirtland Air Force Base

Albuquerque, N.M.

Capt. B. E. Watlington

1

Sandia Corporation, Albuquerque (AEC)

V. E. Bl ake

\section{ONSI TE-HANFORD}

1

AEC Chicago Patent Group

R. K. Sharp (Richland) 
No. of

Copies

1

3

66
AEC Richland Operation Office

C. L. Robinson

Batte11e Memoria1 Institute

Batte 11 e-Northwest

F. W. Albaugh

R. J. Baker

A. L. Bement

E. A. Berreth

R. F. Boolen

D. W. Brite

T. D. Chika1la

G. M. Dalen

N. C. Davis (10)

D. R. de Halas

K. Drumhe11er

E. A. Evans

P. L. Farnsworth

H. T. Fullam

R. L. Gibby

S. Goldsmith

W. L. Hampson

J. E. Hansen

J. H. Jarrett

J. N. Judy

R. L . Knecht

C. E. Leach

D. C. Lehfeldt

L. G. Merker

R. L. Moore

M. B. Ne1son

R. E. Nightingale

A. M. Platt

F. P. Roberts

R. K. Robinson

C. A. Rohrmann

C. E. Saari

R. C. Smith

R. W. Steffen

K. R. Sump

U. L. Upson

H. H. Van Tuy 1

C. E. Voge 1

E. E. Voiland

R. G. Wheeler

E. J Wheelwright

Technical Information Files

Technical Publications

Distr -2 